\title{
First detection of acceleration and deceleration in protostellar jets?
}

\section{Time variability in the Chamaeleontis II outflows ${ }^{\star}, \star \star$}

\author{
A. Caratti o Garatti ${ }^{1,2}$, J. Eislöffel ${ }^{1}$, D. Froebrich ${ }^{3}$, B. Nisini ${ }^{4}$, T. Giannini ${ }^{4}$, and L. Calzoletti ${ }^{4}$ \\ 1 Thüringer Landessternwarte Tautenburg, Sternwarte 5, 07778 Tautenburg, Germany \\ e-mail: [caratti;jochen]@tls-tautenburg.de \\ 2 Dublin Institute for Advanced Studies, 31 Fitzwilliam Place, Dublin 2, Ireland \\ e-mail: alessio@cp.dias.ie \\ 3 Centre for Astrophysics and Planetary Science, University of Kent, Canterbury, CT2 7NH, UK \\ e-mail: df@star.kent.ac.uk \\ 4 INAF - Osservatorio Astronomico di Roma, via Frascati 33, 00040 Monte Porzio, Italy \\ e-mail: [nisini;giannini;calzoletti]@oa-roma.inaf.it
}

Received 15 January 2009 / Accepted 27 April 2009

\begin{abstract}
Context. Kinematical and time variability studies of protostellar jets are fundamental for understanding the dynamics and the physics of these objects. Such studies remain very sporadic, since they require long baselines before they can be accomplished.

Aims. We present for the first time a multi-epoch (20 years baseline) kinematical investigation of HH 52, 53, and 54 at optical and near-IR wavelengths, along with medium (optical) and high resolution (NIR) spectroscopic analyses, probing the kinematical and physical time variability conditions of the gas along the flows.

Methods. By means of multi-epoch and multi-wavelength narrow-band images, we derived proper motions (PMs), tangential velocities, velocity and flux variability of the knots. Radial velocities and physical parameters of the gas were derived from spectroscopy. Finally, spatial velocities and inclination of the flows were obtained by combining both imaging and spectroscopy.

Results. The PM analysis reveals three distinct, partially overlapping outflows. Spatial velocities of the knots vary from $50 \mathrm{~km} \mathrm{~s} \mathrm{~s}^{-1}$ to $120 \mathrm{~km} \mathrm{~s}^{-1}$. The inclinations of the three flows are $58 \pm 3^{\circ}, 84 \pm 2^{\circ}$, and $67 \pm 3^{\circ}$ (HH 52, HH 53, and HH 54 flows, respectively). In 20 years, about $60 \%$ of the observed knots show some degree of flux variability. Our set of observations apparently indicates acceleration and deceleration in a variety of knots along the jets. For about $20 \%$ of the knots, mostly coincident with working surfaces or interacting knots along the flows, a relevant variability in both flux and velocity is observed. We argue that both variabilities are related and that all or part of the kinetic energy lost by the interacting knots is successively radiated. The physical parameters derived from the diagnostics are quite homogeneous along and among the three outflows. The analysis indicates the presence of very light $\left(N_{\mathrm{H}} \sim 10^{3} \mathrm{~cm}^{-3}\right)$, ionised $\left(X_{\mathrm{e}} \sim 0.2-0.6\right)$, and hot $\left(T_{\mathrm{e}} \sim 14000-26000 \mathrm{~K}\right)$ flows, impacting a denser medium. Several knots are deflected, especially in the HH 52 flow. At least for a couple of them (HH 54 G and G0), the deflection originates from the collision of the two. For the more massive parts of the flow, the deflection is likely the result of the flow collision with a dense cloud or with clumps. Finally, we discuss the possible driving sources of the flows.
\end{abstract}

Key words. stars: pre-main sequence - ISM: jets and outflows - ISM: Herbig-Haro objects - ISM: kinematics and dynamics

\section{Introduction}

Protostellar jets and outflows represent fundamental processes in star formation. These outflows are a direct consequence of the accretion mechanism in young stellar objects (YSOs) during their earliest phase (see e.g. Cabrit et al. 1990; Shu et al. 2000; Reipurth \& Bally 2001), when a fraction of the infalling material is ejected in the form of collimated jets. The interaction between the ejecta and the circumstellar medium occurs via radiative shocks.

In the very young and still deeply embedded sources, these flows are mostly observed in molecular $\left(\mathrm{H}_{2}\right)$ and atomic

\footnotetext{
* Based on observations collected at the European Southern Observatory (Paranal and La Silla, Chile) (55.C-0908, 62.I0136(A), 63.I-0031(A), 69.C-0269(A), 74.C-0235(A), 77.C-0047(B), 078.C-0089(A))

$\star \star$ Appendices A-E are only available in electronic form at http://www . aanda.org
}

([Fe II]) lines in the near-infrared (NIR) (see e.g. Schwartz et al. 1987; Eislöffel et al. 1994a; Davis et al. 1994). At later phases Class I and Class II - the sources also become visible in the optical, and their jets can then be traced by optical lines as [S II] $(6716,6730 \AA$ ) and $\mathrm{H} \alpha$, as well (see e.g. Graham \& Elias 1983; Mundt 1998b). Observations of the emission lines from the shock-heated gas allow us to study physical parameters in detail, such as the density, the temperature, the ionisation fraction, and the abundances (see e.g. Hartigan et al. 1994; Bacciotti \& Eislöffel 1999; Lavalley-Fouquet et al. 2000). Moreover, since shocks in outflows trace ejecta that are progressively older with distance from the source, it is also possible to gain important information about their driving source properties, reconstructing the YSO mass ejection history.

A description of the gas dynamics, however, only becomes available through measurements of the flow velocity. Spectroscopic radial velocity measurements are necessary, but not sufficient for deriving the flow speed, since they deliver only 


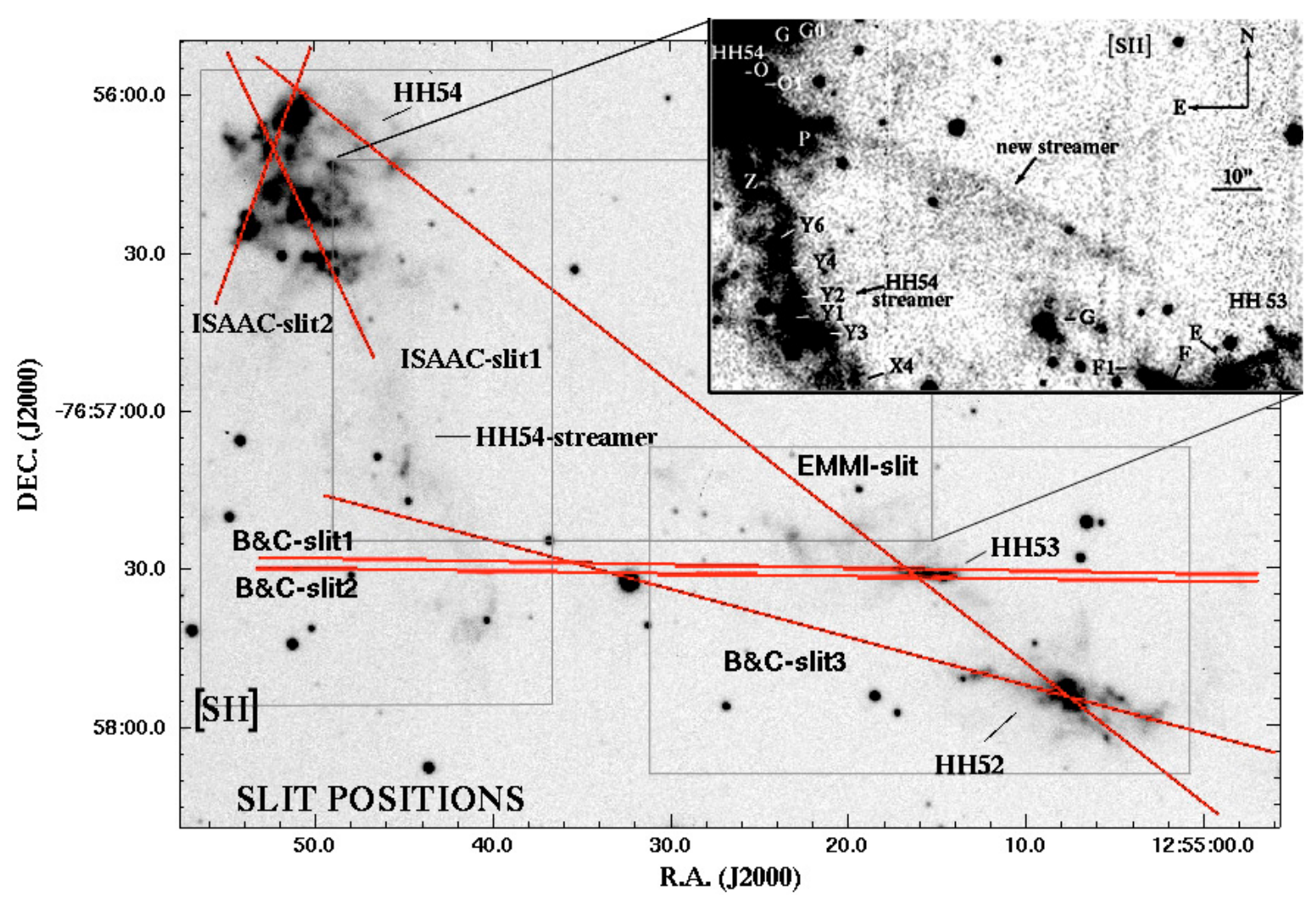

Fig. 1. [S II] EMMI 2006 image of the region indicates the locations of the HH objects, along with EMMI, B\&C, and ISAAC slit positions. In the upper-right inset [S II] deep image (from 1993) we show the newly detected HH 52 streamer. A rectangular box (in the centre of the image) shows its exact location. Additionally, left and right boxes approximatively indicate the fields displayed in Figs. 4-6.

the projected velocities along the line of sight. In order to determine the true 3-dimensional kinematics, i.e. the absolute flow speed and the inclination of the flow with respect to the plane of the sky, proper motion measurements of the knots are also necessary. With this information in hand, a number of important questions can be addressed straightforwardly.

i) The sources of the outflows can be unambiguously identified and knots can be correctly assigned to their driving sources.

ii) Using multi-wavelength data, molecular, and atomic, it is possible to study the different kinematics of the flow by means of tracers that indicate various shock conditions and velocities of the gas.

iii) The internal kinematics of the flows can be studied in detail. Possibly, acceleration, and deceleration can be derived from a multi-epoch analysis of the motions and their origin can be investigated in detail.

iv) Flux variability can be studied and related to the motion variability in the flow.

v) Combining the kinematical results and the gas physical parameters, it is possible to infer the mass-loss rate and the energy budget along the flow. In turn, we can compare these quantities with the physical properties of the exciting source to investigate how it evolves with time.

To address these points, we collected a multi-epoch and multiwavelength (optical and NIR) data archive of several Herbig Haro $(\mathrm{HH})$ objects and protostellar jets. The database includes both imaging and spectroscopy (low and high resolution), mainly covering the optical atomic ( $\mathrm{H} \alpha$, [S II]) and NIR molecular emission $\left(\mathrm{H}_{2}\right)$. For the first time it is possible to study the kinematics of the outflows during an elapse of time up to 20 years at different wavelengths, observing the formation and the evolution of the single structures inside the jets. This paper represents the first work of our project.

The structure is as follows. In Sect. 2 we shortly describe the investigated region. In Sect. 3 our observations are presented. Section 4 reports an overview of our results, including morphology of the flows, their kinematics, flux and velocity variability, physical parameters of the gas, and mass flux rates. An investigation on the possible exciting sources of the flows is presented, as well. In Sect. 5 a discussion is proposed in terms of kinematics vs. flow variability, flow deflection mechanisms, length, and dynamical age of the flows. Our conclusions are summarised in Sect. 6. Finally, a detailed description of our results on the single knots is given in the Appendices.

\section{The investigated region}

HH 52, 53, and 54 are located at the northern edge of the Chamaleon II (Cha II) cloud (Schwartz 1977) at a distance of $178 \pm 18 \mathrm{pc}^{1}$ (Whittet et al. 1997). As shown in Fig. 1, the three $\mathrm{HH}$ objects are roughly placed along a straight path, moving from $\mathrm{SW}$ to $\mathrm{NE}$, with a PA of $\sim 55^{\circ}$. The morphology of the $\mathrm{HH}$ objects is approximatively the same in the $\mathrm{H} \alpha$ and [S II] filters. HH 52 has a bow shock shape pointing towards HH 54. About 20" NE, a chain of bright knots (HH 53 C, A, and B), roughly aligned E-W, is observed. Moreover, farther NE $\left(\sim 1^{\prime}\right)$, a cluster of several bright knots (HH 54) is detected. This group spans some tens of arcseconds and elongates towards SSW, delineating the so-called HH54 streamer

\footnotetext{
${ }^{1}$ Hereafter we refer to this value to compute spatial distances and tangential velocities of the knots.
} 
(see Sandell et al. 1987). In this work, they described the morphology of the $\mathrm{HH}$ objects ${ }^{2}$ in some details, also for the first time reporting a contour map of the molecular hydrogen emission in the NIR. The HHs were spectroscopically observed in the optical by Schwartz \& Dopita (1980) and Graham \& Hartigan (1988), who found only blue-shifted radial velocities ranging from -40 to $-100 \mathrm{~km} \mathrm{~s}^{-1}$. Knee (1992) detected two blue-shifted CO outflows, coinciding with HH 52 and 53, and HH 54 emissions, and only one red-shifted lobe, located approximately between the two. $\mathrm{H}_{2}$ and [Fe II] imaging together with low-resolution spectra of HH 54 were published by Gredel (1994). The morphology in the $\mathrm{H}_{2}$ and [Fe II] filters appears similar to the optical images. More low-resolution spectra in the NIR, with a detailed study of the excitation of the gas in HH 54, have been published by Caratti o Garatti (2006) and Giannini et al. (2006). From these authors $\mathrm{H}_{2}$ medium-resolution spectra of $\mathrm{HH} 54$ and radial velocities are also available. These data cannot explain, however, the kinematics of the object. An optical PM analysis of the HHs with a seven year baseline was attempted by Schwartz et al. (1984) without conclusive results, because of small PM values and relatively large errors.

Finally, the exciting sources of the HHs still remain unclear, even after a proper motion analysis of the infrared knots (Caratti o Garatti et al. 2006), the publication of MIPS maps and YSO catalogues for this region (Young et al. 2005; Porras et al. 2007; Alcalá et al. 2008).

\section{Observations, data reduction and analysis}

Our multi-wavelength database is mainly composed of optical and NIR observations, collected with the ESO facilities during the past twenty years. The relevant information for both imaging and spectroscopy is reported in Tables 1 and 2, respectively. These data were used to derive the kinematics and the physical properties of the studied outflows. The advantage of using a multi-epoch image dataset is quite straightforward: on one hand, we can reduce the errorbars of the PMs and check for velocity variations in the flows; on the other, we can study their flux variability and their evolution with time.

Additionally, this paper makes use of Spitzer Space Telescope public data from the "Cores to Disks" (C2d) Legacy project (Evans et al. 2003), already published and analysed (Young et al. 2005; Porras et al. 2007; Alcalá et al. 2008) in order to infer the exciting sources and map the colder gas component of the outflows.

\subsection{Imaging}

\subsubsection{Optical}

Our optical narrow-band images (see Table 1) are centred on $\mathrm{H} \alpha$ and [S II] emissions. A first set of data was obtained at the ESO/MPG 2.2-m telescope (between 1987 and 1993) with three different detectors: an RCA $30 \mu \mathrm{m}$ CCD chip (1987) (2 images) and an RCA $15 \mu \mathrm{m}$ CCD chip (1989-1990) (4 images), both mounted on the Cassegrain focus, and EFOSC2 (Buzzoni et al. 1984) (1991-1993) (4 images). A second set of images was retrieved at the New Technology Telescope (1995 and 2006) with EMMI (Dekker et al. 1986) (3 images). Because of the relatively small FoV of the CCDs used between 1987 and 1990, HH 52 and HH 53, and HH 54 were observed separately

2 The nomenclature used in this paper mainly refers to Sandell et al. (1987). with the $[\mathrm{S} \mathrm{II}](\lambda \lambda 6716,6731)$ and with the $\mathrm{H} \alpha$ filters, respectively. Starting from 1991, all the three objects were observed in both filters within a single frame. The exposure times range from 1800 to $6000 \mathrm{~s}$, resulting in deep images with a high signal-to-noise ratio $(\mathrm{S} / \mathrm{N})$. The raw data were reduced by using standard procedures in $I R A F^{3}$, subtracting bias, flat-fielding and removing bad pixels. Cosmic rays were removed by using the L.A. Cosmic algorithm (van Dokkum 2001), which was extremely efficient in cleaning cosmic-ray crowded images. The measured seeing on our images ranges between 0.7 and 1..5 (Table 1), with the best values obtained in the most recent ones.

Only images from 2006 have their own photometric standard star, observed with both $\mathrm{H} \alpha$ and [S II] filters. The selected standard is LTT 3218 (Hamuy et al. 1994), for which a flux calibrated spectrum also exists. At the required wavelengths, the flux of the star was obtained by convolving its flux density with the profiles of the ESO filters.

To flux-calibrate the earlier epoch images, 30 bright field stars were selected and photometry in both filters performed on them. For each epoch in each filter, the star fluxes were crosschecked, and those showing variations less than $3 \%$ were chosen as secondary standard stars to calibrate the fields.

\subsubsection{NIR}

Our narrow-band $\mathrm{H}_{2}(2.12 \mu \mathrm{m})$ images were collected during different runs between 1993 and 2005, at the ESO/MPG 2.2-m telescope with IRAC2b (Lidman et al. 1998a) (3 images), at the ESO-NTT with SofI (Moorwood et al. 1998a) (2 images), and at ESO-VLT with ISAAC (Moorwood et al. 1998b) (2 images) (see Table 1). Some data on HH 54 have been presented in previous papers (Giannini et al. 2006; Caratti o Garatti et al. 2006), where PMs were derived comparing the SofI image of June 1999 with the ISAAC one of January 2005. Here, we enlarge the baseline of our observations including IRAC2b data from 1993 and 1995, and an ISAAC image from 2002 (covering only HH 54) that, on one hand, allow us to also derive $\mathrm{H}_{2}$ PMs for $\mathrm{HH} 52$ and $\mathrm{HH} 53$, and, on the other, add more data points to HH 54, reducing the uncertainties on PMs and PAs of the knots. The first set of IRAC2b data (1993) covers HH 52 and HH 53, and HH 54, separately, exhibiting a poor seeing of $1^{\prime \prime} .7$. The second (1995) consists of a mosaic of the region $\left(\sim 7^{\prime} \times 7^{\prime}\right)$ and has a seeing of 1 .' 1 . In addition, more $\mathrm{H}_{2}$ SofI data from the ESO science archive facility ${ }^{4}$ (taken less then 3 months apart from those of June 1999, see Table 1) were retrieved and coadded to create a deeper SofI $\mathrm{H}_{2}$ map of the region covering $\sim 11^{\prime} \times 11^{\prime}$.

Finally, additional $\mathrm{H}_{2}$ and $K_{\mathrm{S}}$ images around IRAS 125007658 , one of the possible driving sources of HH 54 (see also Sects. 3.1.3 and 4.9), were taken in Jan. 2007 to detect a possible $\mathrm{H}_{2}$ jet from that source.

As for the optical, all the raw data were reduced by using IRAF packages applying standard procedures for sky subtraction, dome flat-fielding, bad pixel and cosmic ray removal and image mosaicing. Flux calibration by means of a photometric standard star was only possible for the June 1999 SofI image. As for the optical, the remaining $\mathrm{H}_{2}$ images were calibrated by selecting a few field stars that showed flux variations smaller than $10 \%$.

\footnotetext{
3 IRAF (Image Reduction and Analysis Facility) is distributed by the National Optical Astronomy Observatories, which are operated by AURA, Inc., cooperative agreement with the National Science Foundation.

${ }^{4}$ http://archive.eso.org/
} 
Table 1. Journal of observations - Imaging.

\begin{tabular}{ccccccc}
\hline \hline $\begin{array}{c}\text { Date of obs. } \\
(\mathrm{d}, \mathrm{m}, \mathrm{y})\end{array}$ & $\begin{array}{c}\text { Telescope/ } \\
\text { instrument }\end{array}$ & $\begin{array}{c}\text { Filter } \\
\text { band }\end{array}$ & $\begin{array}{c}\text { Resolution } \\
\left({ }^{\prime \prime} / \mathrm{pixel}\right)\end{array}$ & $\begin{array}{c}\text { Seeing } \\
\left({ }^{\prime \prime}\right)\end{array}$ & $\begin{array}{c}\text { Exp. time } \\
(\mathrm{s})\end{array}$ & $\begin{array}{c}\text { Notes } \\
\text { objects observed }\end{array}$ \\
\hline 14.05 .2006 & ESO-NTT/EMMI & $\mathrm{H} \alpha$ & 0.166 & 0.8 & 3600 & HH52, HH53, HH54 \\
12.05 .1995 & ESO-NTT/EMMI & $\mathrm{H} \alpha$ & 0.266 & 1.1 & 3600 & HH52, HH53, HH54 \\
31.03 .1993 & ESO/MPG2.2-m/EFOSC2 & $\mathrm{H} \alpha$ & 0.35 & 0.9 & 4500 & HH52, HH53, HH54 \\
08.05 .1992 & ESO/MPG2.2-m/EFOSC2 & $\mathrm{H} \alpha$ & 0.35 & 1.4 & 5400 & HH52, HH53, HH54 \\
15.01 .1990 & ESO/MPG2.2-m/CCD & $\mathrm{H} \alpha$ & 0.35 & 1.0 & 6000 & HH54 \\
03.02 .1989 & ESO/MPG2.2-m/CCD & $\mathrm{H} \alpha$ & 0.35 & 1.0 & 4200 & HH54 \\
04.03 .1987 & ESO/MPG2.2-m/CCD & $\mathrm{H} \alpha$ & 0.35 & 1.2 & 3600 & HH54 \\
14.05 .2006 & ESO-NTT/EMMI & {$[\mathrm{S} \mathrm{II]}$} & 0.166 & 0.7 & 3600 & HH52, HH53, HH54 \\
31.03 .1993 & ESO/MPG2.2-m/EFOSC2 & {$[\mathrm{S} \mathrm{II]}$} & 0.35 & 1.0 & 4200 & HH52, HH53, HH54 \\
09.02 .1991 & ESO/MPG2.2-m/EFOSC2 & {$[\mathrm{S} \mathrm{II]}$} & 0.35 & 1.1 & 1800 & HH52, HH53, HH54 \\
14.01 .1990 & ESO/MPG2.2-m/CCD & {$[\mathrm{S} \mathrm{II]}$} & 0.35 & 0.8 & 4800 & HH52, HH53 \\
01.02 .1989 & ESO/MPG2.2-m/CCD & {$[\mathrm{S} \mathrm{II]}$} & 0.35 & 1.2 & 3600 & HH52, HH53 \\
28.02 .1987 & ESO/MPG2.2-m/CCD & {$[\mathrm{S} \mathrm{II]}$} & 0.35 & 1.5 & 3600 & HH52, HH53 \\
01.01 .2005 & ESO-VLT/ISAAC & $\mathrm{H}_{2}$ & 0.148 & 0.5 & 40 & HH54 \\
02.07 .2002 & ESO-VLT/ISAAC & $\mathrm{H}_{2}$ & 0.148 & 0.5 & 60 & HH54 \\
06.06 .1999 & ESO-NTT/SofI & $\mathrm{H}_{2}$ & 0.29 & 1.0 & 300 & HH52, HH53, HH54 \\
31.03 .1999 & ESO-NTT/SofI & $\mathrm{H}_{2}$ & 0.29 & 0.9 & 600 & HH52, HH53, HH54 \\
15.03 .1995 & ESO/MPG2.2-m/IRAC2b & $\mathrm{H}_{2}$ & 0.51 & 1.1 & 500 & HH52, HH53, HH54 \\
10.04 .1993 & ESO/MPG2.2-m/IRAC2b & $\mathrm{H}_{2}$ & 0.51 & 1.7 & 200 & HH54 \\
03.04 .1993 & ESO/MPG2.2-m/IRAC2b & $\mathrm{H}_{2}$ & 0.51 & 1.7 & 200 & HH52, HH53 \\
01.09 .2007 & ESO-NTT/SofI & $\mathrm{H}_{2}$ & 0.29 & 1.0 & 1080 & IRAS 12500-7658 \\
01.09 .2007 & ESO-NTT/SofI & $K_{\mathrm{s}}$ & 0.29 & 1.0 & 270 & IRAS 12500-7658 \\
\hline
\end{tabular}

Table 2. Journal of observations - Spectroscopy.

\begin{tabular}{ccccccc}
\hline \hline $\begin{array}{c}\text { Telescope/ } \\
\text { Instrument }\end{array}$ & $\begin{array}{c}\text { Date of obs. } \\
(\mathrm{d}, \mathrm{m}, \mathrm{y})\end{array}$ & $\begin{array}{c}\text { Wavelength } \\
(\AA)\end{array}$ & $\begin{array}{c}t_{\text {int }} \\
(\mathrm{s})\end{array}$ & $\begin{array}{c}\text { PA } \\
\left({ }^{\circ}\right)\end{array}$ & $\mathcal{R}$ & Encompassed knots \\
\hline ESO-NTT/ & 14.05 .2006 & $6200-7000$ & 2700 & 52 & 3000 & HH52: A3-A4, A1, D4; HH53: C-C1, E1; \\
EMMI & & & & & & HH54: G-G0, G1-G3, C2, C3 \\
ESO-VLT/ISAAC & 01.01 .2005 & 16400 & 3600 & 29.5 & 10000 & HH54: Z, A3, A1, B, J-J1, H3, H2 \\
ESO-VLT/ISAAC & 01.01 .2005 & 16400 & 3600 & 160.5 & 10000 & HH54: C3, C1, H3, J-J1, E, K \\
ESO-2.2-m/ & 20.02 .1987 & $6200-6800$ & 3600 & 89.5 & 2000 & HH53: B, A, C, F2; HH54: X4 C, X1 D \\
Boller \& Chivens (B\&C) & 21.02 .1987 & $6200-6800$ & 3600 & 89.5 & 2000 & HH53: B, A, C; HH54: X3, X1 A \\
& 25.02 .1987 & $6200-6800$ & 3600 & 75 & 2000 & HH52: B, A, D3-D2; HH54: X4 B-A \\
\hline
\end{tabular}

\subsubsection{Spitzer data}

Reduced Spitzer data have been obtained by the public catalogue c2d (third delivery). They consist of large mosaics of the Cha II cloud observed with the Infrared Array Camera (IRAC) in four channels (at 3.6, 4.5, 5.8, and $8.0 \mu \mathrm{m})\left(\sim 1.04 \mathrm{deg}^{2}\right)$ and with MIPS (at 24, $70 \mu \mathrm{m})\left(\sim 1.5 \mathrm{deg}^{2}\right)$. In Fig. 2 we report part of the Spitzer-MIPS map (at $24 \mu \mathrm{m}$ ), indicating the location of the studied $\mathrm{HH}$ objects and the possible driving sources.

\subsubsection{Knot identification and flux analysis}

To identify the knots along the flows, we used the best image in each filter (i.e. with highest $\mathrm{S} / \mathrm{N}$ and best resolution). This resulted to be the last epoch image (i.e. EMMI 2006 image in $\mathrm{H} \alpha$ and [S II], SofI 1999 image for HH 52 and HH 53 in $\mathrm{H}_{2}$, and ISAAC 2005 image for $\mathrm{HH} 54$ in $\mathrm{H}_{2}$ ). These were also used as reference images for the PM analysis (see Sect. 3.1.5). Each structure was then identified and labelled (in each filter) within a $5 \sigma$ contour around the local maximum. These contours were also used to measure the total flux of the knots in each epoch and filter, and to study the flux variability as well.

\subsubsection{PM analysis}

Particular attention was paid to align the images. In fact, an accurate determination of the proper motions requires that the multi-epoch images have to be scaled and then registered to a subpixel accuracy in each filter. Our observed region contains several field stars; therefore, the image registration was easy to implement, and the distortions introduced by the different optical systems were corrected by means of geomap (with a polynomial fit of 3rd order) and geotran routines in IRAF. Because of the large baseline of the observations, few stars showed a significant proper motion and were excluded from the sample. Each image in each filter was aligned to a common (scaled) reference frame (see Sect. 3.1.4). The resulting error is given by the residuals of the fit. Statistical errors in the optical were typically about $\pm 0.1-0.2$ pixels (i.e. $\sim 0$ ' $^{\prime} 03-00^{\prime} 07$ ), depending on the seeing and on the resolution of the image. In the NIR, due to the reduced FoV, ISAAC, and IRAC2b (1993) images have an exiguous number of stars $(\leq 20)$ that diminishes the accuracy in the alignment, not allowing the entire area to be sampled homogeneously. As a consequence, here the errors were up to $\pm 0.3-0.4$ pixels.

Knot shifts were determined between image pairs (i.e. last epoch and previous epoch images) using a cross-correlation 


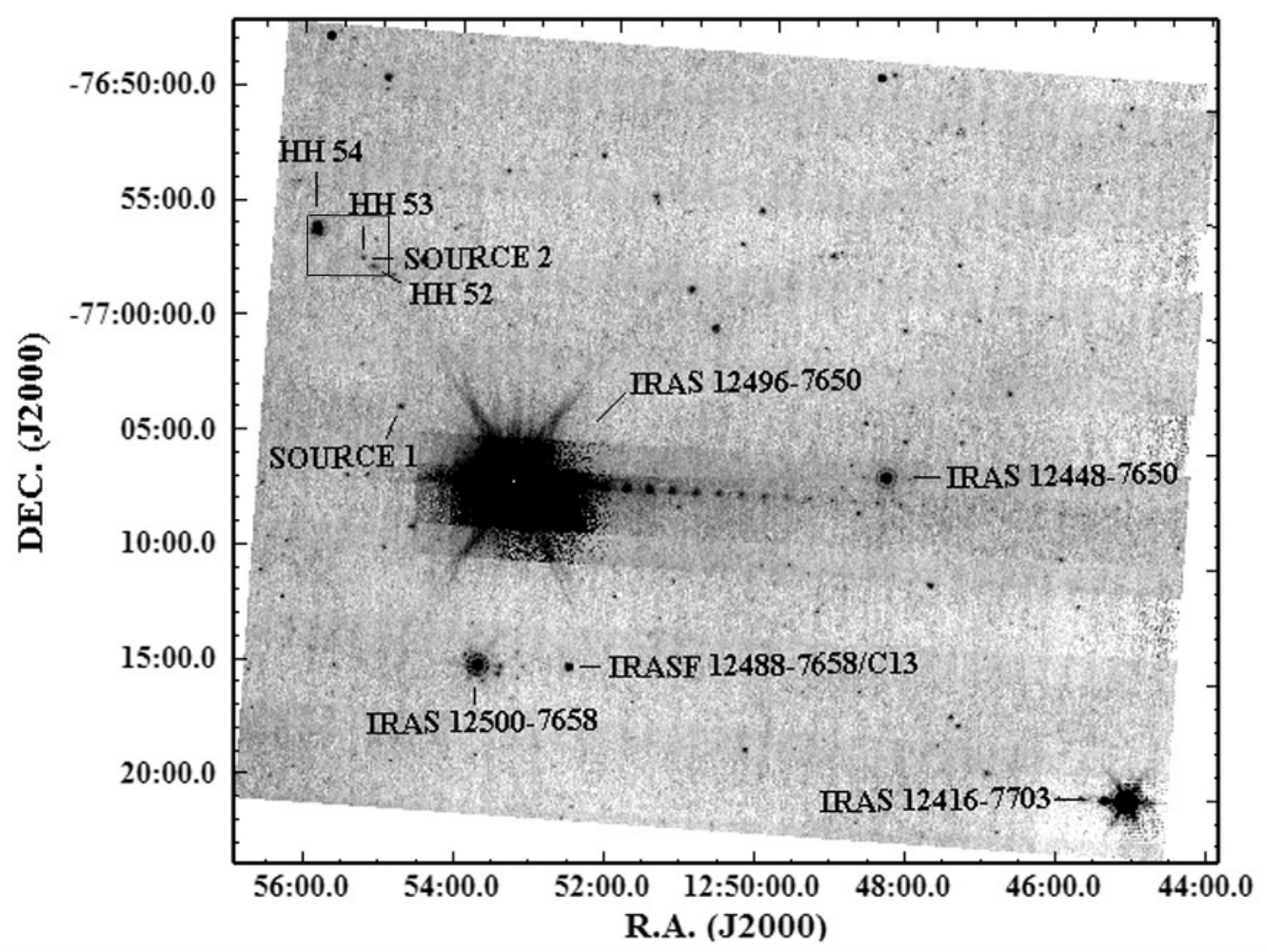

Fig. 2. Spitzer-MIPS $24 \mu \mathrm{m}$ map of the investigated Cha II region. The candidate exciting sources and the HH objects are reported. A rectangular box indicates the region displayed in Fig. 1.

method. A rectangle was defined round each knot, enclosing its $5 \sigma$ contour. The 1 st-epoch image was then shifted with subpixel accuracy (0.1 pix) with respect to the last epoch image and then multiplied by it. For each shift $(x, y)$, the total integrated flux $(f)$ in the rectangle around each knot was measured in the product image. As the final shift for each knot, we used the position of the maximum of $f(x, y)$, determined via a Gaussian fit. For each structure, an initial guess of the shift vector was estimated visually by blinking the image pairs, defining a maximum shift for each rectangle. This served as input into our routine for the cross-correlation. For several features, the analysis routine converged rapidly to a solution, independent of the initial guess. A more accurate estimate (within a few pixels) of the size and direction of the proper motion was needed to avoid the code to correlate spurious pairs in case of diffuse knots located close to brighter knots or stars. To prove the code accuracy, we tested it on both nebulous and stellar objects by shifting the images and recovering the offsets with the code.

To quantify the systematic errors for the shift measurements, we varied the size and shape of the rectangle that defines the object. The resulting range of values gives a systematic error, which depends on the $\mathrm{S} / \mathrm{N}$, shape, and time variability of the knot. This error is comparable to or even larger than the alignment error. Thus, each single shift error was derived combining both the alignment error of the two epoch images and the uncertainty in the centring routine. Usually, for each pair, the final error was around $10 \%$ of the PM value. However, for slow knots with a low $\mathrm{S} / \mathrm{N}$, the error was up to $100 \%$.

The final PM value and error for each knot in each filter were obtained from a weighted least square fit of the shifts, fitting the motion in X (RA) and Y (Dec) simultaneously (see Fig. 3), using the equations of both linear and accelerated motions: $[x(t), y(t)]=p_{x, y}+v_{x, y} t+\left(\frac{1}{2} a_{x, y} t^{2}\right)$. Both linear and quadratic fits
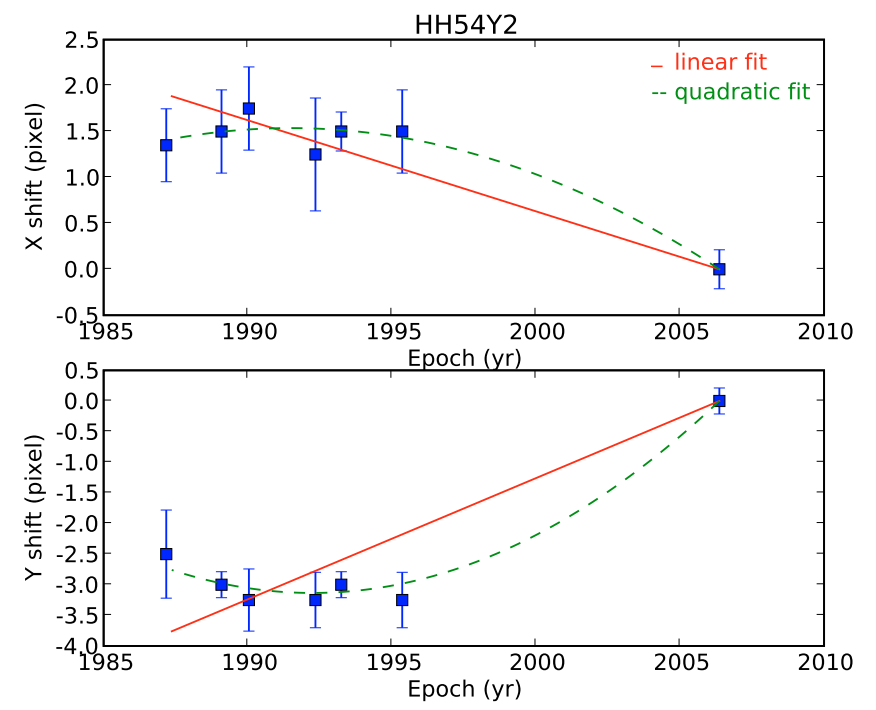

Fig. 3. Example of linear and quadratic fits performed on the dataset to derive proper and accelerated motions. Here the fits on HH 54 Y2 $\mathrm{H} \alpha$ data are reported.

(for knots with a number of epochs $\geq 3$ ) were performed to detect any possible acceleration or deceleration of the knot, as well. The resulting errors on proper and accelerated motions were derived from the fits. An example of our fits is shown in Fig. 3, where an accelerated motion is detected. The upper and lower panels report the $\mathrm{X}$ and $\mathrm{Y}$ shifts with errors for the $\mathrm{H} \alpha$ filter of HH 54 Y2, respectively. Straight and dashed lines indicate linear and quadratic fits. In this particular case both $\mathrm{X}$ and $\mathrm{Y}$ shifts are better modelled by a quadratic fit. 


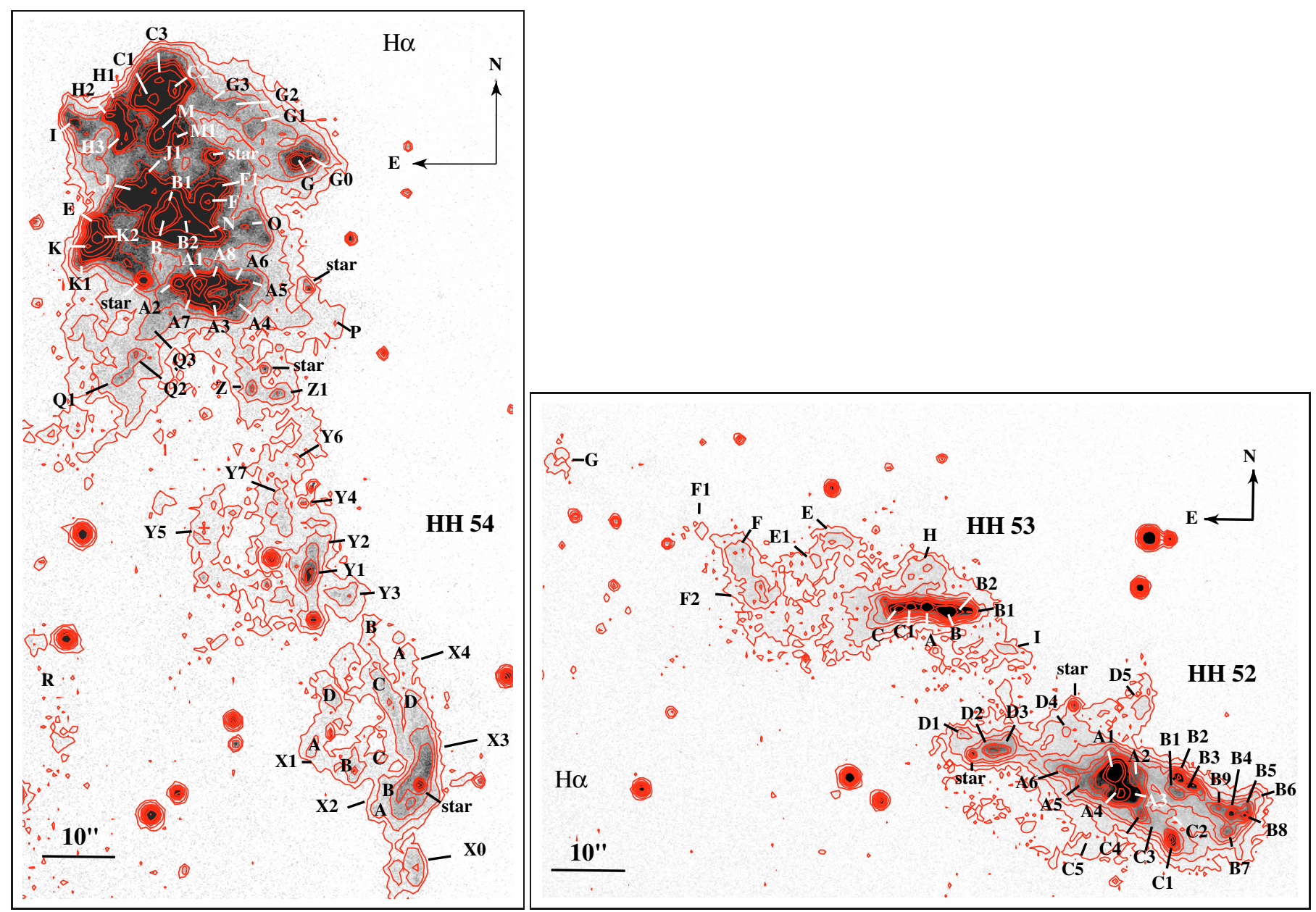

Fig. 4. H $\alpha$ images (EMMI 2006) of HH 54 (left panel), HH 52 and HH 53 (right panel). The labels indicate the position of the knots, including the newly detected ones. The contour levels are $3,10,20,30,40,50,60,70,80,90,100 \times$ the standard deviation to the mean background $(\sigma \sim 4 \times$ $\left.10^{-17} \mathrm{erg} \mathrm{s}^{-1} \mathrm{~cm}^{-2} \operatorname{arcsec}^{-2}\right)$.

\subsection{Spectroscopy}

\subsubsection{Optical}

Four optical medium-resolution spectra ( $\mathcal{R} \sim 2000-3000)$ were collected with a Boller \& Chivens (B\&C) spectrograph at the ESO/MPG 2.2-m telescope (1987) and with EMMI at the NTT (2006). They range from 6200 to $7000 \AA$ and cover the spectral region containing bright lines from [O I] (6300 $\AA$ and $6363 \AA$ ), [N II] (6548 $\AA$ and $6583 \AA), H \alpha(6563 \AA)$, and [S II] (6716 $\AA$ and $6731 \AA$ ), widely used for the diagnostics of the shocked gas (see e.g. Bacciotti \& Eislöffel 1999, hereafter BE99). The observed targets and the slit PA are listed in Table 2, together with the date of the observations and the total integration time. B\&C slit 1 and 2 are positioned on the three $\mathrm{HH} 53$ bright knots $(\mathrm{A}-\mathrm{C})$ (see also Fig. 1), and intersect the HH 54 streamer. They have the same PA $\left(89^{\circ} .5\right)$ and almost the same positions, with slit 1 shifted slightly northward and also encompassing knots F2 in HH 53 and X4 C and X1 D in HH 54, while slit 2 covers knots X3 and X1 A of HH54 streamer (see Fig. 4 and Appendix A for a detailed description of the flow morphology). $\mathrm{B} \& \mathrm{C}$ slit 3 (PA = $75^{\circ}$ ) is placed on HH 52 A, B and D2-D3, and also includes knots $\mathrm{X} 4 \mathrm{~A}$ and $\mathrm{B}$ in HH 54 streamer. Finally, EMMI slit $\left(\mathrm{PA}=52^{\circ}\right)$ covers HH 52 B, A, D3-D2, HH 53 C and C1, HH 54 G, and C. The total integration time for B\&C and EMMI spectra are $3600 \mathrm{~s}$ and $2700 \mathrm{~s}$, respectively. We used the same procedure as for the optical imaging to reduce the data. A first rough wavelength calibration was retrieved from helium and argon lamps. A second, more accurate calibration was done using several atmospheric $\mathrm{OH}$ lines (Osterbrock et al. 1996) present in each frame with a final accuracy of $4 \mathrm{~km} \mathrm{~s}^{-1}$ in the EMMI spectrum. LTT 7379 was the standard star observed to flux calibrate the EMMI spectrum. The B\&C data were calibrated using our narrow-band images.

\subsubsection{NIR}

Two medium-resolution spectra around the $1.64 \mu \mathrm{m}$ [Fe II] line $\left(\lambda_{\mathrm{vac}}=1.64399 \mu \mathrm{m}\right.$; Johansson 1978) were obtained with the ISAAC IR camera, with a 0.3 slit, corresponding to a nominal resolving power of $\sim 10000$. Targets and PAs of the slits are reported in Table 2, while the slit positions are shown in Fig. 1 (ISAAC slit 1 and 2). The two slits encompass only HH 54. The first slit $\left(\mathrm{PA}=29^{\circ} .5\right)$ encompasses knots A3, A1, B3, B1, partially $\mathrm{J}$ and $\mathrm{J} 1, \mathrm{H} 3$, and $\mathrm{H} 2$. The second slit $\left(\mathrm{PA}=160^{\circ} .5\right)$ is centred on knots $\mathrm{K} 1, \mathrm{~K}$, and $\mathrm{E}$, but partially encompasses knots $\mathrm{J}$, $\mathrm{J} 1, \mathrm{H} 3, \mathrm{C} 1$, and $\mathrm{C} 3$.

To perform our spectroscopic measurements, we adopted the usual ABB "A" configuration, for a total integration time of $3600 \mathrm{~s}$. Each observation was flat-fielded, sky-subtracted, and corrected for the curvature derived by longslit spectroscopy, while atmospheric features were removed by dividing the spectra by a telluric standard star (O8 spectral type), normalised 
to the blackbody function at the stellar temperature and corrected for hydrogen recombination absorption features intrinsic to the star. The wavelength calibration was performed using atmospheric $\mathrm{OH}$ lines (Rousselot et al. 2000) with an accuracy of $3 \mathrm{~km} \mathrm{~s}^{-1}$. The instrumental profile in the dispersion direction, measured from Gaussian fits to the sky $\mathrm{OH}$ emission lines, was $38.0 \mathrm{~km} \mathrm{~s}^{-1}$.

\section{Results}

\subsection{Morphology}

An overview of the HH 52, 53, and 54 regions is given in Fig. 1. Additionally, in Figs. 4, A.1, and A. $2^{5}$ we present $\mathrm{H} \alpha$, [S II], and $\mathrm{H}_{2}$ images of $\mathrm{HH} 52, \mathrm{HH} 53$, and $\mathrm{HH} 54$ with labels indicating the position of the knots, including the newly detected ones. Their coordinates are reported in Cols. 2 and 3 of Tables B. $1-$ B. $4^{6}$. Note that some emissions are not visible in all the three filters. The atomic and molecular emissions only partially overlap, sometimes showing a different spatial distribution of the gas in each filter that indicates various excitational conditions along the flows.

A first glance to Fig. 1 reveals at least two flows converging towards HH 54 brightest region, which shows a chaotic structure (see also Fig. 4). A first, well-known outflow, delineated by the HH 54 streamer, is oriented NNE with a position angle of $\sim 22^{\circ}$ (see also Fig. 4). A second outflow follows an NE direction with a position angle of $50^{\circ}-55^{\circ}$, grouping $\mathrm{HH} 52,53$, and 54 . In our images we detect a new streamer (hereafter $\mathrm{HH} 52$ streamer, see upper-right panel in Fig. 1), which connects the HH 52 bow shock to the main body of HH54 and it is superimposed on $\mathrm{HH}$ 53. The structure is barely visible in $\mathrm{H}_{2}$, but well delineated in our deepest [S II] and $\mathrm{H} \alpha$ images. It is detected in the Spitzer images as well. It appears as continuous slightly curved emission between HH 54 and 53 knots, while it is fragmented in single knots between HH 53 and 52. Both the narrow-band images and the EMMI spectrum seem to indicate that the HH 52 streamer only presents line emission.

We do not detect any other additional emission in the large $\mathrm{H}_{2}$ SofI map of the region down to the $3 \sigma$ limit of $\sim 3 \times 10^{-16} \mathrm{erg} \mathrm{s}^{-1} \mathrm{~cm}^{-2} \operatorname{arcsec}^{-2}$. Neither do we detect any jet or molecular emission in the $2.12 \mu \mathrm{m}$ image around IRAS 12500-7658 (a possible driving source of HH54 - see also Fig. 2 and Sect. 4.9) down to a $3 \sigma$ limit of $\sim 1.5 \times$ $10^{-15} \mathrm{erg} \mathrm{s}^{-1} \mathrm{~cm}^{-2} \operatorname{arcsec}^{-2}$. Moreover, we do not observe any further emission in the Spitzer large field images, with the exception of a faint nebulosity in IRAC band $2(4.5 \mu \mathrm{m})$ located south $\sim 2$ !. 8 SSE of HH 52 (at about $\alpha=12^{\mathrm{h}} 54^{\mathrm{m}} 50^{\mathrm{s}}$ and $\delta=-77^{\circ} 00^{\prime} 37^{\prime \prime} .2$ ) and extending approximatively $\sim 1^{\prime}$ towards IRAS $12496-7650$, located $\sim 8^{\prime}$ farther SSE. It is not clear, however, whether this emission is correlated with the outflows.

Finally, in the Appendix A, for each object, a detailed description of the morphology is reported.

\subsection{Proper motions}

In this section we report the general results of our proper motion analysis. A detailed description for each $\mathrm{HH}$ object is given separately in Appendix B. During the time interval of our observations, some objects showed high degrees of variability, considerably changing in velocity, direction, shape, and flux.

\footnotetext{
5 Figures A.1 and A.2 are presented in Appendix A.

6 Tables B.1-B.4 are presented in Appendix B.
}

Sometimes it was possible to observe the formation or the vanishing of structures along the flows. Therefore, we leave to Sects. 4.3, 4.4, and to Appendix C the detailed report of these phenomena.

In Figs. 5, 6, and B. $1^{7}$, we show the flow charts of the two regions for the three filters ( $\mathrm{H} \alpha$, [S II], and $\mathrm{H}_{2}$, respectively). The PMs and their error bars are indicated by arrows and ellipses, respectively. The results of our PM measurements (from the linear fits) are given as online material in Appendix B. Tables B.1 (HH 52 and 53) and B.2 (HH 54) report the values of the optical lines, and Tables B.3 (HH 52 and 53) and B.4 (HH 54) those of the $\mathrm{H}_{2}$ line. Each table lists the knot ID, the coordinates, the measured proper motion from the linear fit, the derived tangential velocity $\left(v_{\tan }\right)$ at a distance of $178 \mathrm{pc}$, and the filter used. Where PMs could not be measured only the knot ID, the coordinates, and the filter (in which the knot was detected) are reported. Those data which are better modelled by a quadratic fit are labelled with an "a", and the results of the quadratic fits are listed in Table C. $1^{8}$ (see Sect. 4.3).

With few exceptions, the derived PMs have similar values in the optical filters, with values ranging between 0.007 and $0.097^{\prime \prime} \mathrm{yr}^{-1}$, corresponding to a $v_{\tan }$ between 6 and $82 \mathrm{~km} \mathrm{~s}^{-1}$. On average, the $\mathrm{H}_{2}$ PMs have lower values, ranging between 0.007 and $0.041^{\prime \prime} \mathrm{yr}^{-1}$ (or 6 and $35 \mathrm{~km} \mathrm{~s}^{-1}$ ), and the uncertainties are usually greater, especially for the HH 52 and 53 regions, due to the smaller number of epochs, poor seeing, lower resolution of the used instruments, and less accuracy in the alignment (see also Sect. 3.1.2).

A quick inspection of HH 52 and 53 (right panel of Figs. 5 and 6) reveals the presence of two different outflows. A first flow (hereafter HH 52 flow) is composed of the majority of the knots (those of HH 52 and several of $\mathrm{HH} 53$, i.e. from $\mathrm{C} 1$ to $\mathrm{G}$ ). It moves NE with a position angle of $\sim 55^{\circ}$ (towards HH 54), roughly following the path delineated by the HH 52 streamer and partially superposing on a second flow (hereafter HH 53 flow). This one, made of HH 53 A, B, B1, and C, has approximatively an E-W direction and does not seem to be related to the HH 52 flow. The features of the HH 52 flow are moving at a $v_{\tan }$ between $\sim 30$ and $60 \mathrm{~km} \mathrm{~s}^{-1}$, where the different velocities do not seem to be associated with the position of the objects along the flow, but rather they could originate from local interactions inside the fluid. On the contrary, HH 53 A, B and C, in the HH 53 flow, exhibit smaller PMs $\left(0.01-0.02^{\prime \prime} \mathrm{yr}^{-1}\right.$ or $9-18 \mathrm{~km} \mathrm{~s}^{-1}$ ) with PAs around $270^{\circ}$, indicating that they are probably part of a different outflow.

The left panels of Figs. 5 and 6 reveal the complex kinematics of HH 54. The trajectories of the knots indicate that at least two distinct outflows are present in this region and they appear to be superimposed on the main body of HH 54. A first flow is generated by the HH 54 streamer (hereafter HH 54 flow) and is moving NNE $\left(\mathrm{PA} \sim 20^{\circ}\right)$. It includes knot groups $\mathrm{X}, \mathrm{Y}$ and $\mathrm{Z}$ (HH 54 streamer) and likely groups $\mathrm{E}, \mathrm{K}$ and $\mathrm{Q}$ (in the main body), with the $v_{\tan }$ that decreases from $\sim 60$ to $\sim 10 \mathrm{~km} \mathrm{~s}^{-1}$ moving from the stream to the main body. A second outflow with an $\mathrm{NE}$ direction $\left(\mathrm{PA} \sim 55^{\circ}\right)$ is the extension of the HH 52 flow and contains groups $\mathrm{G}, \mathrm{C}$, and, possibly, A, which appears deflected $\left(\mathrm{PA} \sim 80^{\circ}, v_{\tan } \sim 30 \mathrm{~km} \mathrm{~s}^{-1}\right.$ ). Indeed, for several knots the affiliation with one of the two flows cannot be certain, and the presence of other flows, even if unlikely, cannot be ruled out (see also Appendix $\mathrm{C}$ for a detailed discussion). This is particularly evident in the central part of the $\mathrm{HH}$ object, where

\footnotetext{
7 Figure B.1 is presented in Appendix B .

8 Table C. 1 is presented in Appendix C.
} 


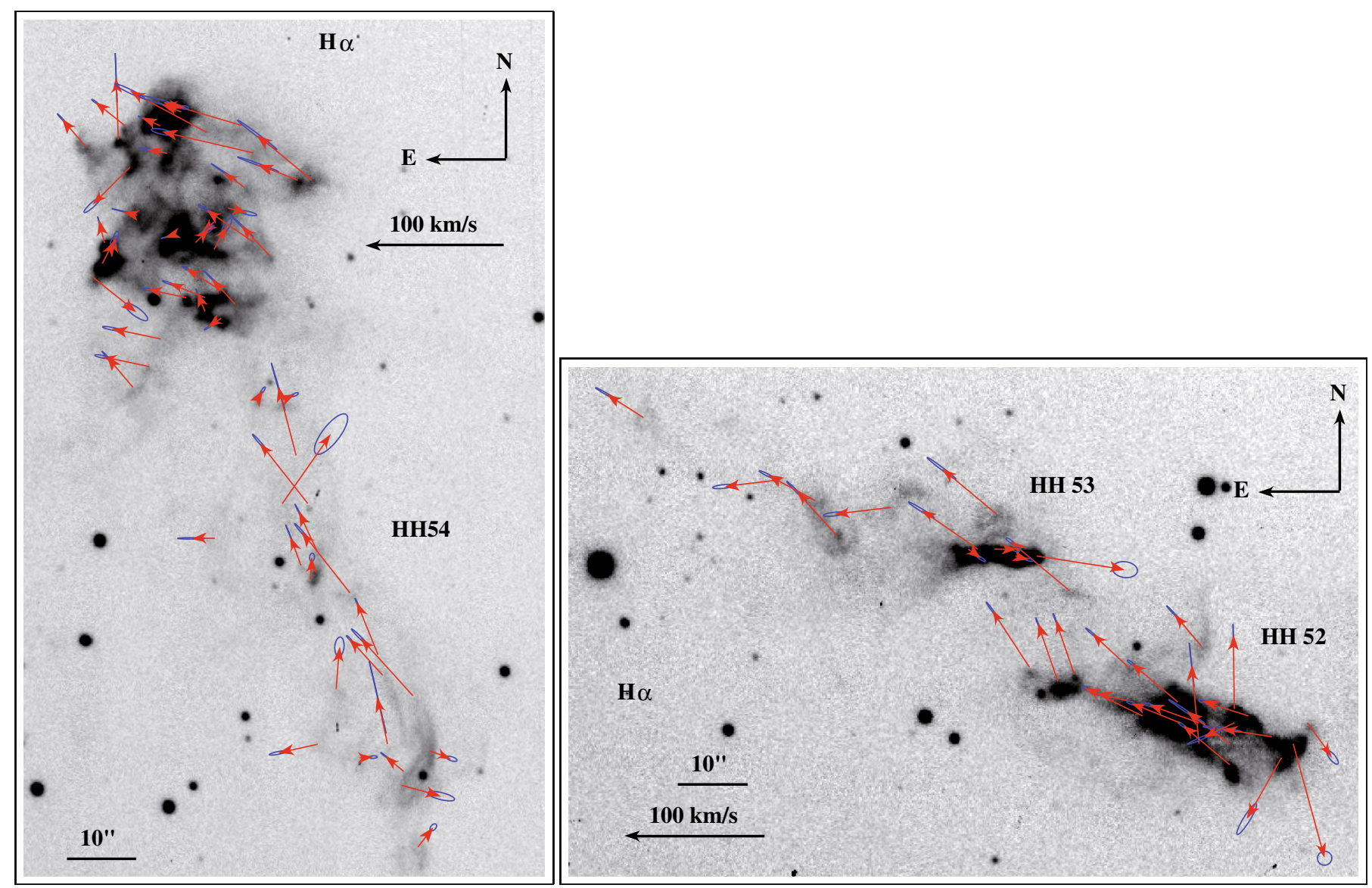

Fig. 5. Flow charts of HH 54 (left panel), HH 52 and 53 (right panel) in the $\mathrm{H} \alpha$ filter. Proper motions and their error bars are indicated by arrows and ellipses, respectively.

the overlap of the flows and the low PM values make it difficult to distinguish between real and apparent motions.

\subsection{Accelerated motions}

As anticipated in the previous section, Table C.1 reports those datasets that are better modelled by a quadratic fit. In particular, the table lists, the knot ID; the goodness of the chi-square for the quadratic fit $\left(P_{a}\left(\chi^{2} / v\right)\right.$, i.e. the 1 -tail probability value associated with the provided chi-square value and degrees of freedom $v$ ); the proper and accelerated motions derived from the fit; the PAs of the PM and of the acceleration vector; the filter and the notes. The data have been selected by following criteria: a) $P\left(\chi^{2} / v\right)$ of the quadratic fit is greater than that of the linear fit; $b) P_{a}\left(\chi^{2} / v\right) \geq$ $0.05 ; c)$ the measured accelerated motion is $\geq 2 \sigma$. For datasets with only three measurements, conditions $a$ and $b$ are always verified with $P_{a}\left(\chi^{2} / v\right)=1$. In this case we report the result of the quadratic fit only to support or reject the evidence of an accelerated motion measured in other filters. It is worth to note that same or opposite directions of proper and accelerated motion (i.e. PA values of $\boldsymbol{v}$ and $\boldsymbol{a}$, respectively) indicate acceleration or deceleration of the knot. As a result of this selection, Table C.1 lists 33 (out of 108) knots that seem to show some velocity variability in at least one filter. However, not all of these results seem to be genuine. For example, a few knots (i.e. HH 52 A5, $53 \mathrm{G}$, $54 \mathrm{G} 2,54 \mathrm{H} 3$, see Table C.1) have inconsistent $\operatorname{PA}(\boldsymbol{a})$ values in different filters, whereas we would expect similar dynamics for the same knot even with different filters (i.e. it is unlikely that the same knot is accelerated in a filter and decelerated in the other).
Moreover the PA(v) of some other knots appears to be inconsistent with respect to the PA measured from the linear analysis, and with respect to the PA that is expected from the geometry of the flow. We labelled all these knots as "no" in the notes of Table C.1. Indeed for these knots the quadratic fits do not represent a reliable physical solution. After this further selection, only 17 knots are left. They indeed appear to be genuine accelerated motions. Among them, 12 objects accelerate and 5 decelerate. For most of them, both modules and PAs of proper and accelerated motions are identical (inside the errors) for the two optical filters.

\subsection{Flux, direction, and velocity variability}

In this section we report some examples of the observed time variability in flux, velocity, and direction. In Appendix C we provide details on those most significant.

On average, the analysed knot fluxes are variable with time. About $60 \%$ of the analysed knots (62 out of 108) showed a variability above the $3 \sigma$ error bars. However, such variations often $(\sim 60 \%)$ do not have a particular trend, but rather appear as temporary fluctuations around a mean value. For these objects, the PMs, inside the error bar, are usually constant with time. On the other hand, in 20 years about $20 \%$ of the knots (i.e. 19 out of 108) showed velocity variability, together with flux variations.

The structures that show the highest variability in morphology, flux, and velocity can be mostly identified with (1) working surfaces or (2) interacting knots, where the faster fluid particles ejected at later times eventually catch up with the slower flow 


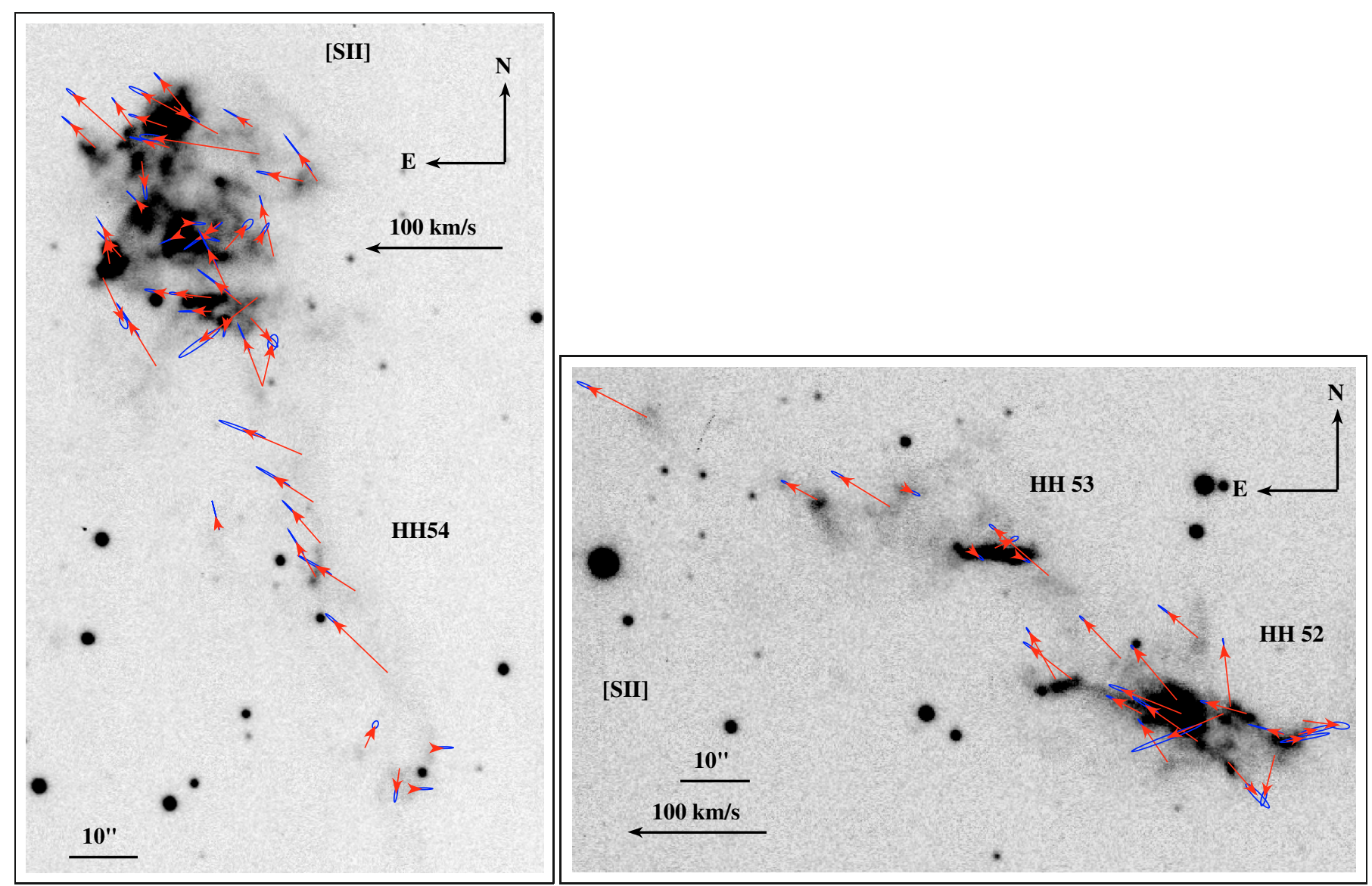

Fig. 6. Flow charts of HH 54 (left panel), HH 52 and 53 (right panel) in the [S II] filter. Proper motions and their error bars are indicated by arrows and ellipses, respectively.

ejected earlier from the source. Usually, the fast gas from behind is decelerated, while the slow gas in front is accelerated. This phenomenon is mostly accompanied by flux variability. In particular, we identify four regions that clearly illustrate such a phenomenon: HH $52 \mathrm{~B}$ and C, HH $54 \mathrm{G}$, and HH $54 \mathrm{H}$.

For the analysed knots, the largest flux variations have been usually detected in the $\mathrm{H} \alpha$ and $\mathrm{H}_{2}$ emissions, while the [S II] usually presents a lower degree of flux variability. Also the timing and the duration of such a variability seem to be different in the three filters. In particular, the optical component seems to excite first and then raises the molecular emission, which usually seems to exhibit a longer cooling time ( $\geq 10$ years). This is, however, a very qualitative analysis, since we do not have $\mathrm{H}_{2}$ measurements from the beginning of our time series. Moreover, such a trend can be modified by a subsequent shock front. All this can be seen in Fig. 7, where three examples of the observed knot flux variability are shown. In addition more examples of flux variability are shown in Appendix C. The measured flux in the different filters ([S II] - circles, $\mathrm{H} \alpha$ - triangles, and $\mathrm{H}_{2}$ - squares) is plotted as a function of time. The top panel of Fig. 7 shows the appearance in 1989 of knot HH 52 B5, which rapidly increased its brightness and then faded at optical wavelengths, while the $\mathrm{H}_{2}$ emission seems to rise later. Central and bottom panels of the figure illustrate two more examples of variability in $\mathrm{HH} 52$. HH $52 \mathrm{C} 1$ has a trend similar to B5, but the collision with a fast overtaking shock between 1995 and 2006 (see also Appendix C) newly increases the $\mathrm{H} \alpha$ and [S II] luminosity. Finally, knot HH 52 C2 (bottom panel) represents a quite rare case of [S II] variability, where the knot appears visible in $\mathrm{H} \alpha$ only in 2006.
In Fig. 8 an example of PM and flux variability is indicated. The bottom panel gives the PM values for the optical filters (no molecular emission is detected) of HH 52 B1 plotted against time. As described in Sect. 3.1.5. The PM value decreases in both the [S II] and $\mathrm{H} \alpha$ filters (see Table C.1, Col. 4), with a deceleration of $0.022 \pm 0.003$ and $0.02 \pm 0.01^{\prime \prime} \mathrm{yr}^{-2}$, respectively. We also note that the PAs of the linear motion and of the accelerated motion (Table C.1, Cols. 5 and 6) are identical in both species (within the errors). In addition the opposite direction of the accelerated motion indicates deceleration of the knot for both species. This is also visible in our linear fit of Fig. 8 (bottom panel), where we consider both species deriving an average deceleration value of $0.017 \pm 0.005^{\prime \prime} \mathrm{yr}^{-2}$ or $0.022 \pm 0.002^{\prime \prime} \mathrm{yr}^{-2}$ without considering the 1990 [S II] data point, apparently discordant. Also the flux of the knot varies with time, with a delay of about 3 years between the [S II] and $\mathrm{H} \alpha$ lines (as shown in Fig. 8, top panel).

In Fig. 9, as an example of the observed variability in velocity, direction, and flux, the behaviour of optical knots HH 54 G0 and $\mathrm{G}$ is presented. As for the previous knot, our analysis of the accelerated motions of these objects can be found in Table C.1. These knots are part of the HH 52 flow $\left(\mathrm{PA} \sim 55^{\circ}\right)$ in the NE region of the main body of HH54 (see also Figs. 4, A.2, and Appendices A and B), where the fast flow is suddenly decelerated as it collides with the leading material, which is partially deflected sideways $\left(\mathrm{PA} \sim 80^{\circ}\right)$. G0 is part of such a fast flow, that shocks at the end a slow moving component (knot G), changing its direction. Bottom panel of the upper figure indicates the measured PMs of G0 (circles) and G (triangles) at different epochs in 


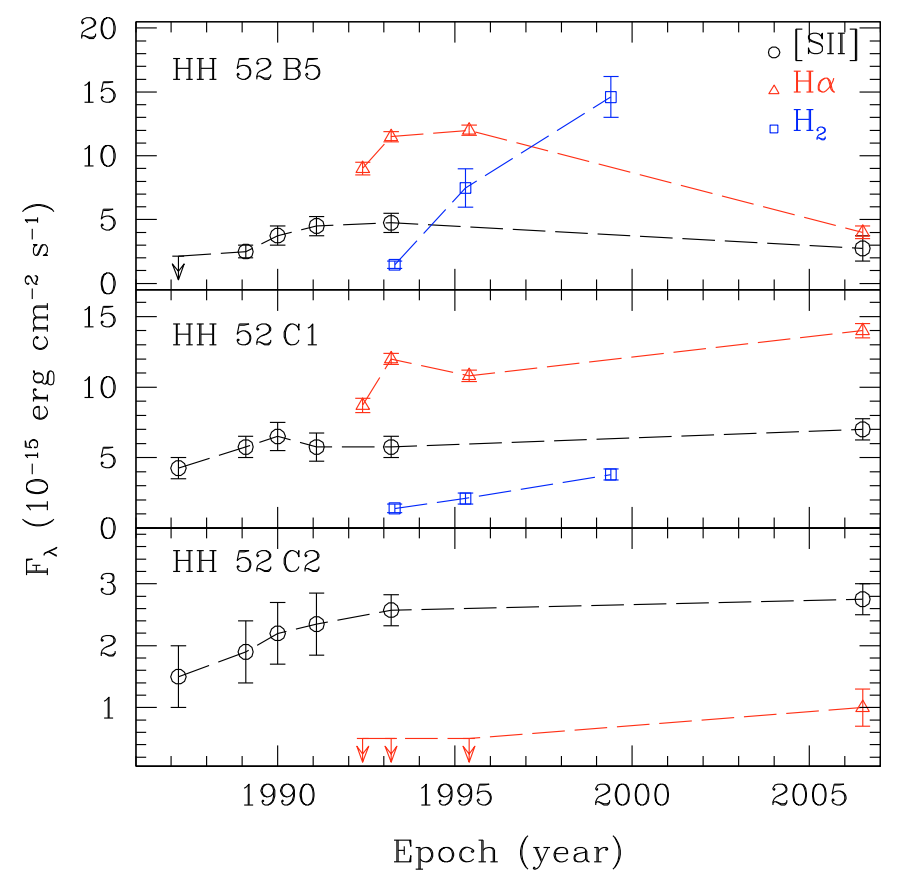

Fig. 7. Measured fluxes (uncorrected for the extinction) in knots HH 52 B5 (top panel), C1 (central panel), and C2 (bottom panel) in [S II] (circles), $\mathrm{H} \alpha$ (triangles), and $\mathrm{H}_{2}$ (squares) filters.

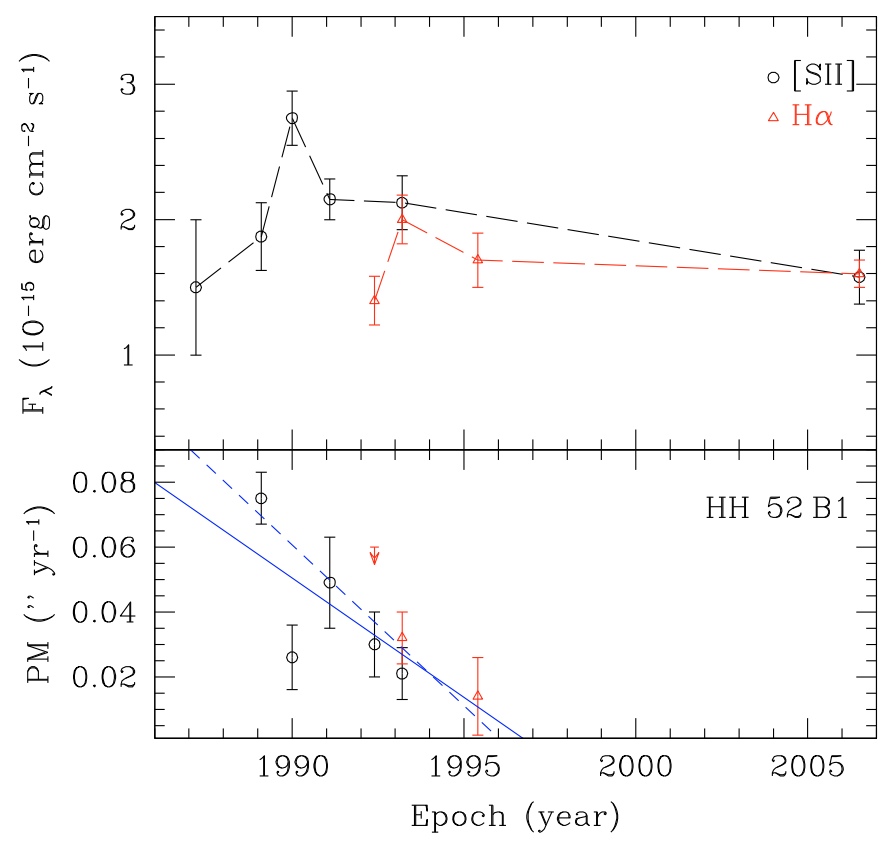

Fig. 8. Variability in HH 52 B1. Bottom panel shows the PMs as a function of time in both [S II] (circles), and $\mathrm{H} \alpha$ (triangles). The continuous and dashed lines are the best fits of the data points with and without 1990 [S II] point, respectively. The slope of the fit gives the deceleration of the knot. In the top panel the knot flux (in both filters) (uncorrected for the extinction) as a function of time is indicated.

both optical filters (open and filled marks are $\mathrm{H} \alpha$ and [S II] data, respectively). The top panel (upper figure) shows the variations of PAs with time for both knots in both filters. Finally, the lower figure reports the flux variability of the knots. The PM in G0 decreases from $\sim 0.07$ to $\sim 0.04^{\prime \prime} \mathrm{yr}^{-1}$ (i.e. from a $v_{\tan } \sim 65 \mathrm{~km} \mathrm{~s}^{-1}$ to
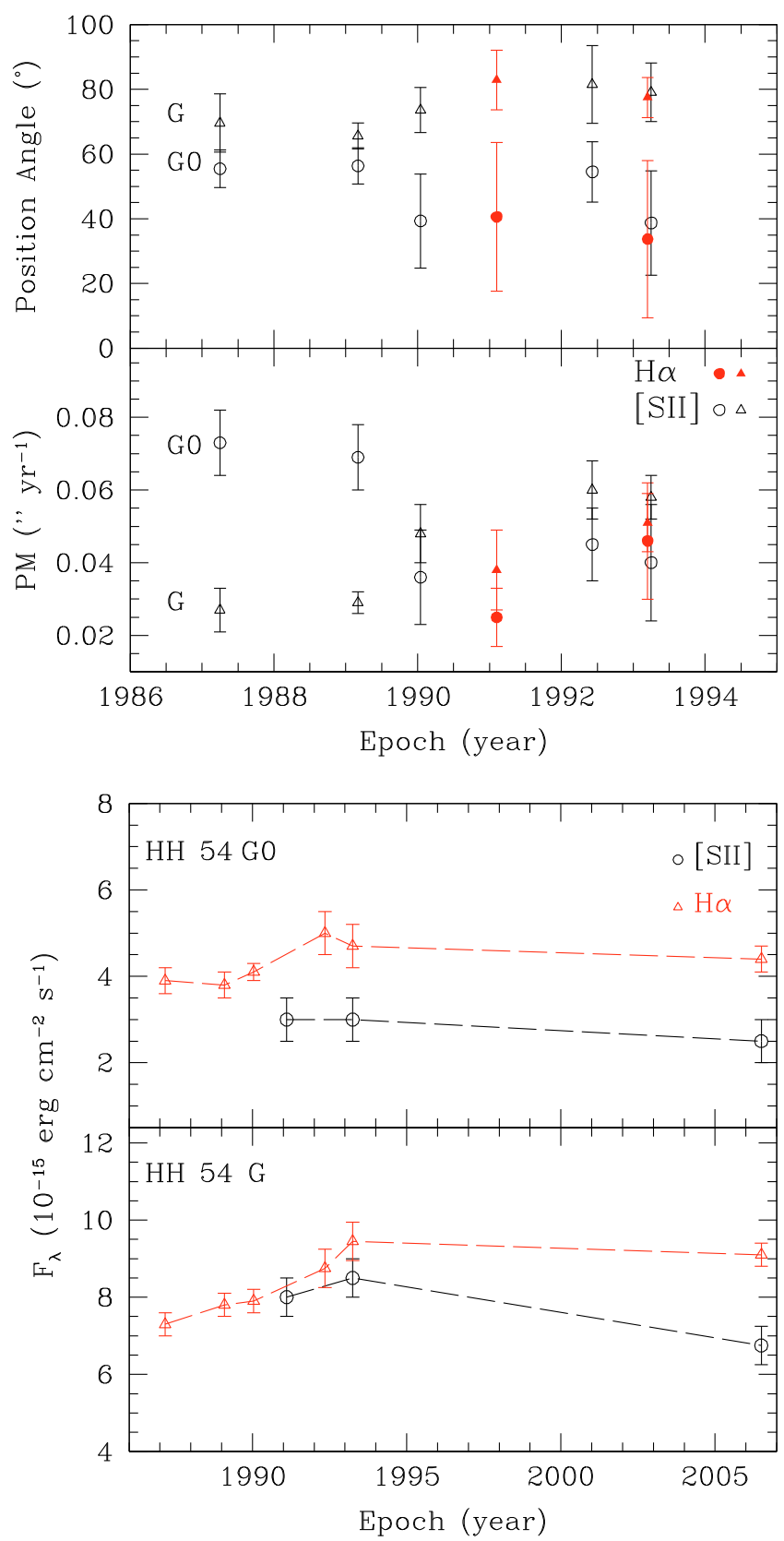

Fig. 9. Upper figure: changes of proper motions (bottom panel) and directions (top panel) in HH54 G (triangles) and G0 (circles) with time. Open and filled marks indicate measurements from $\mathrm{H} \alpha$ and [S II], respectively. Lower figure: measured (uncorrected for the extinction) fluxes in HH $54 \mathrm{G}$ (bottom panel) and G0 (top panel) in [S II] (circles), $\mathrm{H} \alpha$ (triangles), and $\mathrm{H}_{2}$ (squares) filters.

a $v_{\tan } \sim 35 \mathrm{~km} \mathrm{~s}^{-1}$ ) as it interacts with knot $\mathrm{G}$ ahead. At the same time, its PA also exhibits an abrupt change from $\sim 55^{\circ}$ to $\sim 39^{\circ}$. Accordingly, PM of knot G increases from $\sim 0.03$ to $\sim 0.06^{\prime \prime} \mathrm{yr}^{-1}$ (i.e. from $\sim 25$ to $\sim 53 \mathrm{~km} \mathrm{~s}^{-1}$ in $v_{\text {tan }}$ ), while its PA turns from $\sim 65^{\circ}$ to $\sim 80^{\circ}$. Indeed such a mechanism could at least partially explain the different PAs observed along the flows. As for the other knots, we observe a flux variation in both knots as well (Fig. 9, lower figure). 


\subsection{Radial velocities}

In Tables D.1-D. $4^{9}$, the results from our spectroscopical analysis are reported. From Tables D.1 to D.3, we list, for each object and optical instrument (EMMI and $\mathrm{B} \& \mathrm{C}$ ), the detected lines, the vacuum wavelength, radial velocities $\left(v_{\text {rad }}\right)$, and calibrated fluxes. Finally, Table D.4 indicates the [Fe II] $(1.64 \mu \mathrm{m})$ radial velocities along the ISAAC slits. No flux calibration is available for the NIR spectra. Each $v_{\text {rad }}$ has been computed with respect to the local standard of rest (LSR), assuming a cloud speed of $2 \mathrm{~km} \mathrm{~s}^{-1}$ (see Knee 1992). When detected, two different velocity components are reported.

At variance with B\&C, the EMMI data allowed us to study single substructures inside the HH objects, thanks to the higher spatial and spectral resolution, and often two distinct velocity components were detected. Moreover, errors in the EMMI spectra are considerably smaller than in the B\&C (4 and $20 \mathrm{~km} \mathrm{~s}^{-1}$, respectively). However, for those regions encompassed by slits of both instruments, there is excellent agreement among the values measured with the two instruments. It is also worth noting that all the measured velocities of the knots in this region are blueshifted, as mentioned by Graham \& Hartigan (1988). With a few exceptions, our results are in excellent agreement with theirs. Due to reduced resolution, however, both their spectra and our B\&C spectra only show one velocity component.

Our measured radial velocities range between -20 and $-110 \mathrm{~km} \mathrm{~s}^{-1}$ with variations similar to those detected in the PMs. The correlation between radial velocities and PMs implies that knot velocity variations are real. With a few exceptions, radial velocities derived from the detected (atomic) lines have similar values. The highest values (around $-110 \mathrm{~km} \mathrm{~s}^{-1}$ ) are observed in the three knots of the HH 53 flow (A-C) (see Table D.2) and along the two streamers (around $-100 \mathrm{~km} \mathrm{~s}^{-1}$ ). A change in the radial velocity (from $\sim 100 \mathrm{~km} \mathrm{~s}^{-1}$ up to $\sim 40 \mathrm{~km} \mathrm{~s}^{-1}$ ) is observed ahead of the HH 52 streamer in groups HH $54 \mathrm{G}$ and C, where the fast flow impacts slow-moving material.

The line-profile analysis of the two velocity components indicates that they originate from single bow-shock structures (as in HH $52 \mathrm{~A}$ ), from two different flows (as in HH $53 \mathrm{C}$ and $\mathrm{C} 1$ ), or from a flow at different speeds (e.g. in HH 54 G) (see details in Appendix D).

Figures 10 and 11 show the position velocity (PV) diagrams of the two ISAAC spectra. Since we do not have any [Fe II] image, we use an $\mathrm{H} \alpha$ image (EMMI 2006) to indicate the position of the slits, enlarged to match the spatial resolution of ISAAC. The accordance between the $\mathrm{H} \alpha$ and [Fe II] emissions is quite satisfactory. The observed velocity values range from about -10 to $-110 \mathrm{~km} \mathrm{~s}^{-1}$. The velocity structure of the region appears complex, and again, often two velocity components are detected. At variance with the EMMI spectra,it is more difficult here to tell whether these components originate from single bow shock structures or from the overlapping of two different flows (i.e. HH 52 and 54).

Sometimes the two velocities are well-separated, as in B3 or close to B (Fig. 10), or as in C1 and C3 (Fig. 11). For these objects, the superposition of the two different flows is very likely. The fast component would then originate from the HH 54 flow, which shows in $\mathrm{Z}$ a $v_{\text {rad }}$ of $\sim-100 \mathrm{~km} \mathrm{~s}^{-1}$ (see Fig. 10). In other cases, such as in A1, B, H2, and H3 along slit 1 (see Fig. 10), the two velocity components are not well-separated and the line profiles resemble those of a bow shock. For HH 54 B, however, the highest intensity in the spectral profile is detected in the lower

9 Tables D.1-D.4 are presented in Appendix D.

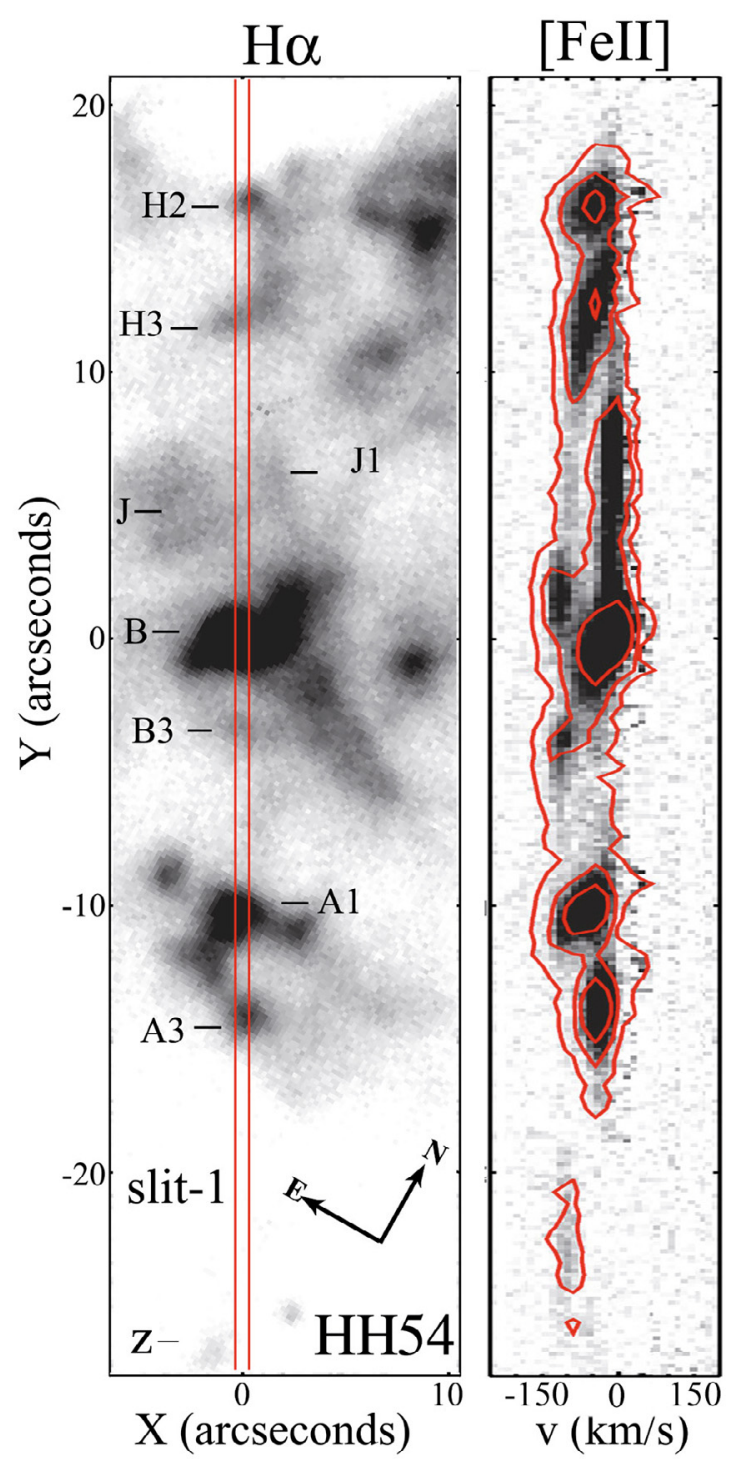

Fig. 10. $P-V$ diagram of $\mathrm{HH} 54$ slit 1 . In the left panel, an $\mathrm{H} \alpha$ image with the slit superimposed is reported. In the right the [Fe II] $(1.64 \mu \mathrm{m})$ spectrum is reported.

velocity component, as expected in a cloudlet geometry (see, e.g., Schwartz 1978) and not in the higher velocity component, as observed in a bullet model (e.g. Hartigan et al. 1987; Davis et al. 2001). In the first case the observed PMs should be close to zero, and, indeed, in knot B we observe a $v_{\tan }$ close to zero.

\subsection{Inclination and spatial velocity}

By combining tangential and radial velocities it is possible to infer an upper limit of the inclination of the single knots and flows (see e.g. 1994b). In order to calculate the inclination of each structure and to reduce the uncertainties, we have inferred from our optical data an average value of both the tangential and radial velocities. In Table E. $1^{10}$ the inclination and spatial velocity derived for each knot are reported (averaging both $\mathrm{H} \alpha$ and $[\mathrm{S} \mathrm{II}]$ values). A detailed analysis is given in Appendix E. Inferred spatial velocities range from 25 to $125 \mathrm{~km} \mathrm{~s}^{-1}$. We obtained three different averaged values of inclination for the flows $\left(58 \pm 3^{\circ}, 84 \pm 2^{\circ}\right.$, and $67 \pm 3^{\circ}$ for HH 52, 53, and 54 flows,

10 Table E. 1 is presented in Appendix E. 


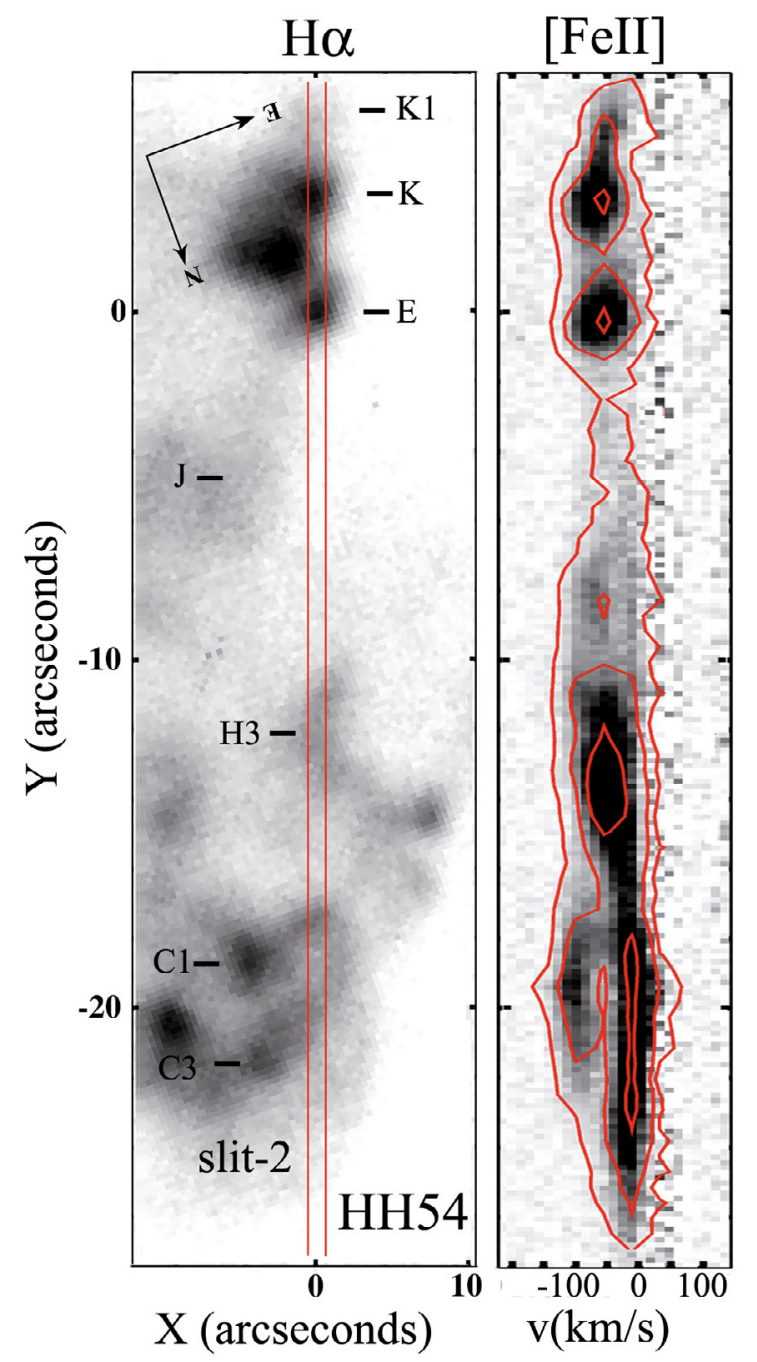

Fig. 11. $P-V$ diagram of HH 54 slit 2. In the left panel an $\mathrm{H} \alpha$ image with the slit superimposed is reported. In the right the [Fe II] $(1.64 \mu \mathrm{m})$ spectrum is reported.

respectively), which support the proposed scenario of three distinct outflows. The outcome is illustrated in Fig. 12, where the measured knots are spatially plotted with their PMs, PAs, and inclinations (different colours represent different range of inclinations with respect to the plane of the sky, a few more knots of HH 54 have been included combining [Fe II] and optical data see next paragraph).

We also attempted to estimate the inclination angle for the structures encompassed by the [Fe II] ISAAC slits, in order to define their proper outflow. To this aim we tentatively combined the $[\mathrm{Fe}$ II] radial velocities with the atomic tangential velocities derived from the optical lines, keeping in mind that these species can trace different velocities and regions of the shock. The results are reported in Fig. 12 as well. For knot Z in the HH 54 streamer we obtain an inclination angle of $72^{\circ} \pm 2^{\circ}$, in good agreement with the optical results. Both $\mathrm{K}$ and $\mathrm{E}\left(i=73^{\circ} \pm 2^{\circ}\right)$ are also part of this flow. Knot A1 could be part of the HH 52 flow $\left(i=62^{\circ} \pm 4^{\circ}\right)$, while the association of knot A3 is not clear $\left(i=68^{\circ} \pm 8^{\circ}\right)$. Also $\mathrm{J}, \mathrm{H} 2$, and $\mathrm{H} 3$ are likely parts of this flow with the inclination estimates of $58^{\circ} \pm 6^{\circ}, 54^{\circ} \pm 5^{\circ}$, and $53^{\circ} \pm$ $3^{\circ}$, respectively.

\subsection{Diagnostic results from spectroscopy}

From the ratio of the detected lines of our optical spectra, it is also possible to derive the physical parameters of the encompassed knots. The selected optical transitions from [O I], [N II], and [S II] were used to determine the electron density $\left(N_{\mathrm{e}}\right)$, electron temperature $\left(T_{\mathrm{e}}\right)$, and ionisation fraction $\left(X_{\mathrm{e}}\right)$ of the shocked gas by adopting the so-called BE technique (e.g. BE99, Nisini et al. 2005; Podio et al. 2006), which in turn allows us to derive the total hydrogen density $N_{\mathrm{H}}\left(N_{\mathrm{e}} / X_{\mathrm{e}}\right)$ as well. The assumed chemical abundances are those from Esteban et al. (2004), extensively discussed and also used in Podio et al. (2006). The results are presented in Table 3 (Cols. 2-5). The observed lines have been dereddened using the optical extinction law of Cardelli et al. (1987) assuming an average $A_{\mathrm{V}}$ value of $2 \pm 1$ mag for all the objects, as retrieved in previous works on HH 54 from the NIR analysis (Gredel 1994; Caratti o Garatti et al. 2006; Giannini et al. 2006). The visual extinction in HH 52 and 53 should have similar values as the extinction map in Porras et al. (2007) suggests (see their Fig. 9). The errors of the physical parameters in Table 3 were derived by combining the uncertainties on both the line fluxes and extinction.

The values appear quite homogeneous along and among the three flows, indicating the presence of very light and ionised jets, as sometimes observed in other HH flows, where shocks propagate in a medium of relatively low pre-shock density (see e.g. Podio et al. 2006). Actually, both ionisation fraction and electron temperature have high values, ranging between $0.2-0.6$ and 14000-26000 K, respectively. On the other hand, both hydrogen and electron densities are extremely low with values of $2 \times 10^{2}-2 \times 10^{3} \mathrm{~cm}^{-3}$ and $50-790 \mathrm{~cm}^{-3}$, respectively. Remarkably, such a low jet density for HH 54 was predicted by the shock models of Giannini et al. (2006), supported by $\mathrm{H}_{2}$ and FIR observations. There, the emission in HH 54 was interpreted as originated by a J-type shock with a magnetic precursor of $N_{\mathrm{H}} \sim 10^{3} \mathrm{~cm}^{-3}$, which interacts with a relatively denser ambient medium $\left(N_{\mathrm{H}} \sim 10^{4} \mathrm{~cm}^{-3}\right)$.

\subsection{Mass flux, mass, and luminosity}

The physical and kinematical parameters derived in the previous sections allow us to evaluate the mass flux rate of the knots $(\dot{M})$ in the studied $\mathrm{HH}$ objects. In particular, together with the direction retrieved from the PM analysis, $\dot{M}$ can help us to identify which exciting source is driving the outflow, comparing the mass flux rates of the outflow with the theoretical ejection rate of the candidate sources (also taking their evolutionary stage and bolometric luminosity into account).

We used two different methods to infer $\dot{M}$ (for a detailed discussion see Nisini et al. 2005; Podio et al. 2006). In the first method (A), the mass flux is estimated as: $\dot{M}_{\mathrm{k}}=\mu m_{\mathrm{H}} N_{\mathrm{H}} \pi r_{\mathrm{k}}^{2} v_{\mathrm{k}}$, where $\mu$ is the average atomic weight, $m_{\mathrm{H}}$ is the proton mass, $N_{\mathrm{H}}$ the hydrogen density, and $v_{\mathrm{k}}$ and $r_{\mathrm{k}}$ are the velocity and radius of the knot, respectively. For this we have taken half of the length of the knot, derived from a $3 \sigma$ contour on the images. Method A assumes that the knot is uniformly filled at the derived density, providing us with an upper limit of the mass flux. The second method (B) makes use of the luminosities of the optical lines ([S II], [O I], and [N II] doublets), the hydrogen density, and the tangential velocity: $\dot{M}_{\mathrm{k}}=\mu m_{\mathrm{H}}\left(N_{\mathrm{H}} V\right) v_{\mathrm{t}} / l_{\mathrm{t}}$, with $N_{\mathrm{H}} V=L_{\mathrm{X}}\left(h v A_{\mathrm{i}} f_{\mathrm{i}} \frac{\mathrm{X}^{\mathrm{i}}}{\mathrm{X}} \frac{[\mathrm{X}]}{[\mathrm{H}]}\right)^{-1}$, where $L_{\mathrm{X}}$ is the luminosity of the element $\mathrm{X}$, for the selected transition, $A_{\mathrm{i}}$ and $f_{\mathrm{i}}$ are the radiative rate and the fractional population of the upper level of the transition, 


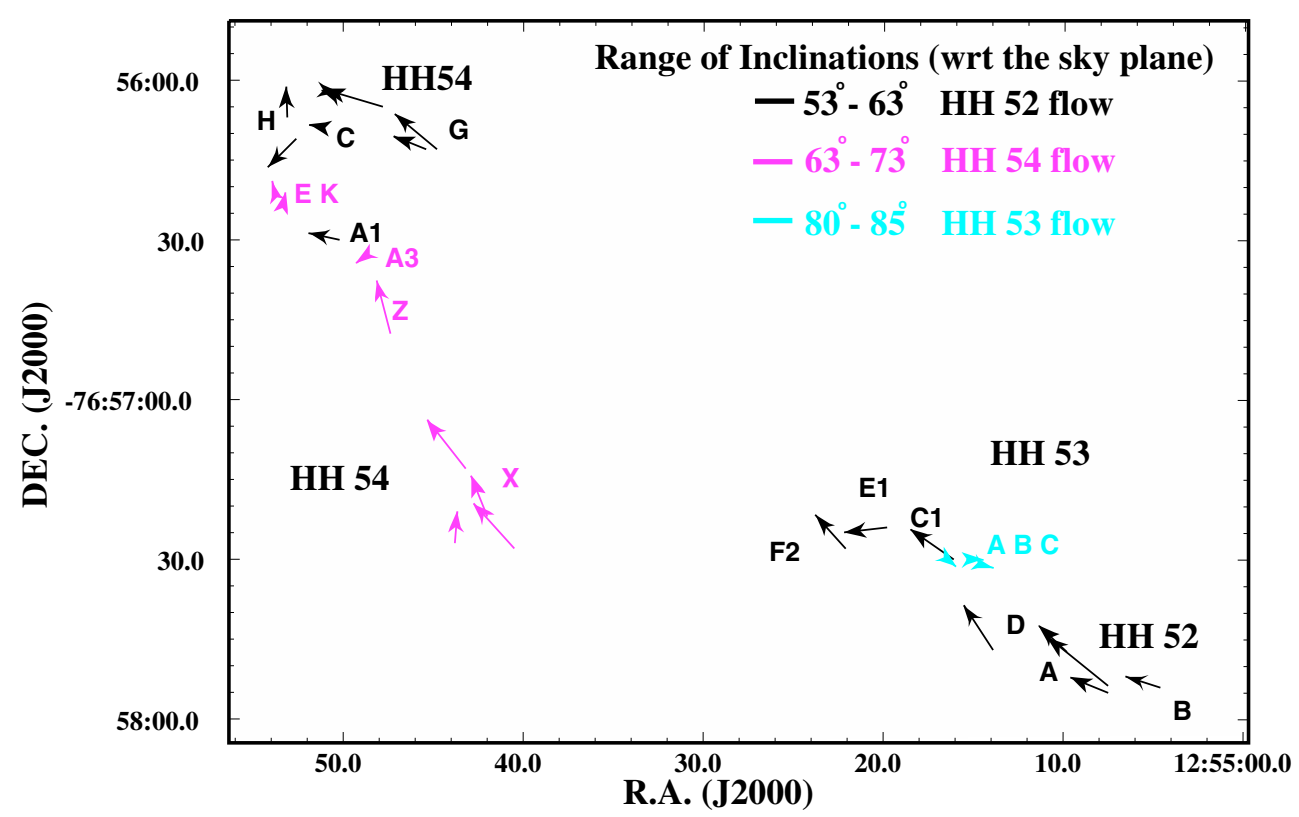

Fig. 12. Inclination of the flows with respect to the plane of the sky. The arrows show the direction of the knots. Their different colours indicate different range of inclination values with respect to the sky plane.

Table 3. Measured physical parameters and mass fluxes of knots in HH 52, HH 53, and HH 54.

\begin{tabular}{|c|c|c|c|c|c|c|c|}
\hline Knot & $\begin{array}{l}\log \left(N_{\mathrm{e}}\right) \\
\left(\mathrm{cm}^{-3}\right)\end{array}$ & $X_{\mathrm{e}}\left(N_{\mathrm{e}} / N_{\mathrm{H}}\right)$ & $\begin{array}{l}T_{\mathrm{e}} \\
(\mathrm{K})\end{array}$ & $\begin{array}{l}\log \left(N_{\mathrm{H}}\right) \\
\left(\mathrm{cm}^{-3}\right)\end{array}$ & $\begin{array}{l}r_{\text {knot }} \\
\left({ }^{\prime \prime}\right)\end{array}$ & $\begin{array}{l}\dot{M}(\mathrm{~A})^{a} \\
\left(10^{-8} M_{\odot} \mathrm{yr}^{-1}\right)\end{array}$ & $\begin{array}{l}\dot{M}(\mathrm{~B})^{b} \\
\left(10^{-8} M_{\odot} \mathrm{yr}^{-1}\right)\end{array}$ \\
\hline HH 52 A1 & $2.82 \pm 0.02$ & $0.43 \pm 0.02$ & $17500 \pm 700$ & $3.18 \pm 0.16$ & 1.5 & $8.2 \pm 0.7$ & $1.1 \pm 0.1$ \\
\hline HH 52 A $3-4$ & $2.46 \pm_{0.36}^{0.26}$ & $0.35 \pm 0.06$ & $23800 \pm 2600$ & $2.9 \pm 0.5$ & 1.3 & $1.5 \pm 0.4$ & $0.3 \pm 0.03$ \\
\hline HH 52 D4 & $2.2 \pm 0.2$ & $0.5 \pm 0.05$ & $21000 \pm 1000$ & $2.5 \pm 0.1$ & 1.6 & $2.1 \pm 0.2$ & $0.2 \pm 0.03$ \\
\hline HН 52 B & $2.88 \pm 0.03$ & $0.40 \pm 0.03$ & $21500 \pm_{800}^{900}$ & $3.28 \pm_{0.25}^{0.26}$ & 3.3 & $16 \pm 2$ & $3 \pm 0.3$ \\
\hline HH 52 D2-D3 & $2.45 \pm_{0.21}^{0.18}$ & $0.23 \pm_{0.06}^{0.04}$ & $14200 \pm_{1800}^{2400}$ & $3.09 \pm_{0.76}^{0.53}$ & 2.4 & $7 \pm 2$ & $1 \pm 0.1$ \\
\hline HH 53 A & $2.88 \pm 0.03$ & $0.43 \pm 0.03$ & $21600 \pm 800$ & $3.15 \pm 0.20$ & 0.8 & $1.1 \pm 0.2$ & $0.2 \pm 0.02$ \\
\hline HH 53 B & $2.60 \pm_{0.22}^{0.18}$ & $0.31 \pm_{0.09}^{0.05}$ & $17300 \pm_{1900}^{2500}$ & $2.92 \pm 0.03$ & 0.8 & $0.7 \pm 0.1$ & $0.3 \pm 0.02$ \\
\hline HH 53 C & $2.46 \pm 0.11$ & $0.34 \pm 0.04$ & $18600 \pm_{1100}^{1200}$ & $2.9 \pm 0.3$ & 0.6 & $0.3 \pm 0.03$ & $0.07 \pm 0.01$ \\
\hline HH $53 \mathrm{C} 1$ & $2.59 \pm_{0.15}^{0.13}$ & $0.32 \pm 0.09$ & $24200 \pm 3700$ & $3.08 \pm_{0.89}^{0.85}$ & 0.6 & $1.3 \pm 0.1$ & $0.3 \pm 0.06$ \\
\hline HH 54 C2 & $2.76 \pm_{0.05}^{0.06}$ & $0.49 \pm_{0.04}^{0.03}$ & $22600 \pm_{1600}^{2800}$ & $3.06 \pm 0.20$ & 0.7 & $0.3 \pm 0.05$ & $0.3 \pm 0.05$ \\
\hline HH 54 C 3 & $2.66 \pm_{0.16}^{0.15}$ & $0.50 \pm 0.07$ & $26000 \pm 7000$ & $2.9 \pm 0.4$ & 0.7 & $0.2 \pm 0.05$ & $0.1 \pm 0.05$ \\
\hline HH 54 G-G0 & $2.65 \pm 0.01$ & $0.6 \pm_{0.1}^{0.05}$ & $19700 \pm_{1700}^{800}$ & $2.85 \pm_{0.01}^{0.09}$ & 0.7 & $0.6 \pm 0.03$ & $0.05 \pm 0.01$ \\
\hline HH 54 G1-G3 & $2.00 \pm_{0.27}^{0.21}$ & $0.35 \pm_{0.11}^{0.09}$ & $23000 \pm 3000$ & $2.15 \pm_{0.65}^{0.56}$ & 0.7 & $19 \pm 6$ & $3 \pm 0.5$ \\
\hline HH 54 X3 & $1.70 \pm 0.7$ & $0.34 \pm 0.07$ & $25400 \pm_{3800}^{3600}$ & $2.17 \pm_{0.48}^{0.51}$ & 1.3 & $0.3 \pm 0.08$ & $0.4 \pm 0.2$ \\
\hline НH 54 X4A-X4B & $2.6 \pm 0.3$ & $0.45 \pm 0.05$ & $26000 \pm 2000$ & $3.0 \pm 0.2$ & 2.1 & $0.8 \pm 0.1$ & $0.6 \pm 0.2$ \\
\hline
\end{tabular}

Notes: ${ }^{a} \dot{M}$ derived with method A (see text) $\dot{ }^{b} \dot{M}$ measured from [S II], [O I], and [N II] line luminosities (average value) multiplied by a correction factor that takes the entire area of the knot into account (method B, see text).

$\frac{X^{i}}{X}$ is the ionisation fraction of the considered species with a total abundance of $\frac{[\mathrm{X}]}{[\mathrm{H}]}$ with respect to the hydrogen. Line fluxes were dereddened according to Sect. 4.7. The results from each doublet in each knot were averaged, obtaining a final value. The values of the mass flux derived from this method can be considered as lower limits (e.g. Nisini et al. 2005; Podio et al. 2006), because method B only measures gas that is sufficiently heated to radiate in the observed lines. A correction factor was applied when the slit width was less than the encompassed knot. The [S II] images allowed us to derive such a quantity, however, for the other two atomic species. The dimension of the emitting region could not be inferred, and the same correction factor has been adopted, as well. In Table 3 (Cols. 7-8) the values of the mass flux derived from both methods are reported. The resulting mass fluxes are presented in Fig. 13, as well, where for each flow, $\dot{M}$ of each group of knots is plotted as a function of the distance from the first knot of the flow. As expected $\dot{M}(A)$ is, on average, almost an order of magnitude greater than $\dot{M}(B)$ (in 2 out of 3 flows). Along the HH 52 flow (bottom panel of Fig. 13), $\dot{M}(A)$ oscillates between $\sim 2 \times 10^{-7}$ and $\sim 10^{-8} M_{\odot} \mathrm{yr}^{-1}$ (between $\sim 3 \times 10^{-8}$ and $\sim 3 \times 10^{-9} M_{\odot} \mathrm{yr}^{-1}$ with method B). Mass flux along HH 53 flow $\left(M_{\odot}(A) \leq 10^{-8} M_{\odot} \mathrm{yr}^{-1}, M_{\odot}(B) \leq 3 \times 10^{-9} M_{\odot} \mathrm{yr}^{-1}\right)$ is more than an order of magnitude lower than in HH 52. This could indicate a less massive and/or an older exciting source. The value 

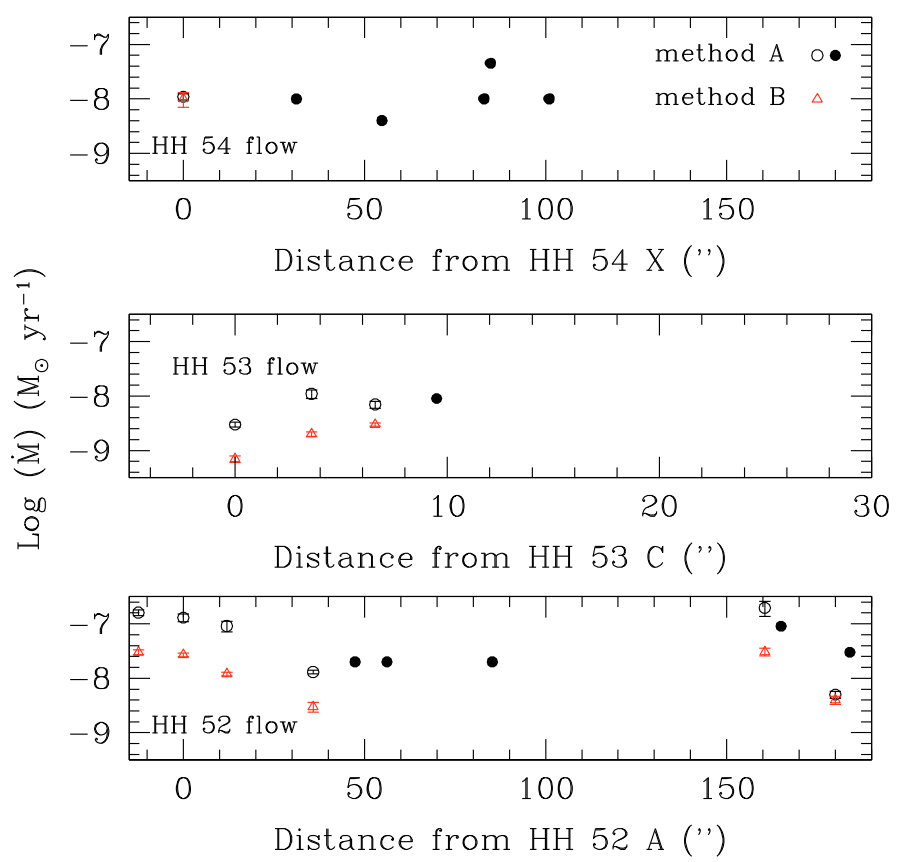

Fig. 13. Mass fluxes derived for HH 52 (bottom panel), HH 53 (central panel), and HH 54 flows (upper panel). Circles and triangles refer to $\dot{M}$ values inferred from method A and B, respectively. Filled circles refer to $\dot{M}$ estimates obtained with method A, assuming an $N_{\mathrm{H}}$ average value of $10^{3} \mathrm{~cm}^{-3}$.

in HH $54 \mathrm{X}$ (along the streamer) is almost the same with both methods $\left(\sim 10^{-8} M_{\odot} \mathrm{yr}^{-1}\right)$.

Using method $\mathrm{A}$ and considering an average hydrogen density of $10^{3} \mathrm{~cm}^{-3}$ (see also Table 3 ), we also estimated the mass flux for most of the other knot groups. The values are also reported in Fig. 13 as filled circles. $\dot{M}(A)$ has almost the same value in $\mathrm{HH} 52 \mathrm{E}, \mathrm{F}$, and $\mathrm{G}\left(\sim 2 \times 10^{-8} M_{\odot} \mathrm{yr}^{-1}\right)$ Along the HH 54 streamer, the estimate of $\dot{M}(A)$ ranges between $3 \times 10^{-9}$ and $\sim 10^{-8} M_{\odot} \mathrm{yr}^{-1}$ in $\mathrm{Y}$ and $\mathrm{Z}$, respectively. On the main body of $\mathrm{HH} 54$, we can evaluate a mass flux rate of about $9 \times, 4.5 \times$, $3 \times, 1 \times 10^{-8} M_{\odot} \mathrm{yr}^{-1}$ for A, E-K, H, and I, respectively.

It is worth noting that such values are in excellent agreement with the mass flux rates estimated in Giannini et al. (2006) and with the linear momentum $(\dot{P})$ obtained from CO in Knee (1992), considering the velocities and the mass flux rates measured in this paper. This agreement between atomic and molecular momentum flux can only be explained if the jet is strongly under-dense with respect to the ambient medium (10-100 times). Then the efficiency of the momentum transfer from the jet $(\mathrm{HH})$ to the medium $(\mathrm{CO})$ is close to one.

Moreover, to measure the energy budget of the flows, we computed mass and luminosity of the knots (for the detected species, i.e. HI, [S II], and $\mathrm{H}_{2}$ ). To derive the mass, we assumed $M_{\mathrm{k}}=\mu m_{\mathrm{H}} N_{\mathrm{H}} V$, where $\mu$ is the average atomic weight, $m_{\mathrm{H}}$ is the proton mass, $N_{\mathrm{H}}$ the hydrogen density, and $V$ the volume of the knot. For those knots where the hydrogen density was not measured, we assumed an $N_{\mathrm{H}}$ average value of $10^{3} \mathrm{~cm}^{-3}$ (see also Table 3). The luminosity of each element is $L=4 \pi d^{2} I$, where $d$ is the distance and $I$ the total intensity. The total intensity is derived from the dereddened line fluxes measured from narrowband imaging. As in Sect. 4.7 the adopted extinction value is $A_{\mathrm{V}}=2 \pm 1 \mathrm{mag}$. For the [S II] emission an NLTE model has been employed to derive the line ratio with respect to the sulphur doublet (see e.g. Nisini et al. 2005; Caratti o Garatti et al. 2006). The input parameters are $T_{\mathrm{e}}, N_{\mathrm{e}}, X_{\mathrm{e}}$, and $A_{\mathrm{V}}$. Where $T_{\mathrm{e}}, N_{\mathrm{e}}, X_{\mathrm{e}}$ have not been measured, average values of $20000 \mathrm{~K}, 10^{-3} \mathrm{~cm}^{-3}$, and 0.4 , have been adopted, respectively. The HI intensity was obtained from the $\mathrm{H} \alpha$ line, assuming that the radiated energy is emitted under Case B recombination, and using emissivity values from Storey \& Hummer (1995). Finally, for the molecular hydrogen, when observed, we consider an LTE gas at $T=$ 2000 K (see e.g. Caratti o Garatti et al. 2006).

\subsection{The candidate driving sources}

As seen in Sect. 4.1, our $\mathrm{H}_{2}$ maps do not reveal any molecular jet close to the possible driving sources, therefore we do not have any direct indication of which are the driving sources of these flows. We thus selected the possible candidates on the basis of several YSO catalogues (i.e. IRAS, $2 M A S S$, and MSX) and publications (see e.g. Young et al. 2005; Spezzi et al. 2007; Alcalá et al. 2008; Spezzi et al. 2008), on the IR colours of the YSO in the Spitzer images, and on their positions with respect to the HH objects. In Fig. 2, the $24 \mu \mathrm{m}$ Spitzer-MIPS map of the region indicates the selected candidates and the location of the studied $\mathrm{HH}$ objects. There are five IRAS sources in the region with a position barely compatible with the derived PA of the HH flows. These young sources have been deeply analysed in the literature (see i.e. Spezzi et al. 2007; Alcalá et al. 2008; Spezzi et al. 2008), where the authors report their main characteristics and spectral energy distributions (SEDs). From the Spitzer IR colours, we recognised two more possible candidates that have been added to our list. We note, however, that these two objects were not included in previous Spitzer studies of the region, because they do not fit some of the selection criteria used (see Spezzi et al. 2007). In particular the SED analysis in the C2D catalogue indicates that Source 1 (see Fig. 2) could be a galaxy. The sources, together with short comments, are reported below. The SEDs of the eligible candidates are shown in Fig. 14. They have been obtained combining $2 M A S S$, Spitzer, IRAS, and literature data.

IRAS 12496-7650 (DK Cha) is a Herbig Ae star with a high velocity and weak CO outflow (PA $\sim 10^{\circ}-15^{\circ}$, Knee 1992), apparently not related with HH 52, 53, or 54 outflows (Knee 1992), even if its estimated mass accretion rate $\left(\dot{M}_{\mathrm{acc}} \sim 10^{-6} M_{\odot} \mathrm{yr}^{-1}\right.$, Alcalá et al. 2008) would make it an excellent candidate (see discussion in Alcalá et al. 2008). Our PM measurements of the three flows indicate, however, that this cannot be the driving source of any of these flows.

IRAS $12448-7650$ is a Class III YSO with a $\dot{M}_{\text {acc }} \sim$ $10^{-8}-10^{-9} M_{\odot} \mathrm{yr}^{-1}$ (Alcalá et al. 2008) and located $\sim 68^{\circ}$ with respect to $\mathrm{HH} 52$ flow, i.e. $10^{\circ}-15^{\circ}$ of misalignment. Moreover, its optical spectrum (Alcalá and Spezzi private communication) shows no Li I absorption feature, indicating that the source has an age $\geq 2 \times 10^{6} \mathrm{yr}$ (see e.g. Magazzú et al. 1992).

IRAS 12416-7703, a Class II YSO (Young et al. 2005; Alcalá et al. 2008), has the correct position angle $\left(\sim 56^{\circ}\right)$ to drive the HH 52 flow. The SED analysis reveals that the source has a bolometric luminosity of about $8 L_{\odot}$ (see Fig. 14) (a similar value is by Spezzi et al. 2008). In theory, this would be the most reliable candidate for the HH 52 flow. Nonetheless, its optical spectrum (Alcalá and Spezzi private communication) has a weak $\mathrm{H} \alpha$ emission but no Li I absorption feature, thus IRAS 12416-7703 seems too old to be the driving source. It is puzzling, however, that no other YSOs are present in this region (see Figs. 2 and 9 in Alcalá et al. 2008).

IRAS 12500-7658 is a Class I (Chen et al. 1997) source with an $L_{\text {bol }}$ of $0.5 L_{\odot}$ (Young et al. 2005) with an estimated $\dot{M}_{\text {acc }}$ of $2 \times 10^{-6} M_{\odot} \mathrm{yr}^{-1}$ (Alcalá et al. 2008). Indeed this is the best candidate for the HH 54 flow $\left(\dot{M} \sim 10^{-8} M_{\odot} \mathrm{yr}^{-1}\right.$, see Fig. 13) 


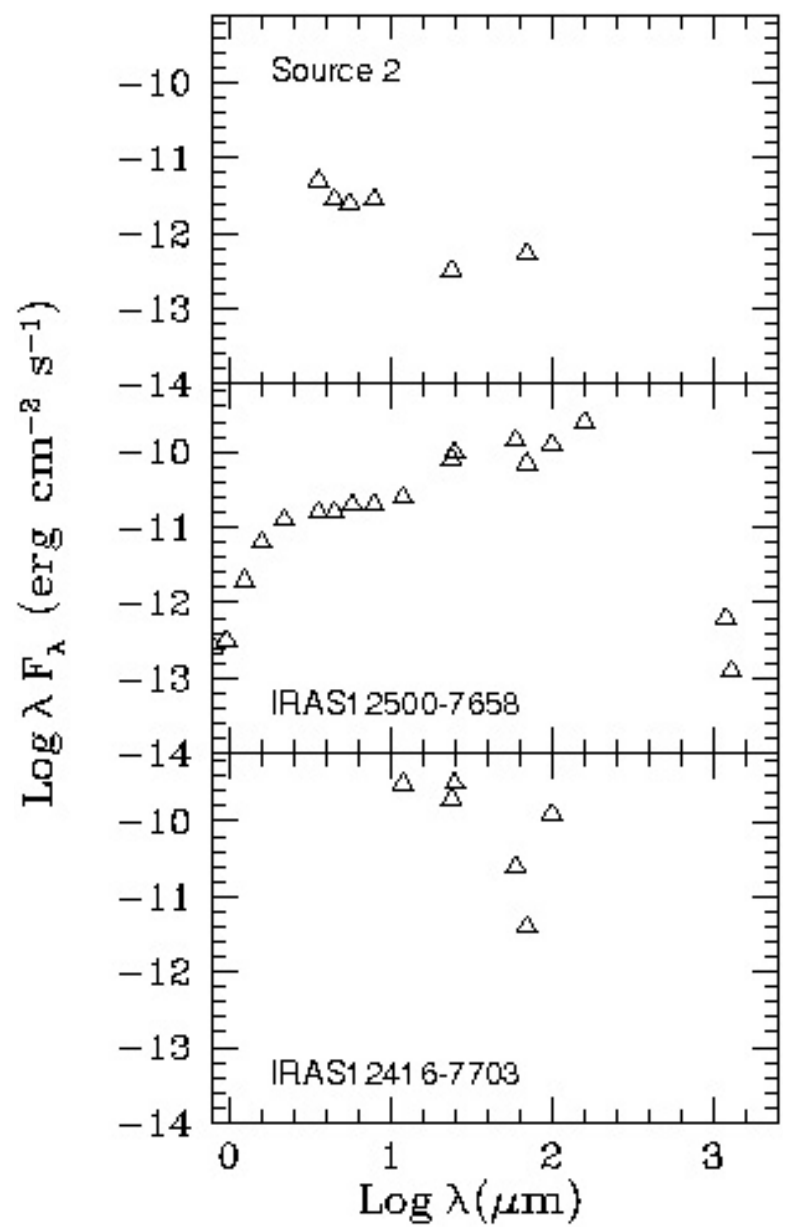

Fig. 14. SEDs of the discussed candidate driving sources.

because of the PA of $\sim 20^{\circ}$ with respect to the $\mathrm{HH}$ object and the $\dot{M} / \dot{M}_{\text {acc }}(0.1-0.01)$ ratio, compatible with a Class I source.

IRAS F12488-7658/C13, located two arcminutes west of the previous source, is a Class III YSO (Voung et al. 2001) with a $\dot{M}_{\text {acc }} 4 \times 10^{-8} \mathrm{yr}^{-1}$ (Alcalá et al. 2008) and has a PA $\sim 30^{\circ}$ with respect to HH 54. The misalignment, the age, and the $\dot{M}_{\text {acc }}$ of the source seem to indicate that this is not the driving source.

Finally, we detect in the Spitzer images an MIR source almost coincident with HH 53 (indicated as Source 2 in Fig. 2, $\alpha=12^{\mathrm{h}} 55^{\mathrm{m}} 17^{\mathrm{s}}$ and $\delta=-76^{\circ} 57^{\prime} 29^{\prime} \cdot 3$ ), appearing as a faint star in our optical, $\mathrm{H}_{2}$, and $2 M A S S$ images. A faint emission is also visible in the IRAC images (partially detached with respect to the HH 53 knots), while this object is not resolved in the MIPS images and part or all the measured flux could originate in the $\mathrm{HH}$ itself. The coordinates of this object are almost the same as the infrared source $\mathrm{HH} 53^{*} 1$ reported by Sandell et al. (1987) and indicated as the possible driving source of HH 53. On the contrary, Graham \& Hartigan (1988) firmly rejected this hypothesis because their observations showed a blueshifted emission both to the west and to the east of the star (we know, however, that such emission could originate in the HH 52 flow) and because it did not show any unusual strong emission feature in the star spectrum. Furthermore, the optical photometry of Spezzi and Alcalá (private communication) indicates that the object would not belong to Cha II and could be a background star. Nonetheless, our SED analysis (Fig. 14, from the Spitzer photometry and under the uncertain hypothesis that the MIR comes entirely from the object) would indicate that it is a Class II YSO, with a $L_{\text {bol }} \sim 10^{-2} L_{\odot}$. We tentatively speculate that this could be the exciting source of HH 53. The mass ejection rate measured on the knots $\left(3 \times 10^{-9}-10^{-8} M_{\odot} \mathrm{yr}^{-1}\right)$ appears, however, too high compared to the Class and the luminosity of the presumed source. Indeed a more in-depth spectroscopical analysis is needed to solve this conundrum. Nevertheless, it is worth noting that no other candidates are detected east of HH 53. In conclusion, two of the three identified flows (i.e. HH 52 and 53) still have not a reliable driving source candidate.

\section{Discussion}

\subsection{Variability}

\subsubsection{Flux and velocity variability of knots}

Our analysis indicates that flux and velocity variability is observed in knots along the flows mostly in coincidence with interacting knots or working surfaces, where pairs of knots interact by accelerating and decelerating the gas in front and behind, respectively, or where the jet/streamer hits the slow moving material ahead. On the other hand, knots with a constant or slightly variable flux do not show relevant changes in velocity. It is therefore reasonable to investigate if and how such a flux variability is connected to the observed velocity variations. We can argue that part of the kinetic energy of the impacting knot is transferred to the preceding knot or to the surrounding medium, and then converted in radiated energy. However, it is worth noting that here we do not consider how the energy is transformed and which processes are involved. In a forthcoming paper (De Colle $\&$ Caratti o Garatti, in prep.), we report how the observed variability can be reproduced by MHD simulations, when knots interact along the flow.

In order to check how much of the kinetic energy is radiated during this process, we therefore computed the energy budget for those knots that are interacting and that clearly show both flux increment and velocity decrement. We estimate both the amount of kinetic energy lost in the interaction $(\Delta K)$ and the variation in the radiated energy during the time interval of our observations $\left(\Delta E_{\mathrm{rad}}\right)$. The kinetic energy lost by the knot can be expressed as

$\Delta K_{\mathrm{k}}=1 / 2 M_{\mathrm{k}} \Delta v^{2}$

where $M_{\mathrm{k}}$ is the mass of the knot and $\Delta v^{2}$ is the difference between the initial and final squared velocities.

On the other hand, $\Delta E_{\mathrm{rad}}$ can be written as,

$\Delta E_{\mathrm{rad}}=\sum_{\text {species }}\left(\int_{t 1}^{t 2} \Delta L \mathrm{~d} t\right)$,

which is the integral of the luminosity variations over the time interval of observations, summed for all the detected species (i.e. HI, [S II], and $\mathrm{H}_{2}$ ). Knot luminosities, and masses were estimated as described in Sect. 4.8. The results are reported in Table 4 , where $\Delta E_{\text {rad }}$ of each element, and the total $\Delta E_{\text {rad }}$ are indicated for each analysed knot $\Delta K$.

As a result $\Delta E_{\text {rad }}$ is always smaller than $\Delta K$. This is possibly due to the longer cooling time of the gas with respect to the interval of our observations. Moreover this is also because we are not taking into account the $\Delta E_{\text {rad }}$ contribution from other species like FeII, OI, or CO, for example. We can give a rough upper limit estimate of the FeII contribution, if any, for example. The brightest [Fe II] emission in HH 52-54 complex is observed in HH54 B, where a flux of $\sim 8 \times 10^{-15} \mathrm{erg} \mathrm{s}^{-1} \mathrm{~cm}^{-2}$ (1.64 $\mu \mathrm{m}$ line) has been measured (see Gredel 1994; 
Table 4. Estimated kinetic energy lost and variations in radiated energy for individual knots in HH 52 and HH 54.

\begin{tabular}{cccccc}
\hline \hline $\begin{array}{c}\text { knot } \\
\text { ID }\end{array}$ & $\begin{array}{c}\Delta K \\
\left(10^{38} \mathrm{erg}\right)\end{array}$ & $\begin{array}{c}\Delta E_{\mathrm{rad}}(\mathrm{HI}) \\
\left(10^{38} \mathrm{erg}\right)\end{array}$ & $\begin{array}{c}\Delta E_{\mathrm{rad}}(\mathrm{SII}) \\
\left(10^{38} \mathrm{erg}\right)\end{array}$ & $\begin{array}{c}\Delta E_{\mathrm{rad}}\left(\mathrm{H}_{2}\right) \\
\left(10^{38} \mathrm{erg}\right)\end{array}$ & $\begin{array}{c}\Delta E_{\mathrm{rad}}(\mathrm{tot}) \\
\left(10^{38} \mathrm{erg}\right)\end{array}$ \\
\hline HH 52 B1 & 70 & 1.1 & 0.08 & $0.2-0.4$ & $1.3-1.5$ \\
HH 52 C1 & 17 & 0.8 & 0.18 & 0.4 & 1.3 \\
HH 52 C2 & 3.5 & 0.35 & 0.1 & - & 0.4 \\
HH 54 H1 & 8.8 & 2.9 & 0.2 & - & 3.0 \\
HH 54 H2 & 9.6 & 2.8 & 0.12 & 0.9 & 3.8 \\
HH 54 H3 & 57 & 2.7 & 0.2 & 2 & 4.8 \\
HH 54 G & $-18^{a}$ & 4.0 & 0.02 & - & 4.0 \\
HH 54 G0 & 26 & 0.8 & $<0.01$ & - & 0.8 \\
\hline
\end{tabular}

Notes: ${ }^{a}$ here the kinetic energy is increased from the interaction with G0.

Giannini et al. 2006; Caratti o Garatti 2006). This corresponds to a luminosity of $L_{\mathrm{FeII}} \sim 4 \times 10^{29} \mathrm{erg} \mathrm{s}^{-1}$ (adopting the same physical parameters used for the other ions). This means that even assuming a luminosity variability equal to the knot luminosity the radiated energy in FeII would be $10^{36}-10^{37} \mathrm{erg}$, which is one or two orders of magnitude less than the HI contribution. The contribution from [S II] is usually smaller, down to two orders of magnitude (see Table 4). Therefore, among the considered atomic lines, HI radiates most of the energy observed in such a variability. This is well visible from the flux variability in Figs. 8, 9 (see also figures in Appendix C), where $\mathrm{H} \alpha$ usually exhibits the largest flux variability. This is possibly due to the different cooling times of the two atomic species (a few years for $\mathrm{HI}$ and 30 years for [S II], see e.g. Hartigan et al. 2001, 2005). As a consequence, usually $\mathrm{H} \alpha$ traces morphological changes better and faster than [S II] does.

Conversely, the $\mathrm{H}_{2}$ emission, when observed, partially contributes to the gas cooling, but with longer timescales. We note, however, that only part of the $\mathrm{H}_{2}$ variability was estimated (i.e. form the warm component $\sim 2000 \mathrm{~K}$ traced by the $2.12 \mu \mathrm{m}$ line), and we are likely missing the contribution of the cold $\mathrm{H}_{2}$ component that can contribute up to $50 \%$ to the whole radiative cooling (Caratti o Garatti et al. 2008).

It is not possible to properly evaluate the remaining molecular contribution, as for the CO. However it should not be greater than the atomic one, since the observed medium is partially ionised. Therefore it is reasonable to conclude that a large part of the kinetic energy lost in between two working surfaces is converted in radiated energy. This also means that the energy is rapidly converted, the shock dissociates the $\mathrm{H}_{2}$, and we observe the HI emission. After a few years, the temperature drops, and the $\mathrm{H}_{2}$ emission is then detected.

However, in some cases, the kinetic energy is not completely radiated but can be partially transferred to the medium or to the knots ahead of the flow, as shown in the interaction between knots HH 54 G0 and G, for example. Here, the kinetic energy lost by the bullet (G0) is mostly ceded to the target (G) and only partially lost $(\sim 20 \%)$ in the radiative process. In fact, the energy balance of the system $\left(\Delta K(\mathrm{G} 0)-\Delta K(\mathrm{G})-\Delta E_{\mathrm{rad}}(\mathrm{G} 0+\mathrm{G})\right)$ is close to zero, as can be derived from Table 4.

Finally, there are some knots that show flux variability but no change in velocity (inside the error bars). In some cases, where the flux decreases, we are observing the radiative cooling of the knot, but the momentum of the knot is conserved. In other cases, where the flux increases (see e.g. HH 54 A1 and A8 in Appendix C), we have estimated the amount of kinetic energy needed to produce the observed $\Delta E_{\mathrm{rad}}$. As a result the variation in velocity that produces such a $\Delta K$ is well below the error bars that we measure in our PMs.

\subsubsection{Cooling time and velocity variability}

Our set of observations indicate apparent acceleration and deceleration for several knots, but just a few of them seem to be genuine. Often the observed velocity changes do not reflect true fluid velocity variations, but merely phase velocity effects (see e.g. Bally et al. 2002), wherein some portions of the postshock gas fade and others brighten. This is particularly true when the elapsed time between two examined images is longer than the post-shock cooling time of the analysed knot.

We can thus compare the timescale of the observed variability with the expected atomic and molecular cooling times. The cooling time is

$t_{\mathrm{c}}=\frac{T}{|\mathrm{~d} T / \mathrm{d} t|} \sim \frac{n k_{\mathrm{B}} T}{\Lambda}$

where $T$ is the temperature, $k_{\mathrm{B}}$ the Boltzmann constant, and $\Lambda$ the cooling rate (in erg $\left.\mathrm{cm}^{-3} \mathrm{~s}^{-1}\right)^{11}$. To estimate $t_{\mathrm{c}}$, we use the $\mathrm{H}_{2}$ cooling rates from Shull \& Hollenbach (1978), and the atomic cooling coefficients from Cox \& Tucker (1969) and Gnat \& Sternberg (2007). For the $\mathrm{H}_{2}$ we consider a range of densities (from $10^{3}-10^{5} \mathrm{~cm}^{-3}$ ) and temperatures (from 2000 to $3000 \mathrm{~K}$ ). As a result, the $\mathrm{H}_{2}$ cooling time ranges from $\sim 1$ to $9 \mathrm{yr}$, where the shorter value is obtained for higher temperatures and densities. For the atomic component, we consider an average $N_{\mathrm{H}}$ of $10^{3} \mathrm{~cm}^{-3}$ and $T_{\mathrm{e}}$ ranging from 10000 to $25000 \mathrm{~K}$. We thus obtain $1 \leq t_{\mathrm{c}}(\mathrm{H} \alpha) \geq 6 \mathrm{yr}$. The cooling time of the [S II] is almost one order of magnitude longer than $t_{\mathrm{c}}(\mathrm{H} \alpha)$.

The complex proper motion variations may then indicate the combined effects of photometric and morphological changes, occurring on timescales comparable to the cooling time in the postshock gas, combined with the detection of collisions of fast, fragmented ejecta overrunning slower moving fragmented ejecta. The highly fragmented morphology of these shocks and the very short acceleration/deceleration timescales $(\sim 10 \mathrm{yrs})$ indicate that various types of instabilities (cooling instabilities, Vinsniac instabilities, etc.) may have caused the flow elements to fragment into tiny, dense clumps surrounded by lower density plasma or gas. As a fast shrapnel over-takes slower shrapnel, both accelerating and decelerating post-shock fluid elements are observed. Such timescales could be actually good measurements of the size-scales of the clumps in the colliding fluids.

\subsubsection{Direction variability and flow deflection}

Our PM analysis clearly indicates that part of the HH 52 flow is deflected. Some knots in the HH $54 \mathrm{G}$ group have been observed

\footnotetext{
11 Alternatively the cooling coefficient $\Lambda_{\mathrm{c}}$ (in $\mathrm{erg} \mathrm{cm}^{3} \mathrm{~s}^{-1}$ ) can be used and Eq. (3) becomes: $t_{\mathrm{c}} \sim \frac{k_{\mathrm{B}} T}{n \Lambda_{\mathrm{c}}}$.
} 


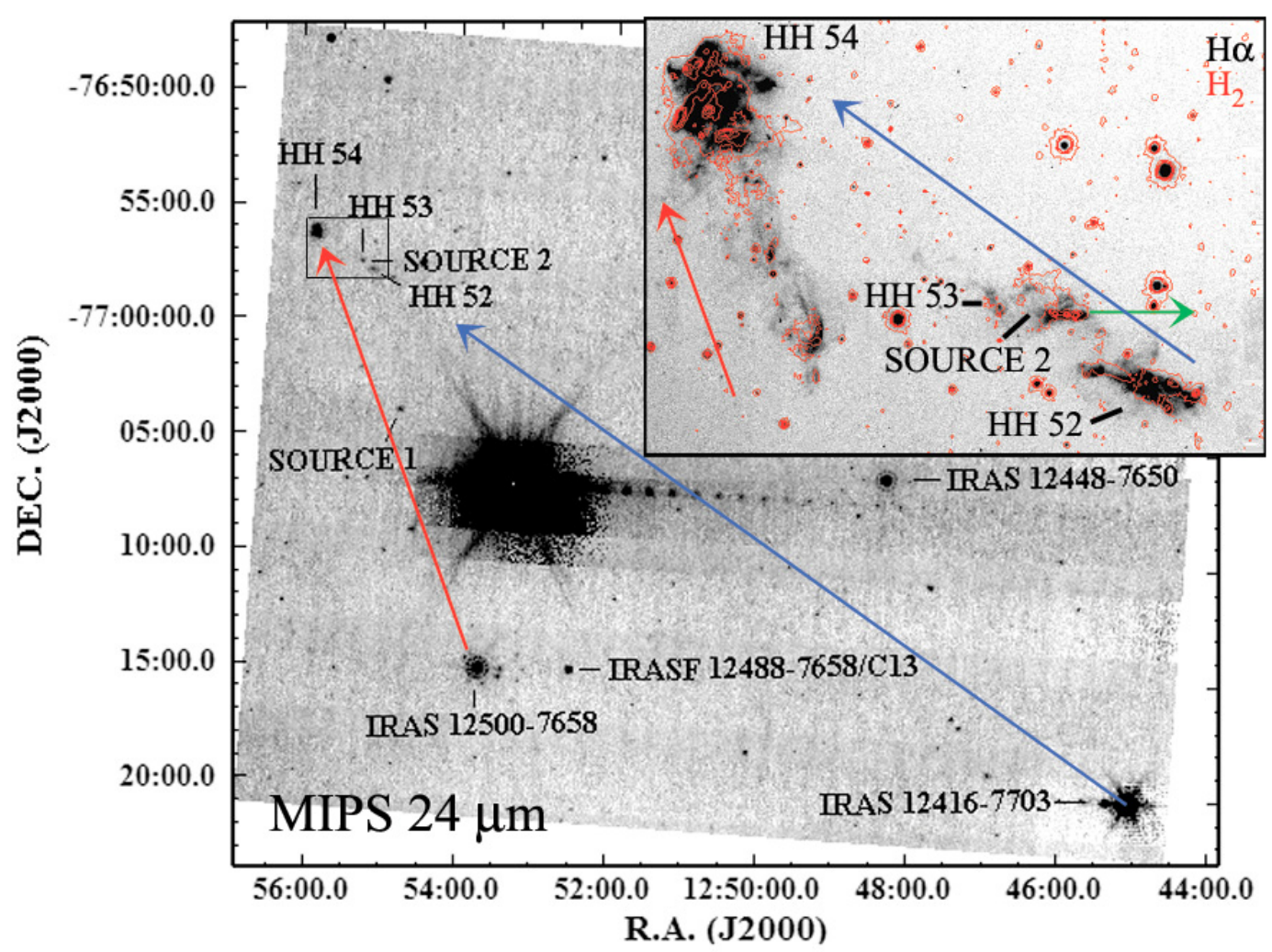

Fig. 15. Summary figure. Spitzer-MIPS $24 \mu \mathrm{m}$ map of the investigated Cha II region, indicating the candidate exciting sources, the outflows, and their orientations. A rectangular box indicates the position of the upper-right inset, where the $\mathrm{H} \alpha$ EMMI 2006 image along with the $\mathrm{H}_{2}$ SofI 199 image contours of the three outflows are reported.

to change their direction "in real time", due to the interaction with other colliding knots moving faster along the flow. In this case, most of the kinetic energy of the impacting knot (bullet) is transferred to the knot ahead (target), as shown in the previous section. To cause the deflection, the bullet trajectory is slightly off-axis with respect to the target. All this implies a time variability in the ejection velocity and a small precession of the flow, as well (see e.g. Raga et al. 1993; Völker et al. 1999).

Other knots, such as HH54 A, C, M, or HH52 G, F, exhibiting different PAs of $20^{\circ}-40^{\circ}$ with respect to the main flow, were probably deflected earlier, and it is not evident how this bending originated. However, it is not clear whether this "bullettarget" mechanism would remarkably deflect massive features like HH 54 A. Such a deflection is more likely the result of a collision of the flow with a dense cloud or dense clumps in the medium, as also observed in HH 110 (Reipurth et al. 1996) and in several numerical simulations (see e.g. de Gouveia Dal Pino 1999; Raga et al. 2002; Baek et al. 2008). In this scenario the deflected beam initially describes a $\mathrm{C}$-shaped trajectory around the curved jet/cloud contact discontinuity; later, when the jet has penetrated most of the cloud/clump extension, the flow resumes its original direction of propagation. This could be the reason we observe both a deflected (HH $54 \mathrm{~A}$ ) beam and a continuation of the flow (HH $54 \mathrm{G}$ and $\mathrm{C}$ ).

The observed velocities in the deflected beam are between $30 \%$ and $60 \%$ lower than those detected in HH 52. This is consistent with the values predicted by the simulations (see e.g. de Gouveia Dal Pino 1999; Baek et al. 2008) with $v_{\text {fin }}=v_{\text {in }} \cos \theta$, where $\theta$ is the deflection angle, $v_{\text {fin }}$ and $v_{\text {in }}$ are the deflected and incident velocities, respectively. Also the predicted ratio between jet and ambient medium $\left(n_{\mathrm{j}} / n_{\mathrm{m}} \leq 10^{-2}\right)$ agrees with our observations (see Sects. 4.7 and 4.8). Therefore the chaotic
Table 5. Lengths and dynamical age of the flows.

\begin{tabular}{cccc}
\hline \hline $\begin{array}{c}\text { Knot } \\
\text { ID }\end{array}$ & $\begin{array}{c}\text { Possible } \\
\text { exciting source }\end{array}$ & $\begin{array}{c}\text { Length } \\
(\mathrm{pc})\end{array}$ & $\begin{array}{c}\tau_{\text {dyn }} \\
\left(10^{3} \text { yrs }\right)\end{array}$ \\
\hline HH 52 A & IRAS 12416-7703 & 3.9 & 39 \\
HH 54 C & IRAS 12416-7703 & 4.2 & 63 \\
HH 54 G & IRAS 12416-7703 & 4.2 & 41 \\
HH 54 A & IRAS 12416-7703 & 4.2 & 63 \\
HH 53 A-C & Source 2 & $0.1-0.2$ & $0.3-0.8$ \\
HH 54 Y & IRAS 12500-7658 & 2.1 & 18 \\
HH 54 K-E & IRAS 12500-7658 & 2.2 & 43 \\
\hline
\end{tabular}

structure and PMs measured in HH 54 should be interpreted not only as the simple superposition of two distinct outflows but also as the result of a deflection in the HH 52 flow.

\subsection{Length, dynamical age of the outflows, counterflows}

Assuming that IRAS 12416-7703, Source 2, and IRAS 125007658 are the driving sources of HH 52, HH 53, and HH 54, respectively, we can infer two important parameters of the flows from our kinematical analysis: the spatial length and the dynamical age $\left(\tau_{\text {dyn }}\right)$, which is the ratio between the distance of the $\operatorname{knot}(\mathrm{s})$ from the YSO (corrected for the inclination angle $i$ ) and the total velocity. The inferred results are reported in Table 5. Additionally, Fig. 15 summarises the results, showing the candidate exciting sources, the outflows, and their orientations. Two out of the three outflows, namely HH 52 and HH 54, would be parsec scale outflows, with a length of about $4 \mathrm{pc}$ and $2.2 \mathrm{pc}$, respectively. The dynamical age of the flows would then be up to $6 \times 10^{4} \mathrm{yr}$ (HH 52 flow, knots HH54 A and C), and up to 
$4 \times 10^{4} \mathrm{yr}(\mathrm{HH} 54$ flow, knots E, K). On the other hand, if we assume Source 2 as the HH 53 driving source (but this is highly questionable), here the flow has a smaller extension $0.1-0.2 \mathrm{pc}$ and a shorter dynamical age $\left(<10^{3} \mathrm{yr}\right)$.

The detection of parsec scale outflows from Class 0 and I (e.g. Reipurth et al. 1997; Eislöffel 2000) as from Class II YSOs (McGroarty \& Ray 2004) is quite common. It is also not surprising that these flows have $4-6 \times 10^{4}$ years of age. What is indeed peculiar is that no further emission is detected between the parsec scale flows and the possible exciting sources. This could mean that the ejection (and therefore also the accretion) activity in two of the YSOs suddenly stopped or at least was reduced several thousand years ago. A different explanation could be that part of the flow is not emitting (i.e. it is neutral), and it only becomes visible when it shocks the slow material moving ahead. This seems to be the case of the HH $52 \mathrm{~B}$ and $\mathrm{C}$ regions, to the rear of the HH 52 flow, where we observe the formation of new knots.

Another puzzling question is the absence of a counterjet in all three flows. The red-shifted $\mathrm{CO}$ emission between $\mathrm{HH} 52$ and HH 54 (see Knee 1992) could be the HH 53 missing counterflow. However, we do not detect any jet in our images. Also for the two parsec scale flows, no emission is observed along the possible counterflow axes in Spitzer, 2MASS, or DSS images. Alcalá et al. (2008) report the discovery of a faint $\mathrm{H} \alpha$ emission, located $\sim 20^{\prime}$ to the SW of IRAS 12496-7650, but it is not aligned to the HH 54 flow. Finally, the Spitzer maps do not cover the hypothetical coordinates of HH 52 and HH 54 counterflows. According to CO maps of the Cha II cloud (see e.g. Hayakawa et al. 2001), both these coordinates are close to the southern cloud edge. Thus, because of the very low medium density, it is possible that the ejected matter cannot produce any shock.

\section{Conclusions}

We have presented a detailed multi-epoch (20 years baseline) kinematical investigation of $\mathrm{HH} 52,53$, and 54 at optical and near-IR wavelengths, along with medium (optical) and highresolution (NIR) spectroscopical analysis, probing the kinematical and physical conditions of the gas along the flows. We investigated flux and velocity variability with time of the flows, reporting the first detections of acceleration and deceleration of protostellar jets. We find that there is a correlation, at least in some cases, between the flux and the velocity variabilities observed along the flows. Finally, we discussed the possible flow exciting sources. The main results of this work are the following:

- The PM analysis reveals that there are three distinct outflows, partially overlapping. The first outflow (HH 54 flow) is oriented NNE with a position angle of $\sim 22^{\circ}$, delineated by the HH 54 streamer, and includes some of the knots in the main body of HH 54. The second (HH 52 flow) follows an NE direction with a position angle of $\sim 55^{\circ}$, grouping $\mathrm{HH} 52$ and part of HH 53 and 54 knots. The third (HH 53 flow), delineated by the three brightest knots of HH 53, is moving in an E-W direction. The HH 52 and 53 outflows partially overlap and seem to converge on the HH 54 bow shock.

- With a few exceptions, the derived PMs have similar values in the the optical filters $(\mathrm{H} \alpha,[\mathrm{S} \mathrm{II}])$ ranging between $0.007^{\prime \prime} \mathrm{yr}^{-1}$ and $\sim 0.097^{\prime \prime} \mathrm{yr}^{-1}$, corresponding to a tangential velocity between 6 and $82 \mathrm{~km} \mathrm{~s}^{-1}$ at a distance of $180 \mathrm{pc}$. On average the $\mathrm{H}_{2}$ PMs have lower values (from 0.007 to $0.041^{\prime \prime} \mathrm{yr}^{-1}$ ).
- Measured radial velocities of the atomic components (both optical and NIR) range between -20 and $-120 \mathrm{~km} \mathrm{~s}^{-1}$ with variations similar to the PMs. Spatial velocities of the knots vary from $50 \mathrm{~km} \mathrm{~s}^{-1}$ to $120 \mathrm{~km} \mathrm{~s}^{-1}$. The inclinations of the three flows are $58 \pm 3^{\circ}, 84 \pm 2^{\circ}$, and $67 \pm 3^{\circ}$ (HH 52, HH 53, and $\mathrm{HH} 54$, respectively).

- In 20 years, about $60 \%$ of the observed knots in the flows show flux variability. Moreover, our set of observations indicates acceleration and deceleration in several measurements, but just some of them seem to be reliable. These knots are working surfaces or interacting knots. In this case a relevant flux variability is observed as well. We argue that both flux and velocity variability are related and that part of or all the kinetic energy lost by the decelerating knots is successively radiated. However, the complex proper motion variations may also indicate the combined effects of photometric and morphological changes, occurring on timescales comparable to the cooling time in the post-shock gas, combined with the detection of collisions of fast, fragmented ejecta overrunning slower moving fragmented ejecta.

- The physical parameters from the diagnostic are quite homogeneous along and among the three flows. The analysis indicates the presence of very light $\left(N_{\mathrm{H}} \sim 10^{3} \mathrm{~cm}^{-3}\right)$, ionised $\left(X_{\mathrm{e}} \sim 0.2-0.6\right)$, and hot $\left(T_{\mathrm{e}} \sim 14000-26000 \mathrm{~K}\right)$ jets, impacting against a denser medium.

- The measured mass flux rates of the knots span from $10^{-9} M_{\odot} \mathrm{yr}^{-1}$ to some $10^{-7} M_{\odot} \mathrm{yr}^{-1}$, where the highest values are detected in the HH 52 flow.

- Several knots are deflected, at least in the HH52 flow. At least for a couple of them (HH 54 G and G0), the deflection originates in the collision of the two (what we call a bullettarget deflection mechanism). However, for the more massive parts of the flow, the deflection is more likely the result of the flow collision with a denser cloud or clump.

- We investigated the possible driving sources of the flows, comparing knot PMs and mass flux rates with the position, Class and bolometric luminosity of the source candidates. We indicated three possible candidates, IRAS 124167703, Source 2, and IRAS 12500-7658 for HH 52, HH 53, and HH 54, respectively. Only IRAS 12500-7658 is, however, a reliable candidate.

Acknowledgements. We thank Reinhard Mundt for putting the first optical imaging and spectroscopy at our disposal. We are grateful to Juan Alcalá and Loredana Spezzi for fruitful discussions and for providing us with photometric data of the YSOs candidates. Finally we would like to thank the referee, John Bally, for his helpful comments, which really improved the manuscript. The present work was supported in part by the European Community's Marie Curie Actions-Human Resource and Mobility within the JETSET (Jet Simulations, Experiments and Theory) network under contract MRTN-CT-2004 005592. This publication has made use of data from the "From Molecular Cores to Planet-forming Disks" (c2d) Legacy project. This research has also made use of NASA's Astrophysics Data System Bibliographic Services and the SIMBAD database, operated at the CDS, Strasbourg, France, and the 2MASS data, obtained as part of the Two Micron All Sky Survey, a joint project of the University of Massachusetts and the Infrared Processing and Analysis Center/California Institute of Technology, funded by the National Aeronautics and Space Administration and the National Science Foundation.

\section{References}

Alcalá, J. M., Spezzi, L., Chapman, N., et al. 2008, ApJ, 676, 427 Allen, L. E., Calvet, N., D’Alessio, P., et al. 2004, ApJS, 154, 363 Bacciotti, F., \& Eislöffel, J. 1999, A\&A, 342, 717

Baek, C. H., Kim, J., \& Choi, M. 2009, ApJ, 690, 944

Bally, J., Heathcote, S., Reipurth, B., et al. 2002, ApJ, 123, 2627

Buzzoni, B., Delabre, B., Dekker, H., et al. 1984, ESO Messenger, 38, 9 
Cabrit, S., Edwards, S., Strom, S. E. \& Strom, K. M. 1990, ApJ, 354, 687 Cardelli, J. A., Clayton, G. C., \& Mathis, J. S. 1989, ApJ, 345, 245

Caratti o Garatti, A. 2006, Ph.D. Thesis, Universitá degli studi di Roma Tor Vergata

Caratti o Garatti, A., Giannini, T., Lorenzetti, D., et al. 2004, A\&A, 422, 141

Caratti o Garatti, A., Giannini, T., Nisini, B., \& Lorenzetti, D. 2006, A\&A, 449, 1077

Caratti o Garatti, A., Froebrich, D., Eislöffel, J., Giannini, T., \& Nisini, B. 2008, A\&A, 485, 137

Chen, H., Grenfell, T. G., Myers, P. C., \& Hughes, J. D. 1997, ApJ, 478, 295

Cox, D. P., \& Tucker, W. H. 1969, ApJ, 157, 1157

Davis, C. J., Eislöffel, J., \& Ray, T. P. 1994, ApJ, 426, 93

Davis, C. J., Smith, M. D., \& Eislöffel, J. 2000, MNRAS, 318, 747

Davis, C. J., Hodapp, K. W., \& Desroches, L. 2001, A\&A, 377, 285

De Colle, F., \& Caratti o Garatti, A. 2009, A\&A, in preparation

Dekker, H., Delabre, B., \& D'Orico, S. 1986, SPIE, 627, 393

van Dokkum, P. G. 2001, PASP, 113, 1420

Eislöffel, J. 2000, A\&A, 354, 236

Eislöffel, J., \& Mundt, R. 1994, A\&A, 284, 530

Eislöffel, J., Davis, C., Ray, T. P. \& Mundt, R. 1994, ApJ, 422, 91

Esteban, C., Peimbert, M., García-Rojas, J., et al. 2004, MNRAS, 355, 229

Evans, N. J., Allen, L. E., Blake, G. A., et al. 2003, PASP, 115, 965

Giannini T., McCoey, C., Nisini, B., et al. 2006, A\&A, 459, 821

Gnat, O., \& Sternberg, A. 2007, ApJS, 168, 213

de Gouveia Dal Pino, E. M. 1999, ApJ, 526, 862

Graham, J. A., \& Elias, J. H. 1983, ApJ, 272, 615

Graham, J. A., \& Hartigan, P. 1988, ApJ, 95, 4

Gredel, R. 1994, A\&A, 292, 580

Hamuy, M., Suntzeff, N. B., Heathcote, S. R., et al. 1994, PASP, 106, 566

Hartigan, P., Raymond, J., \& Hartmann, L. 1987, ApJ, 316, 323

Hartigan, P., Morse, J. A., \& Raymond, J. 1994, ApJ, 436, 125

Hartigan, P., Morse, J., \& Bally, J. 2000, AJ, 120, 1436

Hartigan, P., Morse, J., Reipurth, B., et al. 2001, ApJ, 559, L157

Hartigan, P., Heathcote, S., Morse, J., Reipurth, B., \& Bally, J. 2005, AJ, 130, 2197

Hayakawa, T., Cambrésy, L., Onishi, T., et al. 2001, PASJ, 53, 1109

Heathcote, S., Morse, J. A., Hartigan, P., et al. 1996, AJ, 112, 1141

Johansson, S. 1978, Phys. Scr., 18, 217

Knee, L. B. G. 1992, A\&A, 259, 283
Lavalley-Fouquet, C., Cabrit, S., \& Dougados, C. 2000, A\&A, 356, L41

Lidman, C., Gredel, R., \& Moneti, A. 1997, IRAC2b User Manual V1.5,

European Southern Observatory technical document7

Magazzu, A., Rebolo, R., \& Pavlenko, I. V. 1992, ApJ, 392, 159

McGroarty, F., \& Ray, T. P. 2004, A\&A, 420, 975

Moorwood, A., Cuby, J. G., \& Lidman, C. 1998a, The Messenger, 91, 9

Moorwood, A., Cuby, J. G., Biereichel, P., et al. 1998b, The Messenger, 94, 7

Mundt, R. 1985, in Protostars and planets II, ed. D. C. Black, \& M. S. Matthews (Tucson: Univ. Arizona Press), 414

Nisini, B., Bacciotti, F., Giannini, T., et al. 2005, A\&A, 441, 159

Osterbrock, D. E., Fulbright, J. P., Martel, A. R., et al. 1996, PASP, 108, 2770

Podio, L., Bacciotti, F., Nisini, B., et al. 2006, A\&A, 456, 189

Porras, A., Jorgensen, J. K., Allen, L. E., et al. 2007, ApJ, 656, 493

Raga, A., Cantó, J., \& Biro, S. 1993, MNRAS, 260, 163

Raga, A., de Gouveia Dal Pino, E. M., Noriega-Crespo, A., Minnini, P. D., \& Velázquez, P. F. 2002, A\&A, 392, 267

Reipurth, B., \& Bally, J. 2001, ARA\&A, 39, 403

Reipurth, B., Bally, J., Graham, J. A., Lane, A. P., \& Zealey, W. J. 1986, A\&A, 164,51

Reipurth, B., Raga, A. C., \& Heathcote, S. 1996, A\&A, 311, 989

Reipurth, B., Bally, J., \& Devine, D. 1997, AJ, 114, 2708

Rousselot, P., Lidman, C., Cuby, J. G., et al. 2000, A\&A, 354, 1134

Sandell, G., Zealey, W. J., Williams, P. M., et al. 1987, A\&A, 182, 237

Schwartz, R. D. 1977, ApJS, 35, 161

Schwartz, R. D. 1978, ApJ, 223, 884

Schwartz, R. D., \& Dopita, M. A. 1980, ApJ, 236, 543

Schwartz, R. D., Jones, B., \& Sirk, M. 1984, AJ, 89, 1735

Schwartz, R. D., Choen, M., \& Williams, P. M. 1987, ApJ, 322, 403

Shu, F. H., Najita, J. R., Shang, H., \& Li, Z.-Y. 2000, in Protostars and planets IV, ed. V. Mannings, A. P. Boss, \& S. S. Russel (Tucson: University of Arizona Press), 789

Shull, J. M., \& Hollenbach, D. J. 1978, ApJ, 220, 525

Spezzi, L., Alcalá, J. M., Frasca, A., et al. 2007, A\&A, 470, 281

Spezzi, L., Alcalá, J. M., Covino, E., et al. 2008, ApJ, 680, 1295

Storey, P. J., \& Hummer, D. G. 1995, MNRAS, 272, 41

Völker, R., Smith, M. D., Suttner, G., \& Yorke, H. W. 1999, A\&A, 343, 953

Vuong, M. H., Cambrésy, L., \& Epchtein, N. 2001, A\&A, 379, 208

Whittet, D. C. B., Prusti, T., Franco, G. A. P., et al. 1997, A\&A, 327, 1194

Young, E. K., Harvey, P. M., Brooke, T. Y., et al. 2005, ApJ, 628, 297 


\section{Appendix A: Morphology}

\section{A.1. $\mathrm{HH} 52-53$}

Our high resolution images allow us to disentangle the structure of the studied HH objects. Figures 4 (right panel) and A.1 show the HH 52 and 53 region in the three different filters. In $\mathrm{H} \alpha$ and [S II] HH 52 exhibits a roughly bow shape, where knots labelled A can be identified as the head of the shock (nucleus). The brightest knot (A1) is surrounded by several smaller structures, with knots $\mathrm{A} 3$ and $\mathrm{A} 4$ placed behind, while $\mathrm{A} 5$ and $\mathrm{A} 6$ are in front, elongated towards a group of separated knots (D1-D3) roughly E-W aligned. A faint emission (knot D4) is placed just in front of the nucleus. Groups of knots B and C constitute the right and left wings of the bow, respectively. The right wing appears particularly fragmented with knots B1-B3 representing a first condensation close to the nucleus. B4-B9 are located behind.

The $\mathrm{H}_{2}$ emission (Fig. A.1, bottom) shows a more amorphous shape. A semi-ring shaped region, roughly coincident with knots A2-A4, is located just behind the nucleus. Three more bright spots identify the two wings. The first emission coincides with $\mathrm{C} 1$ on the left wing, the second, on the right wing, extends to an area corresponding to B2 and B3 in the optical, and the third to B5 and B6 on the right wing as well. Finally, fainter and diffuse emission is detected on the D1-D3 knots.

In addition to the three HH 53 knots already known (A-C), we detect several more emissions coming from this region. Three more knots, mainly emitting in the $\mathrm{H} \alpha$ line (Fig. 4), are found along the main flow, forming an elongated S-shape chain of $\mathrm{E}-\mathrm{W}$ knots. We label $\mathrm{C} 1$ the emission between $\mathrm{C}$ and $\mathrm{A}$, and $\mathrm{B} 1$ and $\mathrm{B} 2$ those close to $\mathrm{B}$. These objects are not detected in our $\mathrm{H}_{2}$ images and two of them (knots $\mathrm{C} 1$ and $\mathrm{B} 1$ ) are just barely visible on the [S II] images. More diffuse emission, detected in all three filters, appears superimposed on the HH 53 main flow, connected to the HH 52 streamer. HH 53 I lies SW, towards HH 52, while $\mathrm{H}$ and $\mathrm{E}$ are situated $\mathrm{NE}$ in the opposite direction. Two brighter structures are located farther NE along the streamer. Group F, with a jet-like appearance in the optical filters, is approximately elongated towards $\mathrm{NE}$, also visible in $\mathrm{H}_{2}$. Knot $\mathrm{G}$, only visible in the optical, is approximatively located at the origin of the continuous emission of the streamer.

\section{A.2. $\mathrm{HH} 54$}

In Figs. 4 (left panel) and A.2 we show the HH 54 images and contours in $\mathrm{H} \alpha$, [S II] (left panel of Fig. A.2) and $\mathrm{H}_{2}$ (right panel of Fig. A.2). The morphology of the object appears extremely complex. Our images reveal several substructures. For the sake of simplicity, we divide the description of the region in two parts, the streamer, composed of groups X, Y, and Z, and the main body of HH 54, made up of the remaining knots.

HH 54 streamer shows an uncommon shape of a double helix, clearly visible in $\mathrm{H} \alpha$. The brightest helix is also well delineated in [S II], appearing as a wiggling jet fragmented in several knots. The morphology of the streamer closely resembles that of the HH 46/47 outflow (see e.g. Eislöffel \& Mundt 1994b; Heathcote et al. 1996), except for the presence of a second faint helix. The base of the streamer is X0, with knots X2 and X3 delineating the first bend of the bright helix. The path follows with $\mathrm{X} 4$. Y1 and Y2 delineate the second bend of the jet, that continues with Y4. The faint helix is delineated by X1, Y3, Y8, Y7 and Y6. Both the bright and faint structures end in two shocks ( $\mathrm{Z}$ and $\mathrm{Z1}$, respectively), that barely appear as a bow shock feature in $\mathrm{H}_{2}$ (Fig. A.2, right panel). Molecular hydrogen emission is also detected in knots X0, X2, Y1, and Y2. A further knot (T) complementary to the ionic emission is observed along the jet. Finally, some filaments (X4 A, Y5, R), detected at optical wavelengths, are located aside, detached from the main flow.

The morphology of the main body appears even more complicated than the streamer. In addition to the original knots (from A to K), observed in previous papers (Schwartz 1977; Schwartz \& Dopita 1980; Sandell et al. 1987), we detect more features (labelled L to S). Moreover, several knots present substructures, labelled here with numbers.

Group A at the bottom appears as bow-shaped in the optical, with a PA of about $75^{\circ}$. In $\mathrm{H}_{2}$ the group exhibits emission only in the northern part of the structure, only partially overlapping the atomic emission. A fainter arc-shaped emission (group Q), mainly visible in $\mathrm{H} \alpha$, is located east. Knots $\mathrm{P}$ (detected in the optical) and $\mathrm{S}$ (detected in $\mathrm{H}_{2}$ ) are located west, and together with knots $\mathrm{O}$ (farther north, visible in the optical) form the farthest point of the HH 52 streamer.

Towards north, more groups are detected. From east to west, almost coincident in all three wavelengths, we detect groups $\mathrm{K}$ and E, slightly elongated northward, then group B, and, finally group F, detected only in the optical. Farther NE an arc-like structure is identified by groups $\mathrm{J}$ and $\mathrm{M}$ in both optical and NIR.

In the north-eastern region, knot I and group $\mathrm{H}$ appear as optical jets with PAs of $\sim 45^{\circ}$ and $\sim 20^{\circ}$, respectively. An arc emission in $\mathrm{H}_{2}$ is delineated ahead of $\mathrm{I}$.

In the north-western region, we detect group $\mathrm{G}$, composed of five subsequent knots only detected in the optical (mainly in the $\mathrm{H} \alpha$ filter, see also Fig. 4 ), with a PA of $\sim 55^{\circ}$. At the end of this flow another group of knots $(\mathrm{C})$ is observed, with $\mathrm{C} 1$ and $\mathrm{C} 2$ already known, and the new knot $\mathrm{C} 3$ located on a straight line connecting knots $\mathrm{G}$ and $\mathrm{C} 2$.

It is worth to note that the identification in the NIR of knots C accomplished by Gredel (1994) (and then used in Caratti o Garatti et al. 2006; and in Giannini et al. 2006) is erroneous. Indeed the knots that were labelled in $\mathrm{H}_{2}$ as $\mathrm{C} 1$ and $\mathrm{C} 2$ have a similar appearance in the optical, but are situated about 6" SW. As a consequence, the knot previously known as $\mathrm{C} 1$ in the NIR coincides with knot $\mathrm{H} 2$ in the optical, while $\mathrm{C} 2$ roughly corresponds to $\mathrm{C} 1$ in the optical (see Figs. 4 and A.2). In this paper we use this nomenclature to avoid further confusion.

\section{Appendix B: Proper motions}

\section{B.1. $\mathrm{HH} 52-53$}

Knot position angles along HH 52 flow have a quite wide spread in their values $\left(30^{\circ}-80^{\circ}\right)$. The bulk of $\mathrm{HH} 52$ and other knots, as $\mathrm{HH} 53 \mathrm{C} 1, \mathrm{I}$, and $\mathrm{H}$, have a PA of $\sim 50^{\circ}$. On the other hand HH 52 A3, A4 and a few more knots as HH $53 \mathrm{~F}$ and G, proceed towards HH 54 with different PAs between $60^{\circ}$ and $80^{\circ}$. A completely different behaviour is found for the features located on HH 52 wings in the rear of the flow. Although following the bulk of the flow, they show a high degree of variability during the 20 years of observations, as reported in Appendix C. Such a variability, both due to local instabilities inside the flow and fast moving shocks overtaking HH 52 from behind, makes their identification in different epochs and a correct evaluation of the PMs and PAs sometimes difficult (see also Figs. 5 and 6).

In Fig. B. $1 \mathrm{H}_{2}$ proper motions are reported (see also Tables B.3 and B.4). Significant measurements in HH 52 were possible only for the three bright spots located in the wings. B2-B3 and C1 follow the flow as in the optical, with similar tangential velocities and position angles. 


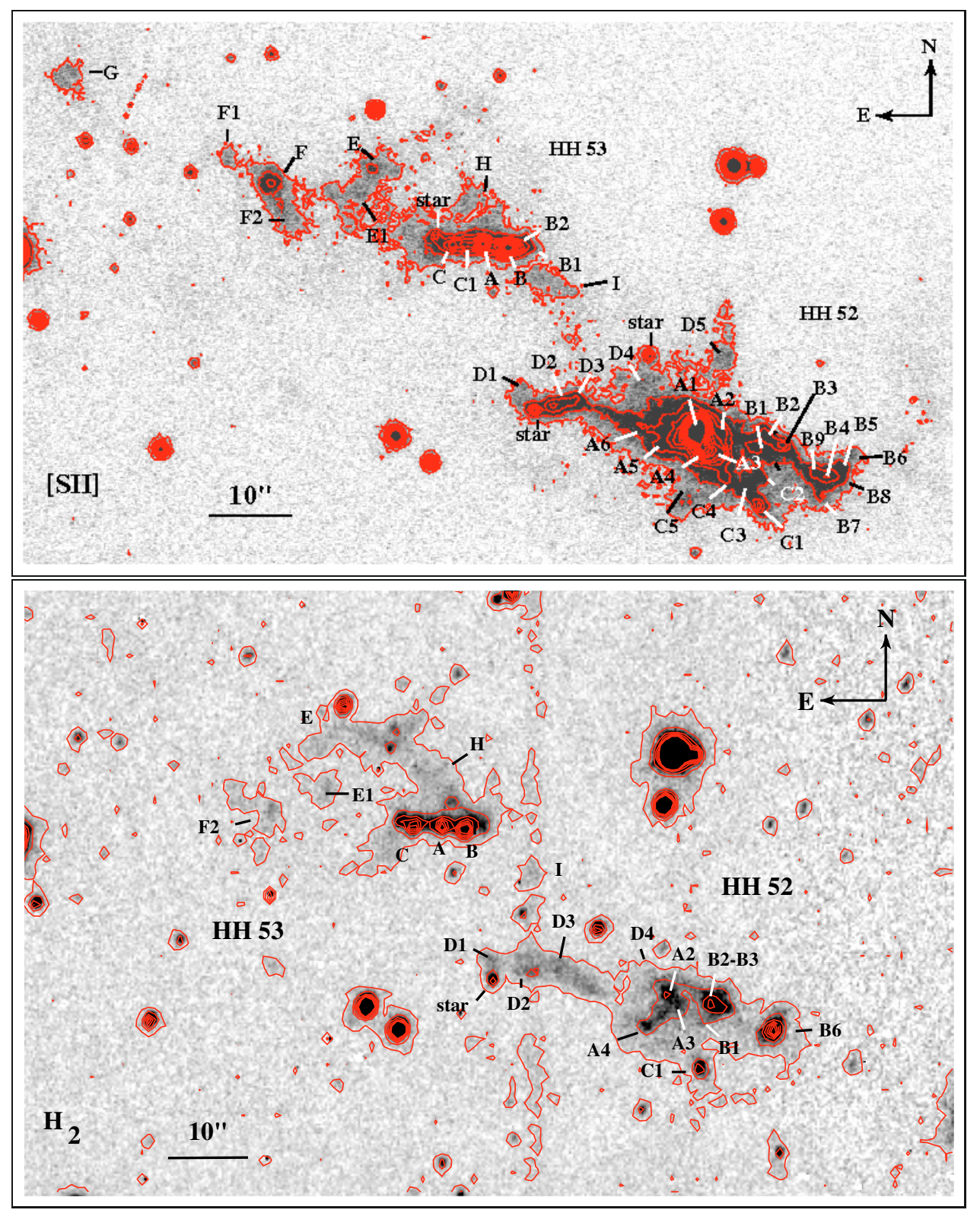

Fig. A.1. HH 52 and HH 53 regions with [S II] (EMMI 2006) (top), and $\mathrm{H}_{2}$ (SofI 1999) (bottom) filters. The labels indicate the position of the knots, including the newly detected ones. The contour levels of the $[\mathrm{S} \mathrm{III}]$ are $3,10,20,30,50,60,80,90,100,150,200 \times \sigma(\sim 4 \times$ $\left.10^{-17} \mathrm{erg} \mathrm{s}^{-1} \mathrm{~cm}^{-2} \operatorname{arcsec}{ }^{-2}\right) . \mathrm{H}_{2}$ levels are 3, 10, 20, 30, 40, 50, 60 $\times \sigma\left(\sim 10^{-16} \mathrm{erg} \mathrm{s}^{-1} \mathrm{~cm}^{-2} \operatorname{arcsec}^{-2}\right)$.

HH 53 A, B and C, in the HH 53 outflow, exhibit small PMs with PAs around $270^{\circ}$ also in the NIR. Here, however, the measurements are less significant due to the larger errors.

\section{B.2. $\mathrm{HH} 54$}

Proper motions in the HH 54 streamer range between $\sim 0.01$ and $0.07^{\prime \prime} \mathrm{yr}^{-1}$ (i.e. $v_{\tan } 8$ and $61 \mathrm{~km} \mathrm{~s}^{-1}$ ), where the highest velocities (between $\sim 30$ and $60 \mathrm{~km} \mathrm{~s}^{-1}$ ) are detected in the inner part of the flow and exhibit alternating PAs $\left(322^{\circ}-69^{\circ}\right)$, likely due to precession, with directions that oscillate around an average value of $\sim 20^{\circ}$.

Some features, located on the edge of the streamer, are not collimated and are drifting apart $\left(\mathrm{PA} \sim 90^{\circ}\right)$ at a lower velocity. At the tip of the streamer group $\mathrm{Z}$ appears almost stationary in $\mathrm{H} \alpha\left(v_{\tan } \sim 10 \mathrm{~km} \mathrm{~s}^{-1}\right)$, while in [S II] has a higher value of $\sim 30 \mathrm{~km} \mathrm{~s}^{-1}$.
Moving towards NE we observe a decrease in the tangential velocities from groups Q $\left(\sim 40 \mathrm{~km} \mathrm{~s}^{-1}\right)$ to $\mathrm{K}$ and $\mathrm{E}(\sim 20$ and $15 \mathrm{~km} \mathrm{~s}^{-1}$, respectively). From the PAs and inclinations (see Sect. 4.6) we can reasonably associate these knots with the HH 54 flow.

Group A appears as an expanding bow shock, towards ENE with an average direction of about $80^{\circ}$ and a mean $v_{\tan }$ of $\sim 30 \mathrm{~km} \mathrm{~s}^{-1}$. Knots A1, A2, A6 and A8 exhibit some variability, as reported in Appendix C. Because of the morphology, the direction, and the average inclination (see Sect. 4.6), the structure appears to be connected to knot P and to the HH 52 flow. Indeed part of the flow along the HH 52 streamer curves and shocks this region, producing group $\mathrm{O}$ as well (see also Fig. 1). $\mathrm{H}_{2}$ emission in HH 54 A (Fig. A.2) appears as an expanding arc, and its motion $\left(v_{\tan } \sim 30 \mathrm{~km} \mathrm{~s}^{-1}\right)$ has a different orientation depending on the position along the structure (Fig. B.1). Proceeding from east to west, we separately measured four regions, indicated in 

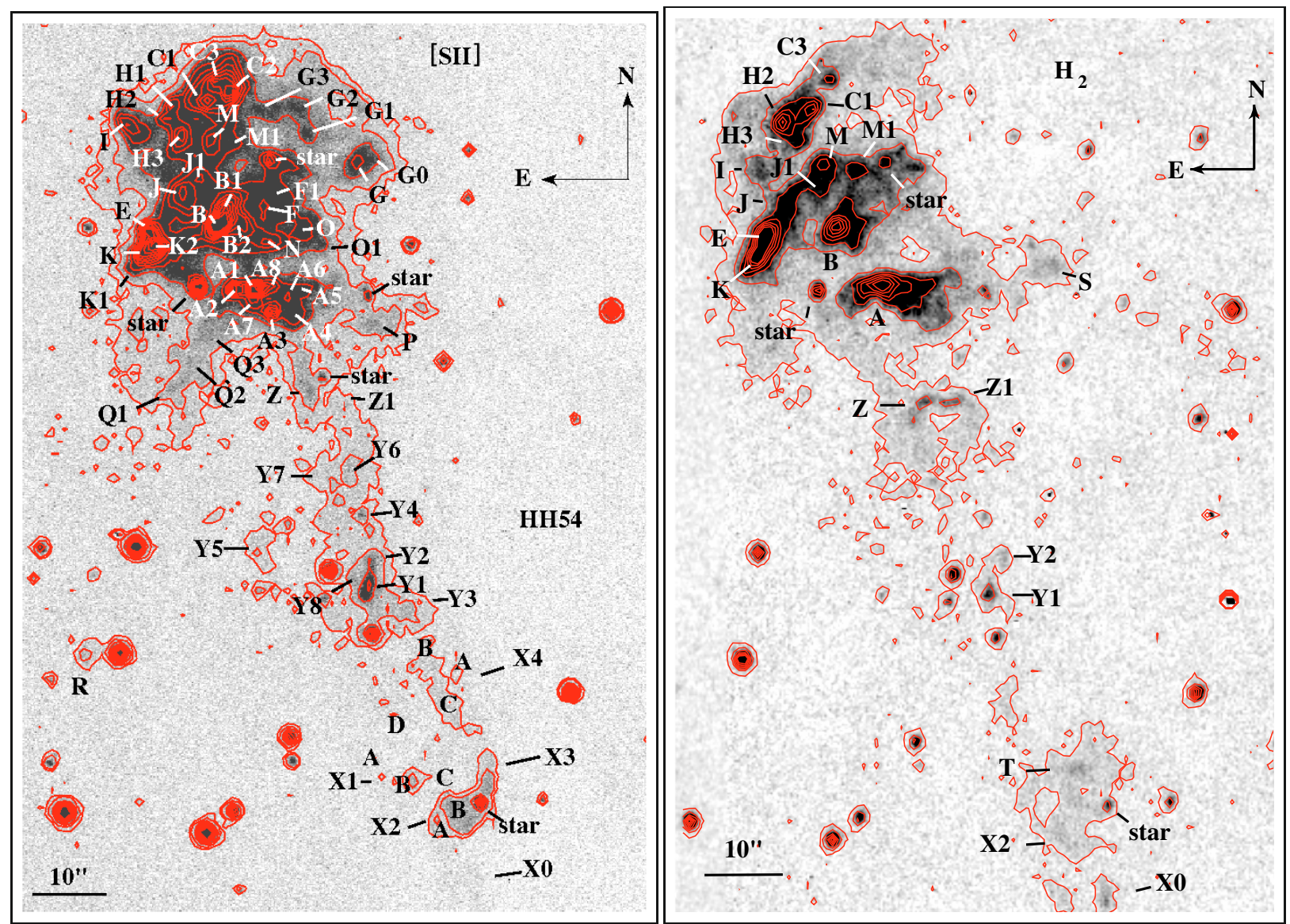

Fig. A.2. HH 54 regions with [S II] (EMMI 2006) (left) and $\mathrm{H}_{2}$ (SofI 1999) (right) filters. The labels indicate the position of the knots, including the newly detected ones. [S II] contour levels are 3, 10, 20, 30,40,50,60, 70, 80, 90, 100× the standard deviation to the mean background $\left(\sim 4 \times 10^{-17} \mathrm{erg} \mathrm{s}^{-1} \mathrm{~cm}^{-2}\right.$ arcsec $\left.{ }^{-2}\right) . \mathrm{H}_{2}$ contour levels are $3,10,20,30,40,50,60 \times$ the standard deviation to the mean background $\left(\sim 10^{-16} \mathrm{erg} \mathrm{s}^{-1} \mathrm{~cm}^{-2} \operatorname{arcsec}^{-2}\right)$.

Table B.4 as $\mathrm{A}_{1}, \mathrm{~A}_{2}, \mathrm{~A}_{3}$, and $\mathrm{A}_{4}$. The directions of the motions have different PAs with respect to the optical counterparts and it is not clear if the molecular emission comes from material swept up by the bow shock on one side (see e.g. HH 219 , Caratti o Garatti et al. 2004) or from the HH 54 streamer.

The faintest component of the HH 52 streamer follows a straight trajectory (of $\sim 55^{\circ}$ ) and impacts ahead, producing groups $\mathrm{HH} 54 \mathrm{G}$ and then $\mathrm{C}$. We observe a sudden deceleration of the flow along the HH 52 streamer (from $\sim 65$ to $30 \mathrm{~km} \mathrm{~s}^{-1}$ ) as it collides with the leading material, that is partially deflected sideways ( $\mathrm{PA} \sim 80^{\circ}$ ). G0 is part of such a fast flow, that shocks at the end of a slow moving component (knot G), changing its direction (see also Sect. 4.4). This dynamic is also visible in knots G1 and G3, that are not aligned with the flow, moving with a PA of $\sim 80^{\circ}$ and $\sim 65^{\circ}$, respectively. Group $\mathrm{C}$ ahead of $\mathrm{G}$ is proceeding in the same direction at similar tangential velocities $\left(25-30 \mathrm{~km} \mathrm{~s}^{-1}\right)$. Surprisingly, knot C3, at the tip of the flow, slightly moves in the opposite direction in both optical and NIR filters (PA $\sim 250^{\circ}$ and $v_{\tan } \sim 15 \mathrm{~km} \mathrm{~s}^{-1}$ ). This behaviour is observed also in knot $\mathrm{K} 1$, on the opposite side of the HH object, showing nearly a reverse motion with respect to the main flows.

Velocities of knots $\mathrm{M}$ are quite low (about $20 \mathrm{~km} \mathrm{~s}^{-1}$ ) with a position angle around $80^{\circ}$. The direction and the velocities suggest that they could be part of the same outflow, generated by the same deflection mechanism.

Groups $\mathrm{H}$, I and $\mathrm{J}$, on the northeast side, are moving NE $\left(\mathrm{PA} \sim 45^{\circ}\right.$ ) with a $v_{\tan }$ between 10 and $50 \mathrm{~km} \mathrm{~s}^{-1}$.
Group N, in the central region of the main body, could be part of the HH 54 flow, with a $v_{\tan } \sim 20-25 \mathrm{~km} \mathrm{~s}^{-1}$ and a PA $\sim 320^{\circ}$.

It is difficult to determine the membership of several knots (as B or F groups) in the central part of the HH object. The bulk of knot B possibly moves slightly towards ESE, while B1, few arcseconds north, seems to proceed in the opposite direction. Also $\mathrm{F}$ and $\mathrm{F} 1$ show a similar behaviour.

The molecular component of knot B presents distinct internal motions. We detect three components, reported in Table B.4 $\left(\mathrm{B}_{1}, \mathrm{~B}_{2}, \mathrm{~B}_{3}\right.$, from north to south). $\mathrm{B}_{1}$ appears as a bright arc in the high spatial resolution ISAAC images and it is moving towards NNE $\left(\sim 20^{\circ}\right) . \mathrm{B}_{2}$ and $\mathrm{B}_{3}$ are proceeding towards ESE $\left(130^{\circ}-140^{\circ}\right)$ as in the optical. In this case we are possibly observing the motion of the two different outflows.

\section{Appendix C: Flux and velocity variability}

\section{C.1. $\mathrm{HH} 52$}

\section{C.1.1. $\mathrm{HH} 52 \mathrm{~B}$ and $\mathrm{C}$}

Both wings of HH 52 bow-shock show a high degree of variability. As an example of such a variability, we show in Fig. C.1 a close up of HH52 in 1993 and $2006 \mathrm{H} \alpha$ calibrated images. Changes in morphology and flux are easy to recognise, especially for the knots of group B.

HH 52 B1 and B2 come from the fragmentation of a single structure around 1987. The first knot is moving ESE with 
A. Caratti o Garatti et al.: Time-variability in the Chamaeleontis II outflows, Online Material p 4

Table B.1. Proper Motions in HH 52 and $\mathrm{HH} 53$ - H $\alpha$ and [S II] - parameters derived from the linear fit.

\begin{tabular}{|c|c|c|c|c|c|c|c|c|c|c|}
\hline \multirow{2}{*}{$\begin{array}{l}\text { Knot ID } \\
\text { HH 52 A1 }\end{array}$} & \multicolumn{3}{|c|}{$\begin{array}{c}\alpha(2000.0) \\
(\mathrm{hms})\end{array}$} & \multicolumn{3}{|c|}{$\begin{array}{c}\delta(2000.0) \\
\left({ }^{\circ},{ }^{\prime} \prime \prime\right)\end{array}$} & \multirow{2}{*}{$\begin{array}{c}\mathrm{PM} \\
\left({ }^{\prime \prime} \mathrm{yr}^{-1}\right) \\
0.055 \pm 0.002\end{array}$} & \multirow{2}{*}{$\begin{array}{c}\text { PA } \\
\left({ }^{\circ}\right) \\
42 \pm 2\end{array}$} & \multirow{2}{*}{$\begin{array}{c}\begin{array}{c}v_{\tan } \\
\left(\mathrm{km} \mathrm{s}^{-1}\right)\end{array} \\
48 \pm 1\end{array}$} & \multirow{2}{*}{$\begin{array}{l}\text { Filter } \\
\text { [S II] }\end{array}$} \\
\hline & 12 & 55 & 07.6 & -76 & 57 & 52 & & & & \\
\hline & 12 & 55 & 07.6 & -76 & 57 & 52 & $0.052 \pm 0.002$ & $52 \pm 2$ & $44 \pm 2$ & $\mathrm{H} \alpha$ \\
\hline HH 52 A2 & 12 & 55 & 06.7 & -76 & 57 & 52 & - & - & - & {$[\mathrm{S}$ II $], \mathrm{H} \alpha$} \\
\hline \multirow[t]{2}{*}{ HH 52 A 3} & 12 & 55 & 06.7 & -76 & 57 & 55 & - & - & - & [S II] \\
\hline & 12 & 55 & 06.7 & -76 & 57 & 55 & $0.047 \pm 0.004$ & $70 \pm 4$ & $40 \pm 3$ & $\mathrm{H} \alpha$ \\
\hline \multirow[t]{2}{*}{ HH 52 A4 } & 12 & 55 & 07.4 & -76 & 57 & 54 & $0.059 \pm 0.009$ & $70 \pm 9$ & $50 \pm 8$ & [S II] \\
\hline & 12 & 55 & 07.5 & -76 & 57 & 55 & $0.048 \pm 0.002$ & $71 \pm 3$ & $40 \pm 2$ & $\mathrm{H} \alpha$ \\
\hline \multirow[t]{2}{*}{ НH 52 А 5} & 12 & 55 & 09.1 & -76 & 57 & 54 & $0.028 \pm 0.002^{a}$ & $62 \pm 3$ & $24 \pm 1^{a}$ & [S II] \\
\hline & 12 & 55 & 09.1 & -76 & 57 & 54 & $0.056 \pm 0.003^{a}$ & $67 \pm 3$ & $48 \pm 2^{a}$ & $\mathrm{H} \alpha$ \\
\hline \multirow[t]{2}{*}{ HH 52 A6 } & 12 & 55 & 09.5 & -76 & 57 & 52 & - & - & - & [S II] \\
\hline & 12 & 55 & 09.5 & -76 & 57 & 52 & $0.035 \pm 0.003$ & $78 \pm 4$ & $29 \pm 3$ & $\mathrm{H} \alpha$ \\
\hline HН 52 B1 & 12 & 55 & 05.6 & -76 & 57 & 54 & $0.046 \pm 0.002^{a}$ & $116 \pm 3$ & $39 \pm 2^{a}$ & [S II] \\
\hline & 12 & 55 & 05.2 & -76 & 57 & 55 & $0.031 \pm 0.004^{a}$ & $111 \pm 6$ & $26 \pm 3^{a}$ & $\mathrm{H} \alpha$ \\
\hline HH 52 B2 & 12 & 55 & 05.3 & -76 & 57 & 53 & $0.053 \pm 0.002^{a}$ & $2 \pm 3$ & $45 \pm 2^{a}$ & [S II] \\
\hline & 12 & 55 & 05.2 & -76 & 57 & 53 & $0.062 \pm 0.002^{a}$ & $1 \pm 3$ & $53 \pm 2^{a}$ & $\mathrm{H} \alpha$ \\
\hline HH 52 B3 & 12 & 55 & 04.6 & -76 & 57 & 54 & $0.038 \pm 0.002^{a}$ & $71 \pm 3$ & $32 \pm 2^{a}$ & [S II] \\
\hline & 12 & 55 & 04.6 & -76 & 57 & 54 & $0.044 \pm 0.003$ & $80 \pm 3$ & $37 \pm 2$ & $\mathrm{H} \alpha$ \\
\hline HH 52 B4 & 12 & 55 & 03.0 & -76 & 57 & 57 & $0.017 \pm 0.002^{a}$ & $63 \pm 7$ & $14 \pm 2^{a}$ & [S II $]$ \\
\hline & 12 & 55 & 03.0 & -76 & 57 & 58 & - & - & - & $\mathrm{H} \alpha$ \\
\hline HH 52 B5 & 12 & 55 & 02.9 & -76 & 57 & 58 & $0.011 \pm 0.002^{a}$ & $309 \pm 10$ & $10 \pm 2^{a}$ & [S II] \\
\hline & 12 & 55 & 02.7 & -76 & 57 & 58 & $0.097 \pm 0.003$ & $195 \pm 2$ & $82 \pm 2$ & $\mathrm{H} \alpha$ \\
\hline HH 52 B6 & 12 & 55 & 02.2 & -76 & 57 & 55 & $0.034 \pm 0.002$ & $257 \pm 3$ & $29 \pm 1$ & [S II] \\
\hline & 12 & 55 & 02.1 & -76 & 57 & 55 & $0.033 \pm 0.001$ & $249 \pm 2$ & $28 \pm 1$ & $\mathrm{H} \alpha$ \\
\hline HH 52 B7 & 12 & 55 & 03.4 & -76 & 58 & 00 & $0.033 \pm 0.003$ & $157 \pm 6$ & $28 \pm 3$ & [S II] \\
\hline & 12 & 55 & 03.2 & -76 & 58 & 00 & $0.062 \pm 0.003$ & $152 \pm 3$ & $52 \pm 2$ & $\mathrm{H} \alpha$ \\
\hline HH 52 B8 & 12 & 55 & 02.4 & -76 & 57 & 57 & $0.019 \pm 0.005^{a}$ & $288 \pm 11$ & $16 \pm 4^{a}$ & [S II] \\
\hline & 12 & 55 & 02.5 & -76 & 57 & 56 & - & - & - & $\mathrm{H} \alpha$ \\
\hline HН 52 B9 & 12 & 55 & 03.6 & -76 & 57 & 57 & $0.045 \pm 0.011$ & $82 \pm 15$ & $38 \pm 9$ & $\mathrm{H} \alpha$ \\
\hline HH 52 C1 & 12 & 55 & 05.4 & -76 & 58 & 01 & $0.037 \pm 0.002^{a}$ & $229 \pm 3$ & $31 \pm 1^{a}$ & [S II] \\
\hline & 12 & 55 & 05.4 & -76 & 58 & 01 & $0.009 \pm 0.002$ & $47 \pm 14$ & $8 \pm 2$ & $\mathrm{H} \alpha$ \\
\hline HH 52 C2 & 12 & 55 & 05.5 & -76 & 57 & 58 & $0.056 \pm 0.002^{a}$ & $38 \pm 2$ & $48 \pm 2^{a}$ & [S II] \\
\hline & 12 & 55 & 05.4 & -76 & 57 & 58 & $0.046 \pm 0.004$ & $56 \pm 5$ & $39 \pm 3$ & $\mathrm{H} \alpha$ \\
\hline HH 52 C3 & 12 & 55 & 06.2 & -76 & 57 & 59 & - & - & - & {$[\mathrm{S}$ II $], \mathrm{H} \alpha$} \\
\hline HH 52 C4 & 12 & 55 & 06.7 & -76 & 57 & 58 & $0.056 \pm 0.002^{a}$ & $4 \pm 2$ & $47 \pm 2^{a}$ & [S II] \\
\hline & 12 & 55 & 06.7 & -76 & 57 & 58 & $0.068 \pm 0.003^{a}$ & $4 \pm 3$ & $58 \pm 2^{a}$ & $\mathrm{H} \alpha$ \\
\hline HH 52 C5 & 12 & 55 & 08.0 & -76 & 58 & 01 & - & - & - & [S II] \\
\hline HH 52 C6 & 12 & 55 & 05.3 & -76 & 58 & 01 & - & - & - & {$[\mathrm{S}$ II $], \mathrm{H} \alpha$} \\
\hline HH 52 D1 & 12 & 55 & 13.9 & -76 & 57 & 47 & - & - & - & [S II $]$ \\
\hline & 12 & 55 & 13.9 & -76 & 57 & 47 & $0.060 \pm 0.006$ & $35 \pm 5$ & $50 \pm 5$ & $\mathrm{H} \alpha$ \\
\hline HH 52 D2 & 12 & 55 & 12.8 & -76 & 57 & 49 & $0.046 \pm 0.002$ & $32 \pm 2$ & $38 \pm 2$ & [S II] \\
\hline & 12 & 55 & 12.7 & -76 & 57 & 49 & $0.049 \pm 0.002$ & $23 \pm 3$ & $41 \pm 2$ & $\mathrm{H} \alpha$ \\
\hline HH 52 D3 & 12 & 55 & 12.1 & -76 & 57 & 49 & $0.046 \pm 0.002$ & $49 \pm 2$ & $39 \pm 2$ & [S II] \\
\hline & 12 & 55 & 12.0 & -76 & 57 & 48 & $0.047 \pm 0.003$ & $22 \pm 3$ & $40 \pm 2$ & $\mathrm{H} \alpha$ \\
\hline HH 52 D4 & 12 & 55 & 10.0 & -76 & 57 & 46 & $0.044 \pm 0.002^{a}$ & $47 \pm 2$ & $37 \pm 1^{a}$ & [S II] \\
\hline & 12 & 55 & 09.7 & -76 & 57 & 47 & $0.043 \pm 0.003$ & $57 \pm 4$ & $36 \pm 3$ & $\mathrm{H} \alpha$ \\
\hline HH 52 D5 & 12 & 55 & 06.7 & -76 & 57 & 43 & $0.036 \pm 0.002^{a}$ & $53 \pm 3$ & $30 \pm 2^{a}$ & [S II] \\
\hline & 12 & 55 & 06.6 & -76 & 57 & 44 & $0.040 \pm 0.003$ & $24 \pm 4$ & $34 \pm 3$ & $\mathrm{H} \alpha$ \\
\hline HH 53 A & 12 & 55 & 15.4 & -76 & 57 & 30 & $0.019 \pm 0.002^{a}$ & $303 \pm 5$ & $16 \pm 1^{a}$ & [S II] \\
\hline & 12 & 55 & 15.4 & -76 & 57 & 30 & $0.021 \pm 0.003$ & $275 \pm 6$ & $18 \pm 2$ & $\mathrm{H} \alpha$ \\
\hline HH 53 B & 12 & 55 & 14.5 & -76 & 57 & 31 & $0.007 \pm 0.002^{a}$ & $235 \pm 14$ & $6 \pm 2^{a}$ & [S II] \\
\hline & 12 & 55 & 14.5 & -76 & 57 & 31 & $0.013 \pm 0.003$ & $252 \pm 11$ & $11 \pm 2$ & $\mathrm{H} \alpha$ \\
\hline HH 53 B 1 & 12 & 55 & 13.6 & -76 & 57 & 31 & $0.081 \pm 0.002$ & $262 \pm 1$ & $69 \pm 2$ & $\mathrm{H} \alpha$ \\
\hline НH 53 В2 & 12 & 55 & 14.1 & -76 & 57 & 31 & - & - & - & {$[\mathrm{S}$ II $], \mathrm{H} \alpha$} \\
\hline HH 53 C & 12 & 55 & 16.5 & -76 & 57 & 30 & $0.013 \pm 0.002$ & $239 \pm 9$ & $11 \pm$ & [S II] \\
\hline & 12 & 55 & 16.5 & -76 & 57 & 30 & $0.014 \pm 0.003$ & $232 \pm 12$ & $12 \pm 3$ & $\mathrm{H} \alpha$ \\
\hline HH $53 \mathrm{C} 1$ & 12 & 55 & 16.1 & -76 & 57 & 30 & $0.060 \pm 0.006$ & $58 \pm 6$ & $50 \pm 5$ & $\mathrm{H} \alpha$ \\
\hline HH 53 E & 12 & 55 & 19.4 & -76 & 57 & 21 & $0.016 \pm 0.002$ & $240 \pm 6$ & $14 \pm 2$ & [S II] \\
\hline & 12 & 55 & 19.3 & -76 & 57 & 21 & - & - & - & $\mathrm{H} \alpha$ \\
\hline HH 53 E1 & 12 & 55 & 19.9 & -76 & 57 & 24 & $0.052 \pm 0.004$ & $63 \pm 4$ & $44 \pm 3$ & [S II] \\
\hline & 12 & 55 & 19.8 & -76 & 57 & 24 & $0.049 \pm 0.003$ & $97 \pm 3$ & $41 \pm 3$ & $\mathrm{H} \alpha$ \\
\hline HH 53 F & 12 & 55 & 23.0 & -76 & 57 & 23 & $0.031 \pm 0.002$ & $64 \pm 3$ & $26 \pm 1$ & [S II] \\
\hline & 12 & 55 & 23.0 & -76 & 57 & 23 & $0.049 \pm 0.003$ & $66 \pm 3$ & $41 \pm 3$ & $\mathrm{H} \alpha$ \\
\hline HH 53 F1 & 12 & 55 & 24.6 & -76 & 57 & 20 & $0.079 \pm 0.002^{a}$ & $64 \pm 2$ & $67 \pm 2^{a}$ & [S II] \\
\hline & 12 & 55 & 24.5 & -76 & 57 & 20 & $0.049 \pm 0.003^{a}$ & $89 \pm 3$ & $42 \pm 2^{a}$ & $\mathrm{H} \alpha$ \\
\hline
\end{tabular}


Table B.1. continued.

\begin{tabular}{|c|c|c|c|c|c|c|c|c|c|c|}
\hline \multirow{2}{*}{$\begin{array}{c}\text { Knot ID } \\
\text { HH 53 F2 }\end{array}$} & \multicolumn{3}{|c|}{$\begin{array}{c}\alpha(2000.0) \\
(\mathrm{hms})\end{array}$} & \multicolumn{3}{|c|}{$\begin{array}{c}\delta(2000.0) \\
\left({ }^{\circ},{ }^{\prime \prime}\right)\end{array}$} & \multirow{2}{*}{$\begin{array}{c}\mathrm{PM} \\
\left({ }^{\prime \prime} \mathrm{yr}^{-1}\right) \\
0.051 \pm 0.008\end{array}$} & \multirow{2}{*}{$\begin{array}{c}\text { PA } \\
\left(^{\circ}\right) \\
52 \pm 8\end{array}$} & \multirow{2}{*}{$\begin{array}{c}\begin{array}{c}v_{\tan } \\
\left(\mathrm{km} \mathrm{s}^{-1}\right)\end{array} \\
43 \pm 6\end{array}$} & \multirow{2}{*}{$\begin{array}{c}\text { Filter } \\
\mathrm{H} \alpha\end{array}$} \\
\hline & 12 & 55 & 22.1 & -76 & 57 & 28 & & & & \\
\hline HH 53 G & 12 & 55 & 30.3 & -76 & 57 & 11 & $0.063 \pm 0.002^{a}$ & $67 \pm 2$ & $53 \pm 2^{a}$ & {$[\mathrm{~S} \mathrm{II}]$} \\
\hline & 12 & 55 & 30.3 & -76 & 57 & 11 & $0.037 \pm 0.003^{a}$ & $61 \pm 4$ & $31 \pm 2^{a}$ & $\mathrm{H} \alpha$ \\
\hline HH $53 \mathrm{H}$ & 12 & 55 & 15.4 & -76 & 57 & 25 & - & - & - & {$[\mathrm{S} \mathrm{II}]$} \\
\hline & 12 & 55 & 15.3 & -76 & 57 & 25 & $0.065 \pm 0.004$ & $52 \pm 3$ & $55 \pm$ & $\mathrm{H} \alpha$ \\
\hline HH 53 I & 12 & 55 & 13.1 & -76 & 57 & 34 & $0.063 \pm 0.002$ & $51 \pm 2$ & $53 \pm 2$ & {$[\mathrm{~S} \mathrm{II}]$} \\
\hline & 12 & 55 & 12.2 & -76 & 57 & 36 & $0.066 \pm 0.003$ & $53 \pm 2$ & $56 \pm 2$ & $\mathrm{H} \alpha$ \\
\hline
\end{tabular}

Notes: ${ }^{a}$ mean value, variable in velocity, see text and Table C. $1 ;{ }^{b}$ below $3 \sigma$.

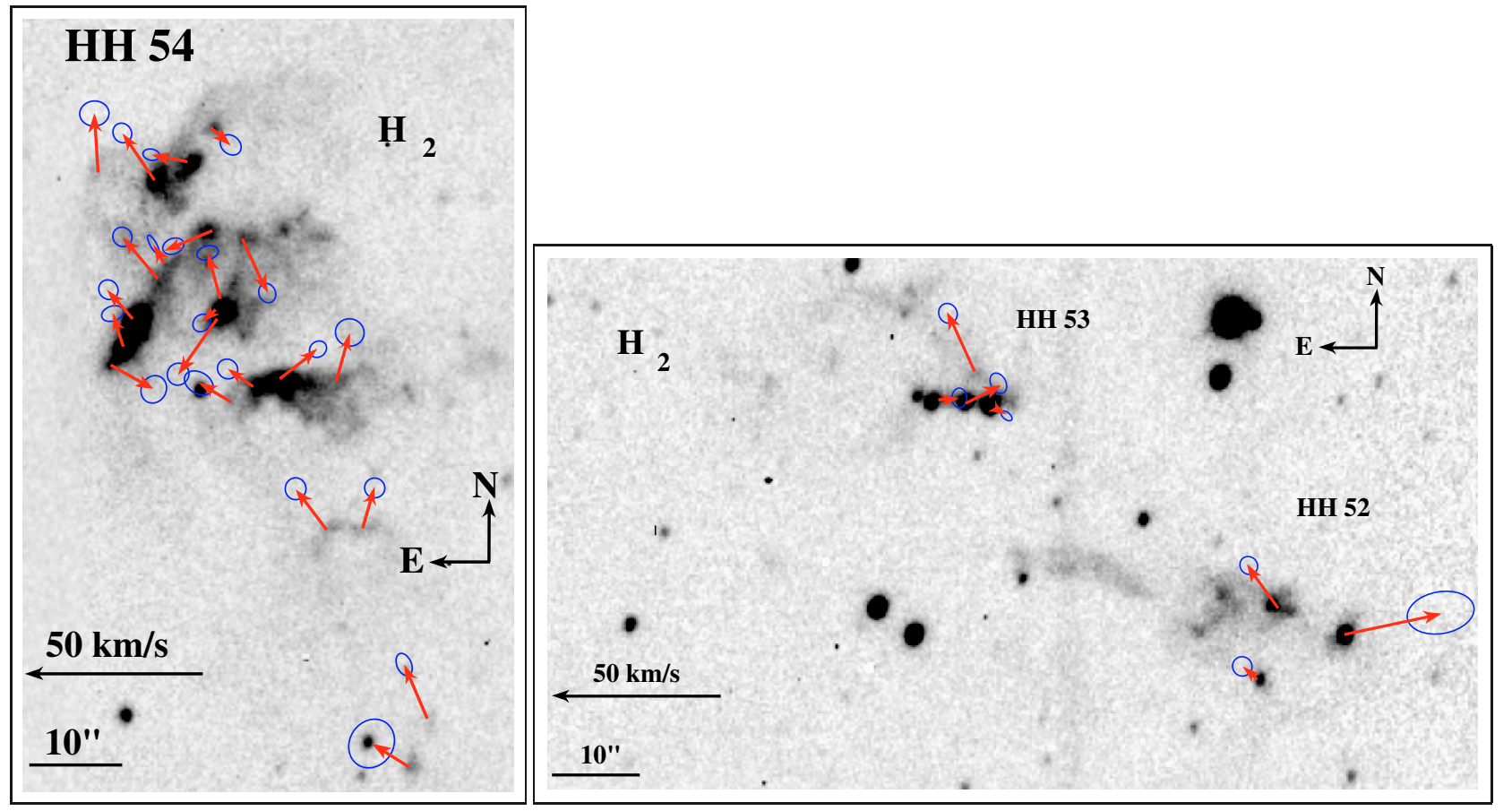

Fig. B.1. Flow charts with error bars of $\mathrm{HH} 54$ (left panel), $\mathrm{HH} 52$ and 53 (right panel) in $\mathrm{H}_{2}$ filter. Proper motions and their error bars are indicated by arrows and ellipses, respectively.

a $\mathrm{PA} \sim 110^{\circ}$, while the second is moving northward (PA $5^{\circ}$ ). A third knot B3, clearly visible from 1989, likely causes the break, proceeding from SE to NE and pushing forward the structure. The direction of the feature considerably changes with time in both optical filters, accordingly, from $\sim 55^{\circ}$ in 1989 to $85^{\circ}$ in 1993 . Also B1 and B2 show variability (see Figs. C.1, 8, and C.2), exhibiting evidence of deceleration and acceleration, respectively.

Knots HH 52 B4-B9, in the outskirts of the wing, exhibit large variability and complicated motions as well. The presence of such a high variability and the large gap between the second to last (1993 for [S II] and 1995 for $\mathrm{H} \alpha$ ) and the last epoch image (2006) produce uncertainties in the identification of the knots and in the PM derivation. A large structure, B5, appears in 1989 (see Fig. 7, top panel and also Fig. C.1), rapidly increasing its brightness and then almost vanishing in 2006, apparently fragmented in several knots (B4-B6, and B8), that move in different directions. The flux variation of the object has different timing in the three filters, with a delay of some years between the flux variations of [S II] and $\mathrm{H} \alpha$, and then between $\mathrm{H} \alpha$ and $\mathrm{H}_{2}$ (Fig. 7, top panel). In [S II] it slightly varies its brightness, reaching a maximum possibly around 1991-1993. In $\mathrm{H} \alpha$ and $\mathrm{H}_{2}$ the variations are more considerable, especially in the molecular component, where the flux increases an order of magnitude during six years.

Knots $\mathrm{C}$ in the left wing of HH 52 present a peculiar behaviour as well. $\mathrm{C} 1$ (see Fig. 7, central panel and Fig. C.3) shows a large variability similar to B5, with a large brightness increase in $\mathrm{H} \alpha$, due to the presence of a fast shock colliding against the target. This is clearly visible in 1993 and $1995 \mathrm{H} \alpha$ images (Fig. C.1), where a high velocity knot (C6) appears behind C1. In the 2006 image the bullet is not visible anymore, but an increase in $\mathrm{C} 1$ brightness is detected, in both atomic emissions. As in B5, also the molecular emission increases with time. Here the increment is not so pronounced as in the previous knot. HH $52 \mathrm{C} 2$, located behind HH 52 nucleus, starts forming in 1989 as a [S II] emission (Fig. 7, bottom panel) and increases its luminosity until 2006. On the other hand the $\mathrm{H} \alpha$ emission is visible above a $3 \sigma$ limit only in the 2006 image.

\section{C.2. $\mathrm{HH} 54$}

\section{C.2.1. $\mathrm{HH} 54$ streamer}

Figure C.4 (bottom panel) displays the peculiar kinematics of HH 54 Y2, located in the middle of the streamer. The proper 
A. Caratti o Garatti et al.: Time-variability in the Chamaeleontis II outflows, Online Material p 6

Table B.2. Proper Motions in HH 54 - H $\alpha$ and [S II] - parameters derived from the linear fit.

\begin{tabular}{|c|c|c|c|c|c|c|c|c|c|c|}
\hline \multirow{2}{*}{$\begin{array}{c}\text { Knot ID } \\
\text { HH 54 A1 }\end{array}$} & \multicolumn{3}{|c|}{$\begin{array}{c}\alpha(2000.0) \\
(\mathrm{hms})\end{array}$} & \multicolumn{3}{|c|}{$\begin{array}{c}\delta(2000.0) \\
\left({ }^{\circ}, \prime \prime\right)\end{array}$} & \multirow{2}{*}{$\begin{array}{c}\mathrm{PM} \\
\left({ }^{\prime \prime} \mathrm{yr}^{-1}\right) \\
0.036 \pm 0.004\end{array}$} & \multirow{2}{*}{$\begin{array}{c}\text { PA } \\
\left({ }^{\circ}\right) \\
70 \pm 6\end{array}$} & \multirow{2}{*}{$\begin{array}{c}\begin{array}{c}v_{\tan } \\
\left(\mathrm{km} \mathrm{s}^{-1}\right)\end{array} \\
31 \pm 3\end{array}$} & \multirow{2}{*}{$\begin{array}{l}\text { Filter } \\
{[\mathrm{S} \text { II }]}\end{array}$} \\
\hline & 12 & 55 & 49.4 & -76 & 56 & 30 & & & & \\
\hline & 12 & 55 & 49.4 & -76 & 56 & 30 & $0.035 \pm 0.002$ & $85 \pm 2$ & $29 \pm 2$ & $\mathrm{H} \alpha$ \\
\hline \multirow[t]{2}{*}{ HH 54 A2 } & 12 & 55 & 50.2 & -76 & 56 & 30 & $0.036 \pm 0.004$ & $81 \pm 6$ & $30 \pm 3$ & [S II] \\
\hline & 12 & 55 & 50.2 & -76 & 56 & 30 & $0.034 \pm 0.002$ & $73 \pm 3$ & $29 \pm 2$ & $\mathrm{H} \alpha$ \\
\hline \multirow[t]{2}{*}{ HH 54 A3 } & 12 & 55 & 48.7 & -76 & 56 & 33 & $0.013 \pm 0.004$ & $166 \pm 17$ & $11 \pm 3$ & [S II] \\
\hline & 12 & 55 & 48.7 & -76 & 56 & 33 & $0.015 \pm 0.002$ & $121 \pm 6$ & $13 \pm 3$ & $\mathrm{H} \alpha$ \\
\hline \multirow[t]{2}{*}{ HH 54 A4 } & 12 & 55 & 47.7 & -76 & 56 & 33 & $0.030 \pm 0.004$ & $225 \pm 7$ & $25 \pm 3$ & [S II $]$ \\
\hline & 12 & 55 & 47.8 & -76 & 56 & 34 & - & - & - & $\mathrm{H} \alpha$ \\
\hline \multirow{2}{*}{ HH 54 A5 } & 12 & 55 & 47.4 & -76 & 56 & 30 & $0.066 \pm 0.006$ & $125 \pm 6$ & $56 \pm 5$ & [S II] \\
\hline & 12 & 55 & 47.4 & -76 & 56 & 31 & - & - & - & $\mathrm{H} \alpha$ \\
\hline HH 54 A6 & 12 & 55 & 48.1 & -76 & 56 & 31 & $0.031 \pm 0.007$ & $53 \pm 13$ & $27 \pm 6$ & [S II] \\
\hline & 12 & 55 & 48.1 & -76 & 56 & 31 & $0.025 \pm 0.005$ & $40 \pm 11$ & $21 \pm 4$ & $\mathrm{H} \alpha$ \\
\hline HH 54 A7 & 12 & 55 & 49.4 & -76 & 56 & 32 & $0.017 \pm 0.005$ & $88 \pm 27$ & $14 \pm 4$ & [S II] \\
\hline & 12 & 55 & 49.4 & -76 & 56 & 32 & $0.016 \pm 0.002$ & $23 \pm 6$ & $14 \pm 1$ & $\mathrm{H} \alpha$ \\
\hline HH 54 A8 & 12 & 55 & 48.7 & -76 & 56 & 29 & $0.039 \pm 0.009$ & $28 \pm 20$ & $33 \pm 8$ & [S II] \\
\hline & 12 & 55 & 48.7 & -76 & 56 & 29 & $0.034 \pm 0.002$ & $66 \pm 3$ & $29 \pm 2$ & $\mathrm{H} \alpha$ \\
\hline НH 54 B & 12 & 55 & 50.9 & -76 & 56 & 21 & $0.008 \pm 0.004^{b}$ & $110 \pm 30$ & $6 \pm 3^{b}$ & [S II] \\
\hline & 12 & 55 & 50.9 & -76 & 56 & 21 & - & - & - & $\mathrm{H} \alpha$ \\
\hline HH 54 B1 & 12 & 55 & 50.6 & -76 & 56 & 19 & $0.010 \pm 0.005^{b}$ & $282 \pm 32$ & $8 \pm 4^{b}$ & [S II] \\
\hline & 12 & 55 & 50.6 & -76 & 56 & 19 & - & - & - & $\mathrm{H} \alpha$ \\
\hline HH 54 B2 & 12 & 55 & 49.8 & -76 & 56 & 21 & - & - & - & [S II] \\
\hline & 12 & 55 & 49.8 & -76 & 56 & 22 & $0.017 \pm 0.002$ & $313 \pm 7$ & $15 \pm 2$ & $\mathrm{H} \alpha$ \\
\hline НH 54 B3 & 12 & 55 & 50.5 & -76 & 56 & 24 & - & - & - & $\mathrm{H} \alpha$ \\
\hline HH 54 C1 & 12 & 55 & 51.3 & -76 & 56 & 05 & $0.032 \pm 0.004$ & $73 \pm 8$ & $27 \pm 3$ & [S II $]$ \\
\hline & 12 & 55 & 51.3 & -76 & 56 & 05 & $0.017 \pm 0.002$ & $67 \pm 5$ & $14 \pm 1$ & $\mathrm{H} \alpha$ \\
\hline HH 54 C2 & 12 & 55 & 50.4 & -76 & 56 & 03 & $0.041 \pm 0.004$ & $42 \pm 5$ & $34 \pm 3$ & [S II] \\
\hline & 12 & 55 & 50.4 & -76 & 56 & 03 & $0.017 \pm 0.002$ & $70 \pm 5$ & $14 \pm 1$ & $\mathrm{H} \alpha$ \\
\hline HH 54 C3 & 12 & 55 & 51.0 & -76 & 56 & 02 & $0.017 \pm 0.006^{b}$ & $239 \pm 19$ & $14 \pm 5^{b}$ & [S II] \\
\hline & 12 & 55 & 51.0 & -76 & 56 & 02 & $0.016 \pm 0.002^{a}$ & $267 \pm 6$ & $13 \pm 2^{a}$ & $\mathrm{H} \alpha$ \\
\hline HH 54 E & 12 & 55 & 53.7 & -76 & 56 & 22 & $0.017 \pm 0.005$ & $35 \pm 15$ & $14 \pm 4$ & [S II $]$ \\
\hline & 12 & 55 & 53.7 & -76 & 56 & 22 & $0.018 \pm 0.002$ & $16 \pm 6$ & $15 \pm 2$ & $\mathrm{H} \alpha$ \\
\hline HH 54 F & 12 & 55 & 49.0 & -76 & 56 & 19 & $0.018 \pm 0.003$ & $125 \pm 11$ & $15 \pm 3$ & [S II] \\
\hline & 12 & 55 & 49.0 & -76 & 56 & 19 & $0.013 \pm 0.002$ & $129 \pm 7$ & $11 \pm 2$ & $\mathrm{H} \alpha$ \\
\hline HH 54 F1 & 12 & 55 & 48.4 & -76 & 56 & 17 & $0.015 \pm 0.002^{a}$ & $251 \pm 9$ & $13 \pm 2^{a}$ & $\mathrm{H} \alpha$ \\
\hline HH 54 G & 12 & 55 & 45.4 & -76 & 56 & 13 & $0.048 \pm 0.004^{a}$ & $78 \pm 4$ & $41 \pm 3^{a}$ & [S II] \\
\hline & 12 & 55 & 45.4 & -76 & 56 & 13 & $0.035 \pm 0.001^{a}$ & $71 \pm 3$ & $29 \pm 1^{a}$ & $\mathrm{H} \alpha$ \\
\hline HH 54 G0 & 12 & 55 & 44.8 & -76 & 56 & 13 & $0.036 \pm 0.005^{a}$ & $36 \pm 7$ & $31 \pm 4^{a}$ & [S II] \\
\hline & 12 & 55 & 44.8 & -76 & 56 & 13 & $0.055 \pm 0.003^{a}$ & $52 \pm 3$ & $46 \pm 3^{a}$ & $\mathrm{H} \alpha$ \\
\hline HH 54 G1 & 12 & 55 & 47.3 & -76 & 56 & 09 & $0.096 \pm 0.006^{a}$ & $81 \pm 3$ & $81 \pm 5^{a}$ & [S II $]$ \\
\hline & 12 & 55 & 47.3 & -76 & 56 & 09 & $0.073 \pm 0.006^{a}$ & $72 \pm 6$ & $62 \pm 5^{a}$ & $\mathrm{H} \alpha$ \\
\hline HH 54 G2 & 12 & 55 & 47.6 & -76 & 56 & 05 & $0.017 \pm 0.005^{b}$ & $58 \pm 17$ & $14 \pm 5^{b}$ & [S II $]$ \\
\hline & 12 & 55 & 47.8 & -76 & 56 & 05 & $0.079 \pm 0.003^{a}$ & $68 \pm 2$ & $67 \pm 2^{a}$ & $\mathrm{H} \alpha$ \\
\hline HH 54 G3 & 12 & 55 & 49.1 & -76 & 56 & 06 & $0.079 \pm 0.006^{a}$ & $63 \pm 4$ & $67 \pm 5^{a}$ & [S II] \\
\hline & 12 & 55 & 49.3 & -76 & 56 & 06 & $0.080 \pm 0.002^{a}$ & $66 \pm 2$ & $68 \pm 2^{a}$ & $\mathrm{H} \alpha$ \\
\hline HH 54 H1 & 12 & 55 & 52.8 & -76 & 56 & 05 & $0.026 \pm 0.004$ & $36 \pm 8$ & $22 \pm 3$ & [S II] \\
\hline & 12 & 55 & 52.8 & -76 & 56 & 05 & $0.033 \pm 0.002^{a}$ & $53 \pm 4$ & $28 \pm 2^{a}$ & $\mathrm{H} \alpha$ \\
\hline HH 54 H2 & 12 & 55 & 53.1 & -76 & 56 & 07 & $0.035 \pm 0.004$ & $23 \pm 6$ & $29 \pm 3$ & [S II] \\
\hline & 12 & 55 & 53.1 & -76 & 56 & 07 & $0.051 \pm 0.002^{a}$ & $38 \pm 3$ & $43 \pm 2^{a}$ & $\mathrm{H} \alpha$ \\
\hline HH 54 H3 & 12 & 55 & 52.4 & -76 & 56 & 10 & $0.025 \pm 0.003$ & $187 \pm 9$ & $21 \pm 3$ & [S II] \\
\hline & 12 & 55 & 52.6 & -76 & 56 & 11 & $0.043 \pm 0.003^{a}$ & $127 \pm 4$ & $37 \pm 3^{a}$ & $\mathrm{H} \alpha$ \\
\hline HH 54 I & 12 & 55 & 54.4 & -76 & 56 & 08 & $0.031 \pm 0.006$ & $48 \pm 11$ & $26 \pm 5$ & [S II] \\
\hline & 12 & 55 & 54.5 & -76 & 56 & 08 & $0.031 \pm 0.002$ & $41 \pm 4$ & $26 \pm 2$ & $\mathrm{H} \alpha$ \\
\hline HH 54 J & 12 & 55 & 52.3 & -76 & 56 & 17 & $0.012 \pm 0.005^{b}$ & $45 \pm 25$ & $10 \pm 4^{b}$ & [S II $]$ \\
\hline & 12 & 55 & 52.3 & -76 & 56 & 18 & - & - & - & {$[\mathrm{S}$ II $], \mathrm{H} \alpha$} \\
\hline HH 54 J1 & 12 & 55 & 51.4 & -76 & 56 & 16 & - & - & - & {$[\mathrm{S} \mathrm{II}], \mathrm{H} \alpha$} \\
\hline HH 54 K & 12 & 55 & 53.8 & -76 & 56 & 25 & $0.017 \pm 0.003$ & $2 \pm 14$ & $14 \pm 3$ & [S II] \\
\hline & 12 & 55 & 53.8 & -76 & 56 & 25 & $0.021 \pm 0.003$ & $329 \pm 11$ & $18 \pm 3$ & $\mathrm{H} \alpha$ \\
\hline HH 54 K1 & 12 & 55 & 54.1 & -76 & 56 & 27 & $0.042 \pm 0.004$ & $207 \pm 5$ & $36 \pm 3$ & [S II] \\
\hline & 12 & 55 & 54.2 & -76 & 56 & 27 & $0.048 \pm 0.005$ & $233 \pm 7$ & $41 \pm 4$ & $\mathrm{H} \alpha$ \\
\hline HH 54 K2 & 12 & 55 & 53.3 & -76 & 56 & 24 & $0.022 \pm 0.005$ & $45 \pm 14$ & $18 \pm 4$ & [S II] \\
\hline & 12 & 55 & 53.4 & -76 & 56 & 24 & $0.016 \pm 0.002$ & $345 \pm 10$ & $13 \pm 1$ & $\mathrm{H} \alpha$ \\
\hline HH 54 M & 12 & 55 & 51.2 & -76 & 56 & 08 & $0.026 \pm 0.004$ & $76 \pm 9$ & $22 \pm 4$ & [S II] \\
\hline & 12 & 55 & 51.0 & -76 & 56 & 09 & $0.019 \pm 0.002^{a}$ & $81 \pm 7$ & $16 \pm 2^{a}$ & $\mathrm{H} \alpha$ \\
\hline HН 54 M1 & 12 & 55 & 50.2 & -76 & 56 & 11 & - & - & - & {$[\mathrm{S}$ II $], \mathrm{H} \alpha$} \\
\hline HH $54 \mathrm{~N}$ & 12 & 55 & 48.8 & -76 & 56 & 23 & $0.029 \pm 0.005$ & $312 \pm 9$ & $25 \pm 4$ & [S II] \\
\hline
\end{tabular}


Table B.2. continued.

\begin{tabular}{|c|c|c|c|c|c|c|c|c|c|c|}
\hline \multirow[t]{2}{*}{$\overline{\text { Knot ID }}$} & \multicolumn{3}{|c|}{$\begin{array}{c}(2000.0) \\
(\mathrm{hms})\end{array}$} & \multicolumn{3}{|c|}{$\begin{array}{c}\delta(2000.0) \\
\left({ }^{\circ},{ }^{\prime} \prime\right)\end{array}$} & \multirow{2}{*}{$\begin{array}{c}\mathrm{PM} \\
\left({ }^{\prime \prime} \mathrm{yr}^{-1}\right) \\
0.021 \pm 0.002\end{array}$} & \multirow{2}{*}{$\begin{array}{c}\text { PA } \\
\left({ }^{\circ}\right) \\
326 \pm 6\end{array}$} & \multirow{2}{*}{$\begin{array}{c}\begin{array}{c}v_{\tan } \\
\left(\mathrm{km} \mathrm{s}^{-1}\right)\end{array} \\
22 \pm 2\end{array}$} & \multirow{2}{*}{$\begin{array}{c}\text { Filter } \\
\mathrm{H} \alpha\end{array}$} \\
\hline & 12 & 55 & 49.0 & -76 & 56 & 23 & & & & \\
\hline \multirow[t]{2}{*}{ HH $54 \mathrm{O}$} & 12 & 55 & 47.4 & -76 & 56 & 22 & $0.013 \pm 0.004$ & $328 \pm 16$ & $11 \pm 3$ & [S II $]$ \\
\hline & 12 & 55 & 47.3 & -76 & 56 & 22 & $0.051 \pm 0.005$ & $58 \pm 6$ & $43 \pm 4$ & $\mathrm{H} \alpha$ \\
\hline \multirow[t]{2}{*}{ HH $54 \mathrm{O} 1$} & 12 & 55 & 46.7 & -76 & 56 & 24 & $0.046 \pm 0.004$ & $15 \pm 7$ & $39 \pm 3$ & [S II $]$ \\
\hline & 12 & 55 & 46.6 & -76 & 56 & 24 & $0.037 \pm 0.003^{a}$ & $47 \pm 5$ & $31 \pm 3^{a}$ & $\mathrm{H} \alpha$ \\
\hline HH 54 P & 12 & 55 & 45.2 & -76 & 56 & 34 & - & - & - & {$[\mathrm{S}$ II $], \mathrm{H} \alpha$} \\
\hline \multirow[t]{2}{*}{ HH 54 Q1 } & 12 & 55 & 47.2 & -76 & 56 & 43 & $0.044 \pm 0.006$ & $23 \pm 8$ & $37 \pm 5$ & [S II] \\
\hline & 12 & 55 & 52.5 & -76 & 56 & 43 & $0.041 \pm 0.002^{a}$ & $37 \pm 3$ & $34 \pm 2^{a}$ & $\mathrm{H} \alpha$ \\
\hline \multirow[t]{2}{*}{ HH 54 Q2 } & 12 & 55 & 51.8 & -76 & 56 & 40 & $0.046 \pm 0.013$ & $34 \pm 17$ & $39 \pm 11$ & [S II $]$ \\
\hline & 12 & 55 & 51.8 & -76 & 56 & 40 & $0.043 \pm 0.003$ & $79 \pm 4$ & $37 \pm 3$ & $\mathrm{H} \alpha$ \\
\hline \multirow[t]{2}{*}{ HH 54 Q3 } & 12 & 55 & 51.3 & -76 & 56 & 36 & - & - & - & {$[\mathrm{S}$ II $]$} \\
\hline & 12 & 55 & 51.3 & -76 & 56 & 36 & $0.043 \pm 0.003^{a}$ & $80 \pm 4$ & $37 \pm 2^{a}$ & $\mathrm{H} \alpha$ \\
\hline HH 54 R & 12 & 55 & 56.3 & -76 & 57 & 20 & - & - & - & {$[\mathrm{S} \mathrm{II}], \mathrm{H} \alpha$} \\
\hline HН 54 X0 & 12 & 55 & 40.3 & -76 & 57 & 50 & $0.022 \pm 0.003$ & $325 \pm 6$ & $19 \pm 2$ & $\mathrm{H} \alpha$ \\
\hline HH 54 X1 A & 12 & 55 & 44.6 & -76 & 57 & 35 & $0.035 \pm 0.003^{a}$ & $101 \pm 4$ & $29 \pm 2^{a}$ & $\mathrm{H} \alpha$ \\
\hline \multirow{2}{*}{ HH 54 X1 B } & 12 & 55 & 42.8 & -76 & 57 & 36 & $0.013 \pm 0.002$ & $313 \pm 11$ & $11 \pm 2$ & [S II] \\
\hline & 12 & 55 & 42.8 & -76 & 57 & 37 & $0.012 \pm 0.003$ & $275 \pm 8$ & $10 \pm 3$ & $\mathrm{H} \alpha$ \\
\hline HH 5 & 12 & 55 & 41.6 & -76 & 57 & 35 & $0.036 \pm 0.002^{a}$ & $340 \pm 4$ & $30 \pm 2^{a}$ & $\mathrm{H} \alpha$ \\
\hline HH 54 X1 D & 12 & 55 & 43.8 & -76 & 57 & 27 & $0.037 \pm 0.004$ & $33 \pm 7$ & $31 \pm 4$ & $\mathrm{H} \alpha$ \\
\hline \multirow[t]{2}{*}{ HH 54 X2 A } & 12 & 55 & 40.7 & -76 & 57 & 42 & $0.018 \pm 0.006$ & $204 \pm 23$ & $15 \pm 5$ & {$[\mathrm{~S}$ II $]$} \\
\hline & 12 & 55 & 40.9 & -76 & 57 & 39 & $0.021 \pm 0.002$ & $55 \pm 6$ & $18 \pm 2$ & $\mathrm{H} \alpha$ \\
\hline \multirow[t]{2}{*}{ HH 54 X2 B } & 12 & 55 & 41.3 & -76 & 57 & 39 & $0.013 \pm 0.002$ & $314 \pm 11$ & $11 \pm 2$ & {$[\mathrm{~S}$ II $]$} \\
\hline & 12 & 55 & 41.0 & -76 & 57 & 41 & $0.036 \pm 0.002$ & $253 \pm 5$ & $30 \pm 2$ & $\mathrm{H} \alpha$ \\
\hline \multirow{2}{*}{ HH 54 X3 } & 12 & 55 & 39.8 & -76 & 57 & 36 & - & - & - & [S II] \\
\hline & 12 & 55 & 39.8 & -76 & 57 & 36 & $0.021 \pm 0.003$ & $256 \pm 13$ & $18 \pm 4$ & $\mathrm{H} \alpha$ \\
\hline HH 54 X4 A & 12 & 55 & 40.5 & -76 & 57 & 28 & $0.068 \pm 0.007$ & $45 \pm 6$ & $57 \pm 7$ & $\mathrm{H} \alpha$ \\
\hline HН 54 X4 B & 12 & 55 & 42.0 & -76 & 57 & 22 & $0.043 \pm 0.003$ & $22 \pm 5$ & $37 \pm 3$ & $\mathrm{H} \alpha$ \\
\hline \multirow{2}{*}{ HH 54 X 4 C } & 12 & 55 & 41.8 & -76 & 57 & 25 & $0.067 \pm 0.006$ & $49 \pm 5$ & $57 \pm 5$ & [S II $]$ \\
\hline & 12 & 55 & 41.8 & -76 & 57 & 25 & $0.047 \pm 0.003$ & $44 \pm 3$ & $39 \pm 2$ & $\mathrm{H} \alpha$ \\
\hline \multirow[t]{2}{*}{ HH 54 Y1 } & 12 & 55 & 44.9 & -76 & 57 & 11 & $0.033 \pm 0.005$ & $33 \pm 8$ & $28 \pm 4$ & [S II] \\
\hline & 12 & 55 & 44.9 & -76 & 57 & 11 & $0.018 \pm 0.002$ & $356 \pm 5$ & $16 \pm 2$ & $\mathrm{H} \alpha$ \\
\hline \multirow[t]{2}{*}{ HH 54 Y2 } & 12 & 55 & 44.7 & -76 & 57 & 06 & $0.038 \pm 0.005$ & $18 \pm 9$ & 32 & {$[\mathrm{~S}$ II $]$} \\
\hline & 12 & 55 & 44.6 & -76 & 57 & 07 & $0.037 \pm 0.002^{a}$ & $26 \pm 3$ & $31 \pm 2^{a}$ & $\mathrm{H} \alpha$ \\
\hline \multirow[t]{2}{*}{ HH 54 Y3 } & 12 & 55 & 43.2 & -76 & 57 & 13 & $0.042 \pm 0.004$ & $63 \pm 5$ & $36 \pm 4$ & {$[\mathrm{~S}$ II $]$} \\
\hline & 12 & 55 & 43.2 & -76 & 57 & 13 & $0.068 \pm 0.003^{a}$ & $43 \pm 3$ & $58 \pm 3$ & $\mathrm{H} \alpha$ \\
\hline НH 54 Y4 & 12 & 55 & 45.0 & -76 & 57 & 00 & $0.043 \pm 0.008$ & $62 \pm 17$ & $36 \pm 7$ & {$[\mathrm{~S}$ II $]$} \\
\hline & 12 & 55 & 45.0 & -76 & 57 & 00 & $0.067 \pm 0.008$ & $41 \pm 7$ & $57 \pm 7$ & $\mathrm{H} \alpha$ \\
\hline НH 54 Y5 & 12 & 55 & 49.1 & -76 & 57 & 04 & $0.013 \pm 0.004^{b}$ & $14 \pm 19$ & $11 \pm 3^{b}$ & [S II] \\
\hline & 12 & 55 & 49.0 & -76 & 57 & 05 & $0.022 \pm 0.008^{b}$ & $90 \pm 20$ & $19 \pm 7^{b}$ & $\mathrm{H} \alpha$ \\
\hline HH 54 Y6 & 12 & 55 & 45.5 & -76 & 56 & 53 & $0.059 \pm 0.006$ & $69 \pm 6$ & $50 \pm 5$ & {$[\mathrm{~S}$ II $]$} \\
\hline & 12 & 55 & 45.5 & -76 & 56 & 53 & $0.059 \pm 0.010$ & $16 \pm 18$ & $50 \pm 9$ & $\mathrm{H} \alpha$ \\
\hline НH 54 Y7 & 12 & 55 & 46.1 & -76 & 57 & 00 & $0.072 \pm 0.017$ & $322 \pm 15$ & $61 \pm 14$ & $\mathrm{H} \alpha$ \\
\hline НH 54 Y8 & 12 & 55 & 45.3 & -76 & 57 & 09 & $0.026 \pm 0.010^{b}$ & $22 \pm 11$ & $22 \pm 8^{b}$ & $\mathrm{H} \alpha$ \\
\hline НH 54 Z & 12 & 55 & 47.2 & -76 & 56 & 43 & $0.037 \pm 0.004$ & $344 \pm 7$ & $31 \pm 3$ & {$[\mathrm{~S}$ II $]$} \\
\hline & 12 & 55 & 47.2 & -76 & 56 & 45 & $0.010 \pm 0.002$ & $325 \pm 13$ & $8 \pm 2$ & $\mathrm{H} \alpha$ \\
\hline НH 54 Z1 & 12 & 55 & 46.1 & -76 & 56 & 45 & $0.013 \pm 0.002$ & $292 \pm 9$ & $11 \pm 2$ & $\mathrm{H} \alpha$ \\
\hline
\end{tabular}

Notes: ${ }^{a}$ mean value, variable in velocity, see text and Table C. $1 ;{ }^{b}$ below $3 \sigma$.

Table B.3. Proper Motions in $\mathrm{HH} 52$ and $\mathrm{HH} 53-\mathrm{H}_{2}$.

\begin{tabular}{|c|c|c|c|c|c|c|c|c|c|c|}
\hline$\overline{\text { Knot ID }}$ & \multicolumn{3}{|c|}{$\begin{array}{c}\alpha(2000.0) \\
(\mathrm{hms})\end{array}$} & \multicolumn{3}{|c|}{$\begin{array}{c}\delta(2000.0) \\
\left({ }^{\circ},{ }^{\prime \prime}\right)\end{array}$} & $\begin{array}{c}\mathrm{PM} \\
\left({ }^{\prime \prime} \mathrm{yr}^{-1}\right)\end{array}$ & $\begin{array}{l}\mathrm{PA} \\
\left({ }^{\circ}\right)\end{array}$ & $\begin{array}{c}v_{\tan } \\
\left(\mathrm{km} \mathrm{s}^{-1}\right)\end{array}$ & Filter \\
\hline HH 52 B2/B3 & 12 & 55 & 05.0 & -76 & 57 & 54 & $0.026 \pm 0.004^{a}$ & $40 \pm 9$ & $22 \pm 4^{a}$ & $\mathrm{H}_{2}$ \\
\hline HН 52 B5/B6 & 12 & 55 & 02.7 & -76 & 57 & 57 & $0.041 \pm 0.014^{b}$ & $281 \pm 12$ & $35 \pm 12^{b}$ & $\mathrm{H}_{2}$ \\
\hline HH $52 \mathrm{C} 1$ & 12 & 55 & 05.4 & -76 & 58 & 01 & $0.008 \pm 0.004^{b}$ & $51 \pm 26$ & $7 \pm 3^{b}$ & $\mathrm{H}_{2}$ \\
\hline HH 53 A & 12 & 55 & 15.4 & -76 & 57 & 30 & $0.016 \pm 0.003$ & $298 \pm 15$ & $14 \pm 3$ & $\mathrm{H}_{2}$ \\
\hline HН 53 B & 12 & 55 & 14.5 & -76 & 57 & 31 & $0.007 \pm 0.003^{b}$ & $231 \pm 34$ & $6 \pm 3^{b}$ & $\mathrm{H}_{2}$ \\
\hline HH 53 C & 12 & 55 & 16.5 & -76 & 57 & 30 & $0.010 \pm 0.003$ & $270 \pm 26$ & $9 \pm 2$ & $\mathrm{H}_{2}$ \\
\hline HH $53 \mathrm{H}$ & 12 & 55 & 15.2 & -76 & 57 & 26 & $0.024 \pm 0.004$ & $27 \pm 8$ & $21 \pm 4$ & $\mathrm{H}_{2}$ \\
\hline
\end{tabular}

Notes: ${ }^{a}$ mean value, variable in velocity, see text and Table C. $1 ;{ }^{b}$ below $3 \sigma$. 
A. Caratti o Garatti et al.: Time-variability in the Chamaeleontis II outflows, Online Material $p 8$

Table B.4. Proper Motions in $\mathrm{HH} 54-\mathrm{H}_{2}$.

\begin{tabular}{|c|c|c|c|c|c|c|c|c|c|c|}
\hline \multirow{2}{*}{$\begin{array}{l}\text { Knot ID } \\
\text { HH } 54 \mathrm{~A}_{1}\end{array}$} & \multicolumn{3}{|c|}{$\begin{array}{c}\alpha(2000.0) \\
\left({ }^{\mathrm{hms}}\right)\end{array}$} & \multicolumn{3}{|c|}{$\begin{array}{c}\delta(2000.0) \\
\left({ }^{\circ}, \prime \prime\right)\end{array}$} & \multirow{2}{*}{$\begin{array}{c}\mathrm{PM} \\
\left({ }^{\prime \prime} \mathrm{yr}^{-1}\right)\end{array}$} & \multirow{2}{*}{$\begin{array}{c}\text { PA } \\
\left(^{\circ}\right) \\
343 \pm 16\end{array}$} & \multirow{2}{*}{$\begin{array}{c}\begin{array}{c}v_{\tan } \\
\left(\mathrm{km} \mathrm{s}^{-1}\right)\end{array} \\
15 \pm 4\end{array}$} & \multirow{2}{*}{$\begin{array}{c}\text { Filter } \\
\mathrm{H}_{2}\end{array}$} \\
\hline & 12 & 55 & 47.3 & -76 & 56 & 29 & & & & \\
\hline $\mathrm{HH} 54 \mathrm{~A}_{2}$ & 12 & 55 & 50.0 & -76 & 56 & 29 & $0.011 \pm 0.003$ & $51 \pm 17$ & $9 \pm 3$ & $\mathrm{H}_{2}$ \\
\hline $\mathrm{HH} 54 \mathrm{~A}_{3}$ & 12 & 55 & 49.2 & -76 & 56 & 29 & $0.018 \pm 0.004$ & $300 \pm 10$ & $16 \pm 3$ & $\mathrm{H}_{2}$ \\
\hline $\mathrm{HH} 54 \mathrm{~A}_{4}$ & 12 & 55 & 50.9 & -76 & 56 & 30 & $0.014 \pm 0.006^{b}$ & $62 \pm 16$ & $12 \pm 5^{b}$ & $\mathrm{H}_{2}$ \\
\hline HH $54 \mathrm{~B}_{1}$ & 12 & 55 & 51.0 & -76 & 56 & 20 & $0.016 \pm 0.003^{a}$ & $15 \pm 13$ & $14 \pm 2^{a}$ & $\mathrm{H}_{2}$ \\
\hline HH $54 \mathrm{~B}_{2}$ & 12 & 55 & 51.1 & -76 & 56 & 21 & $0.008 \pm 0.003^{b}$ & $131 \pm 23$ & $6 \pm 3^{b}$ & $\mathrm{H}_{2}$ \\
\hline $\mathrm{HH} 54 \mathrm{~B}_{3}$ & 12 & 55 & 51.2 & -76 & 56 & 22 & $0.022 \pm 0.003^{a}$ & $140 \pm 9$ & $18 \pm 3^{a}$ & $\mathrm{H}_{2}$ \\
\hline HH 54 C1 & 12 & 55 & 51.9 & -76 & 56 & 05 & $0.015 \pm 0.003$ & $77 \pm 9$ & $13 \pm 3$ & $\mathrm{H}_{2}$ \\
\hline HH 54 C3 & 12 & 55 & 51.3 & -76 & 56 & 01 & $0.009 \pm 0.004^{b}$ & $239 \pm 19$ & $8 \pm 3^{b}$ & $\mathrm{H}_{2}$ \\
\hline HН 54 E & 12 & 55 & 53.9 & -76 & 56 & 22 & $0.012 \pm 0.003^{a}$ & $48 \pm 14$ & $11 \pm 3$ & $\mathrm{H}_{2}$ \\
\hline НH 54 H2 & 12 & 55 & 53.2 & -76 & 56 & 07 & $0.020 \pm 0.003$ & $39 \pm 9$ & $17 \pm 3$ & $\mathrm{H}_{2}$ \\
\hline HH 54 I & 12 & 55 & 55.2 & -76 & 56 & 06 & $0.016 \pm 0.004^{a}$ & $3 \pm 14$ & $14 \pm 3^{a}$ & $\mathrm{H}_{2}$ \\
\hline HH 54 J & 12 & 55 & 53.0 & -76 & 56 & 17 & $0.019 \pm 0.003$ & $45 \pm 10$ & $16 \pm 3$ & $\mathrm{H}_{2}$ \\
\hline HH 54 J1 & 12 & 55 & 52.6 & -76 & 56 & 16 & $0.008 \pm 0.004^{b}$ & $32 \pm 36$ & $8 \pm 4^{b}$ & $\mathrm{H}_{2}$ \\
\hline HH 54 K & 12 & 55 & 54.1 & -76 & 56 & 25 & $0.012 \pm 0.003$ & $19 \pm 17$ & $10 \pm 2$ & $\mathrm{H}_{2}$ \\
\hline HH 54 K1 & 12 & 55 & 54.6 & -76 & 56 & 27 & $0.019 \pm 0.005$ & $243 \pm 16$ & $16 \pm 4$ & $\mathrm{H}_{2}$ \\
\hline HH 54 M & 12 & 55 & 51.5 & -76 & 56 & 12 & $0.020 \pm 0.007^{b}$ & $110 \pm 13$ & $17 \pm 6^{b}$ & $\mathrm{H}_{2}$ \\
\hline HH 54 M1 & 12 & 55 & 50.3 & -76 & 56 & 13 & $0.021 \pm 0.003$ & $211 \pm 8$ & $18 \pm 3$ & $\mathrm{H}_{2}$ \\
\hline НH 54 T & 12 & 55 & 41.6 & -76 & 57 & 34 & - & - & - & $\mathrm{H}_{2}$ \\
\hline НH 54 Y1 & 12 & 55 & 45.0 & -76 & 57 & 10 & $0.016 \pm 0.008^{b}$ & $67 \pm 25$ & $14 \pm 7^{b}$ & $\mathrm{H}_{2}$ \\
\hline HH 54 Y2 & 12 & 55 & 44.4 & -76 & 57 & 06 & $0.020 \pm 0.005^{a}$ & $21 \pm 7$ & $17 \pm 4^{a}$ & $\mathrm{H}_{2}$ \\
\hline HH 54 Z & 12 & 55 & 47.5 & -76 & 56 & 45 & $0.018 \pm 0.004$ & $39 \pm 12$ & $15 \pm 3$ & $\mathrm{H}_{2}$ \\
\hline НH 54 Z1 & 12 & 55 & 46.3 & -76 & 56 & 45 & $0.015 \pm 0.004$ & $317 \pm 13$ & $13 \pm 3$ & $\mathrm{H}_{2}$ \\
\hline
\end{tabular}

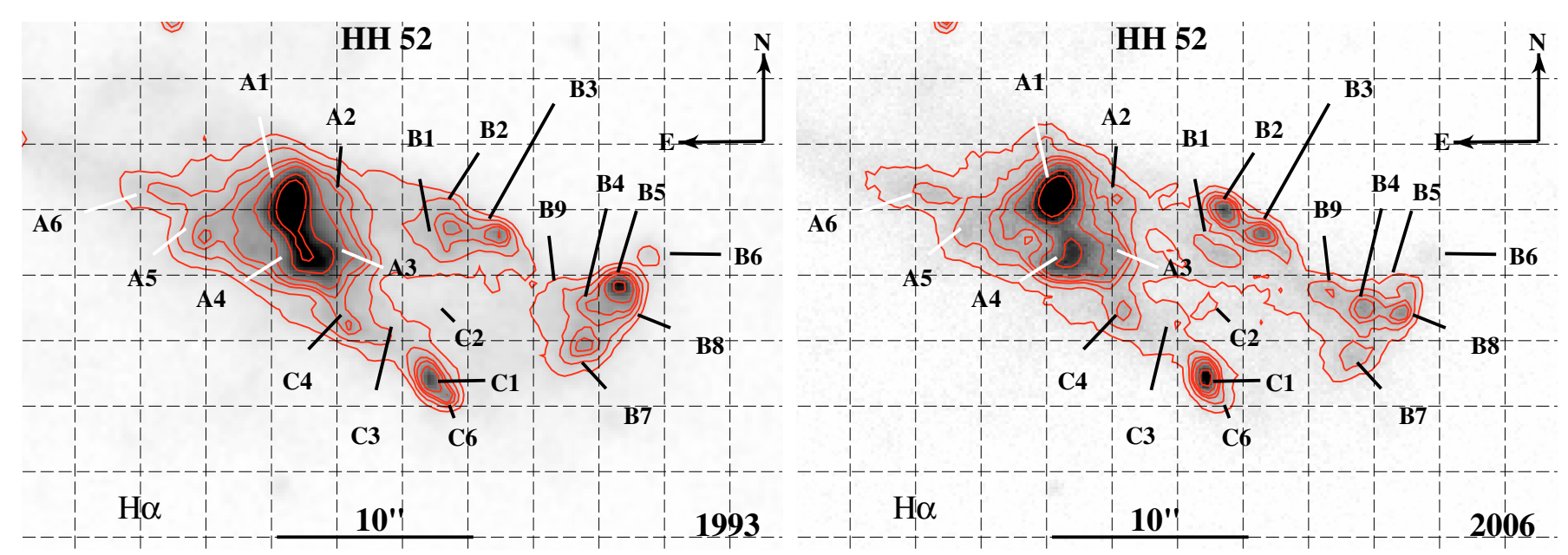

Fig. C.1. Variability in morphology and flux of HH 52. Left panel: close up of HH 52 from 1993 H $\alpha$ calibrated image (labels refer to 2006 image). Right panel: close up of HH 52 from $2006 \mathrm{H} \alpha$ calibrated image. Both images have the same contour levels.

motion measurements reveal an apparent acceleration of the knot, detected in all three filters. However, due to the faint emission, errors in $\mathrm{H}_{2}$ appear remarkably larger. Here, the PM measurements are referred to epoch 2002, since in $\mathrm{H}_{2} 2005$ image $\mathrm{Y} 2$ is out of the FoV. We measure an acceleration of $0.006 \pm$ $0.0002^{\prime \prime} \mathrm{yr}^{-2}$ and $0.01 \pm 0.004^{\prime \prime} \mathrm{yr}^{-2}$ in $\mathrm{H} \alpha$ and $\mathrm{H}_{2}$, respectively (see Table C.1). The average value obtained including both optical $(\mathrm{H} \alpha$ and $[\mathrm{S} \mathrm{II}])$ and molecular PMs is $0.0031 \pm$ $0.0008^{\prime \prime} \mathrm{yr}^{-2}$. Thus a mechanism must exist there that is accelerating the streamer or, conversely, the observed motion could be a reminiscence of the original acceleration experienced by the flow. The upper panel of Fig. C.4 reports the measured flux for the three filters. For this object, all the emissions appear almost constant with time (inside the error bars). This is indeed due to the high degree of ionisation along the HH 54 streamer.

Also along the streamer it is possible to observe the formation of new condensations. A new $\mathrm{H} \alpha$ knot is forming $\sim 1$ ". 6 ENE of HH 54 Y3. This is probably an instability in the flow created by the material compressed and pushed ahead of Y3.

\section{C.2.2. $\mathrm{HH} 54 \mathrm{~A}$}

Figure C.5 illustrates flux variability in HH 54 A group. Here, at variance with other knots, the measured velocities are constant 
A. Caratti o Garatti et al.: Time-variability in the Chamaeleontis II outflows, Online Material p 9

Table C.1. Accelerated Motions in $\mathrm{HH} 52, \mathrm{HH} 53$, and $\mathrm{HH} 54-\mathrm{H} \alpha$, [S II] and $\mathrm{H}_{2}$ - parameters derived from the quadratic fit.

\begin{tabular}{|c|c|c|c|c|c|c|c|}
\hline Knot ID & $\begin{array}{l}P_{a}\left(\chi^{2} / v\right) \\
\times 100(\%) \\
\end{array}$ & $\begin{array}{c}\mathrm{PM} \\
\left(\mathrm{yr}^{-1}\right)\end{array}$ & $\begin{array}{c}\text { Accelerated motion } \\
\left(" \mathrm{yr}^{-2}\right)\end{array}$ & $\begin{array}{c}\mathrm{PA}(\boldsymbol{v}) \\
\left({ }^{\circ}\right)\end{array}$ & $\begin{array}{c}\mathrm{PA}(a) \\
\left({ }^{\circ}\right)\end{array}$ & Filter & $\begin{array}{c}\text { Note } \\
\text { Reliable }\end{array}$ \\
\hline \multirow[t]{2}{*}{ HH 52 A5 } & 0.10 & $0.071 \pm 0.012$ & $0.0067 \pm 0.0013$ & $110 \pm 8$ & $133 \pm 11$ & {$[\mathrm{~S} \mathrm{III}]$} & no \\
\hline & $1.00^{a}$ & $0.137 \pm 0.086^{b}$ & $0.018 \pm 0.008^{b}$ & $5 \pm 33$ & $340 \pm 26$ & $\mathrm{H} \alpha$ & \\
\hline \multirow{2}{*}{ HH 52 B 1} & 0.05 & $0.132 \pm 0.017$ & $0.0226 \pm 0.0018$ & $116 \pm 8$ & $299 \pm 5$ & {$[\mathrm{~S}$ II $]$} & yes \\
\hline & $1.00^{a}$ & $0.093 \pm 0.068^{b}$ & $0.02 \pm 0.01^{b}$ & $111 \pm 20$ & $285 \pm 16$ & $\mathrm{H} \alpha$ & \\
\hline \multirow[t]{2}{*}{ HH 52 B2 } & 0.99 & $0.099 \pm 0.021$ & $0.0060 \pm 0.0021^{b}$ & $7 \pm 11$ & $12 \pm 23$ & [S II] & yes \\
\hline & 1.00 & $0.131 \pm 0.023$ & $0.0109 \pm 0.0027$ & $9 \pm 16$ & $16 \pm 23$ & $\mathrm{H} \alpha$ & \\
\hline НH 52 B3 & 0.34 & $0.122 \pm 0.017$ & $0.0121 \pm 0.0019$ & $112 \pm 7$ & $128 \pm 9$ & {$[\mathrm{~S}$ II $]$} & no \\
\hline НH 52 B2/B3 & $1.00^{a}$ & $0.194 \pm 0.077^{b}$ & $0.061 \pm 0.015$ & $357 \pm 22$ & $348 \pm 23$ & $\mathrm{H}_{2}$ & no \\
\hline НH 52 B 4 & 0.22 & $0.124 \pm 0.016$ & $0.0134 \pm 0.0019$ & $90 \pm 6$ & $94 \pm 7$ & {$[\mathrm{~S}$ II $]$} & no \\
\hline \multirow[t]{2}{*}{ HH 52 B5 } & 0.05 & $0.106 \pm 0.041^{b}$ & $0.0117 \pm 0.0030$ & $243 \pm 13$ & $238 \pm 13$ & {$[\mathrm{~S}$ II $]$} & no \\
\hline & 0.10 & $0.146 \pm 0.045$ & $0.0076 \pm 0.0052^{b}$ & $202 \pm 12$ & $215 \pm 36$ & $\mathrm{H} \alpha$ & \\
\hline НH 52 B 8 & 1.00 & $0.164 \pm 0.067^{b}$ & $0.020 \pm 0.007^{b}$ & $290 \pm 16$ & $291 \pm 19$ & {$[\mathrm{~S}$ II $]$} & yes \\
\hline HH $52 \mathrm{C} 1$ & 0.05 & $0.052 \pm 0.012$ & $0.0105 \pm 0.0014$ & $69 \pm 13$ & $229 \pm 10$ & {$[\mathrm{~S}$ II $]$} & yes \\
\hline \multirow[t]{2}{*}{ HH $52 \mathrm{C} 4$} & 0.28 & $0.144 \pm 0.025$ & $0.0105 \pm 0.0018$ & $347 \pm 7$ & $338 \pm 11$ & [S II] & yes \\
\hline & $1.00^{a}$ & $0.166 \pm 0.047$ & $0.0163 \pm 0.0050^{b}$ & $348 \pm 14$ & $338 \pm 21$ & $\mathrm{H} \alpha$ & \\
\hline \multirow[t]{2}{*}{ HH 52 D4 } & 0.18 & $0.073 \pm 0.014$ & $0.0038 \pm 0.0013^{b}$ & $33 \pm 9$ & $16 \pm 21$ & {$[\mathrm{~S}$ II $]$} & no \\
\hline & 0.53 & $0.093 \pm 0.050^{b}$ & $0.011 \pm 0.0050^{b}$ & $12 \pm 22$ & $347 \pm 28$ & $\mathrm{H} \alpha$ & \\
\hline HH 53 A & 0.93 & $0.034 \pm 0.012^{b}$ & $0.0040 \pm 0.0012$ & $230 \pm 18$ & $197 \pm 20$ & [S II $]$ & no \\
\hline НH 53 B & 0.96 & $0.038 \pm 0.022^{b}$ & $0.0047 \pm 0.0012$ & $332 \pm 18$ & $343 \pm 18$ & {$[\mathrm{~S}$ II $]$} & no \\
\hline \multirow[t]{2}{*}{ HH 53 G } & 0.07 & $0.039 \pm 0.013$ & $0.0049 \pm 0.0014$ & $104 \pm 16$ & $212 \pm 18$ & {$[\mathrm{~S}$ II $]$} & no \\
\hline & $1.00^{a}$ & $0.212 \pm 0.069^{b}$ & $0.0276 \pm 0.0089$ & $105 \pm 15$ & $113 \pm 18$ & $\mathrm{H} \alpha$ & \\
\hline HH $54 \mathrm{~B}_{1}$ & 0.34 & $0.04 \pm 0.01$ & $0.0088 \pm 0.0024$ & $40 \pm 12$ & $61 \pm 15$ & $\mathrm{H}_{2}$ & no \\
\hline $\mathrm{HH} 54 \mathrm{~B}_{3}$ & 0.58 & $0.067 \pm 0.013$ & $0.0145 \pm 0.0023$ & $173 \pm 8$ & $185 \pm 10$ & $\mathrm{H}_{2}$ & no \\
\hline HH 54 C3 & 0.85 & $0.046 \pm 0.020^{b}$ & $0.054 \pm 0.014$ & $184 \pm 21$ & $165 \pm 20$ & $\mathrm{H} \alpha$ & no \\
\hline HH 54 E & 0.86 & $0.027 \pm 0.008$ & $0.0039 \pm 0.0015^{b}$ & $28 \pm 19$ & $16 \pm 41$ & $\mathrm{H}_{2}$ & yes \\
\hline HH 54 F1 & 0.09 & $0.072 \pm 0.024$ & $0.084 \pm 0.019$ & $312 \pm 12$ & $324 \pm 13$ & $\mathrm{H} \alpha$ & no \\
\hline \multirow[t]{2}{*}{ HH 54 G } & $1.00^{a}$ & $0.134 \pm 0.057^{b}$ & $0.013 \pm 0.006^{b}$ & $67 \pm 21$ & $61 \pm 33$ & [S II] & yes \\
\hline & 0.08 & $0.155 \pm 0.015$ & $0.0146 \pm 0.0017$ & $85 \pm 7$ & $89 \pm 9$ & $\mathrm{H} \alpha$ & \\
\hline \multirow[t]{2}{*}{ HH 54 G0 } & $1.00^{a}$ & $0.2 \pm 0.1^{b}$ & $0.020 \pm 0.007^{b}$ & $28 \pm 16$ & $220 \pm 20$ & [S II] & yes \\
\hline & 0.12 & $0.05 \pm 0.02^{b}$ & $0.012 \pm 0.002$ & $40 \pm 20$ & $230 \pm 13$ & $\mathrm{H} \alpha$ & \\
\hline \multirow[t]{2}{*}{ HH 54 G1 } & $1.00^{a}$ & $0.239 \pm 0.067$ & $0.02 \pm 0.009^{b}$ & $79 \pm 15$ & $76 \pm 27$ & [S II] & yes \\
\hline & 0.06 & $0.214 \pm 0.031$ & $0.0177 \pm 0.0039$ & $85 \pm 12$ & $85 \pm 19$ & $\mathrm{H} \alpha$ & \\
\hline \multirow[t]{2}{*}{ HH 54 G2 } & $1.00^{a}$ & $0.12 \pm 0.11^{b}$ & $0.017 \pm 0.0077^{b}$ & $70 \pm 35$ & $316 \pm 26$ & [S II] & no \\
\hline & 0.37 & $0.125 \pm 0.019$ & $0.0097 \pm 0.0026$ & $70 \pm 13$ & $143 \pm 19$ & $\mathrm{H} \alpha$ & \\
\hline \multirow[t]{2}{*}{ HH 54 G3 } & $1.00^{a}$ & $0.291 \pm 0.059$ & $0.0299 \pm 0.0077$ & $76 \pm 11$ & $80 \pm 15$ & {$[\mathrm{~S}$ II $]$} & yes \\
\hline & 0.10 & $0.134 \pm 0.025$ & $0.0068 \pm 0.0025^{b}$ & $66 \pm 16$ & $67 \pm 38$ & $\mathrm{H} \alpha$ & \\
\hline HH 54 H1 & 0.49 & $0.033 \pm 0.013^{b}$ & $0.0063 \pm 0.0017$ & $55 \pm 30$ & $199 \pm 17$ & $\mathrm{H} \alpha$ & yes \\
\hline HH 54 H2 & 0.10 & $0.158 \pm 0.017$ & $0.0228 \pm 0.0019$ & $41 \pm 5$ & $256 \pm 4$ & $\mathrm{H} \alpha$ & yes \\
\hline \multirow[t]{2}{*}{ HH 54 H3 } & $1.00^{a}$ & $0.062 \pm 0.023^{b}$ & $0.012 \pm 0.004$ & $127 \pm 4$ & $273 \pm 16$ & $\mathrm{H} \alpha$ & no \\
\hline & 0.50 & $0.079 \pm 0.017$ & $0.007 \pm 0.003^{b}$ & $66 \pm 12$ & $140 \pm 35$ & $\mathrm{H}_{2}$ & \\
\hline HH 54 M & 1.00 & $0.071 \pm 0.019$ & $0.069 \pm 0.021$ & $70 \pm 14$ & $67 \pm 19$ & $\mathrm{H} \alpha$ & yes \\
\hline \multirow[t]{2}{*}{ HH 54 Q1 } & $1.00^{a}$ & $0.165 \pm 0.103^{b}$ & $0.0171 \pm 0.0078^{b}$ & $12 \pm 20$ & $8 \pm 28$ & [S II] & yes \\
\hline & 0.97 & $0.093 \pm 0.023$ & $0.0084 \pm 0.0023$ & $7 \pm 22$ & $349 \pm 30$ & $\mathrm{H} \alpha$ & \\
\hline HH 54 Q3 & 0.99 & $0.097 \pm 0.018$ & $0.0069 \pm 0.023$ & $79 \pm 12$ & $79 \pm 22$ & $\mathrm{H} \alpha$ & yes \\
\hline HH 54 X1 A & 0.57 & $0.079 \pm 0.015$ & $0.055 \pm 0.018$ & $105 \pm 9$ & $107 \pm 16$ & $\mathrm{H} \alpha$ & yes \\
\hline HH 54 X 1 C & 0.38 & $0.025 \pm 0.012^{b}$ & $0.0065 \pm 0.0015$ & $225 \pm 30$ & $186 \pm 14$ & $\mathrm{H} \alpha$ & no \\
\hline \multirow[t]{2}{*}{ HH 54 Y2 } & 1.00 & $0.082 \pm 0.014$ & $0.0057 \pm 0.0013$ & $25 \pm 7$ & $23 \pm 12$ & $\mathrm{H} \alpha$ & yes \\
\hline & 0.59 & $0.071 \pm 0.026^{b}$ & $0.0098 \pm 0.0037^{b}$ & $19 \pm 16$ & $40 \pm 20$ & $\mathrm{H}_{2}$ & \\
\hline HH 54 Y3 & 0.06 & $0.145 \pm 0.026$ & $0.0097 \pm 0.0026$ & $37 \pm 9$ & $32 \pm 18$ & $\mathrm{H} \alpha$ & yes \\
\hline
\end{tabular}

Notes: ${ }^{a}$ only 3 points in the fit; ${ }^{b}$ below $3 \sigma$.

(inside the error bars) in all the three filters. The brightest feature (knot A1, bottom panel) doubles its $\mathrm{H} \alpha$ luminosity, after 1990. Variability is, however, not observed in [S II], which appears constant during the considered period. The molecular emission, that does not spatially coincide with the atomic one but it is located about 1". 6 forward, from 1993 shows an increase with time. Knot A8, next to A1 along the flow, appears in $\mathrm{H} \alpha$ images in 1989 (Fig. C.5, top panel), and increases its brightness in all the three filters, and it is extremely luminous in the molecular component. In both [S II] and $\mathrm{H}_{2}$ it has a diffuse emission, probably originating from moving gas cooling behind the
$\mathrm{H} \alpha$ emission. The feature has a different direction $\left(\mathrm{PA} \sim 35^{\circ}\right)$ with respect to the bulk of the flow $\left(\mathrm{PA} \sim 80^{\circ}\right)$. This deflection could be generated in the interaction with knot A6 (see also Fig. A.2), a faint emission following the group, that seems to push aside A8, or, conversely, it could indicate that the knot is not part of the same flow.

\section{C.2.3. $\mathrm{HH} 54 \mathrm{G}$}

A few arcseconds ahead of knots G and G0 (see Sect. 4.4), also knots HH 54 G1 and G3 are deflected $\left(\mathrm{PA} \sim 60-80^{\circ}\right)$ and 
A. Caratti o Garatti et al.: Time-variability in the Chamaeleontis II outflows, Online Material p 10

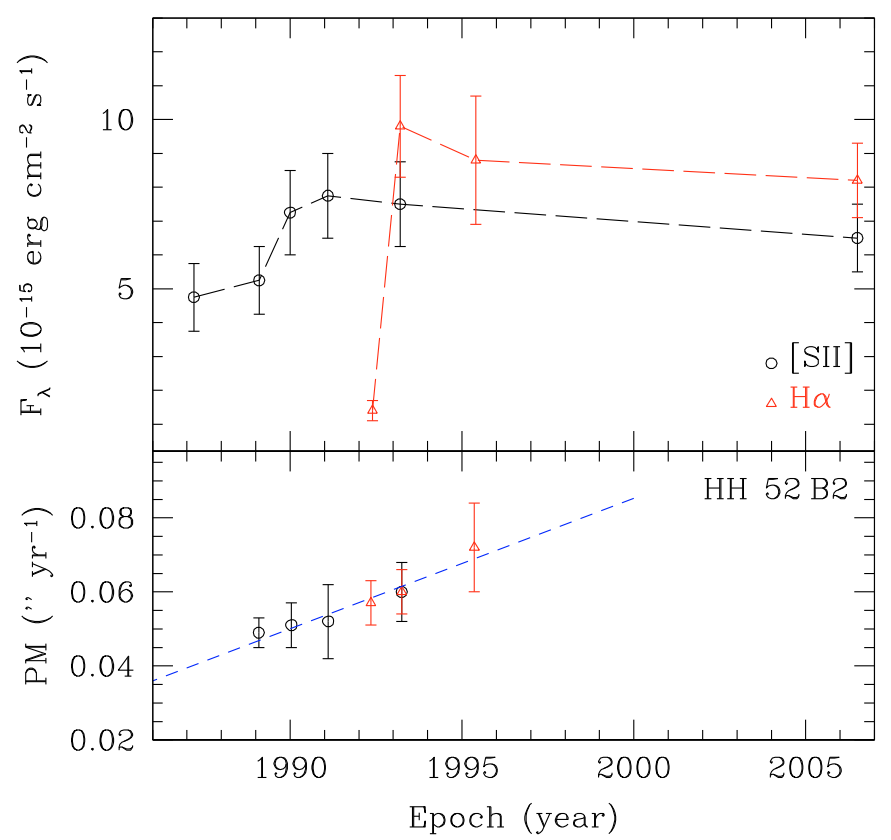

Fig. C.2. Variability in HH 52 B2. Bottom panel shows the PMs as a function of time in [S II] (circles)and $\mathrm{H} \alpha$ (triangles). The dashed line is the best fit to the data points. The slope of the fit gives the acceleration of the knot. In the top panel of the figure the measured fluxes (uncorrected for the extinction) in the two filters are reported.

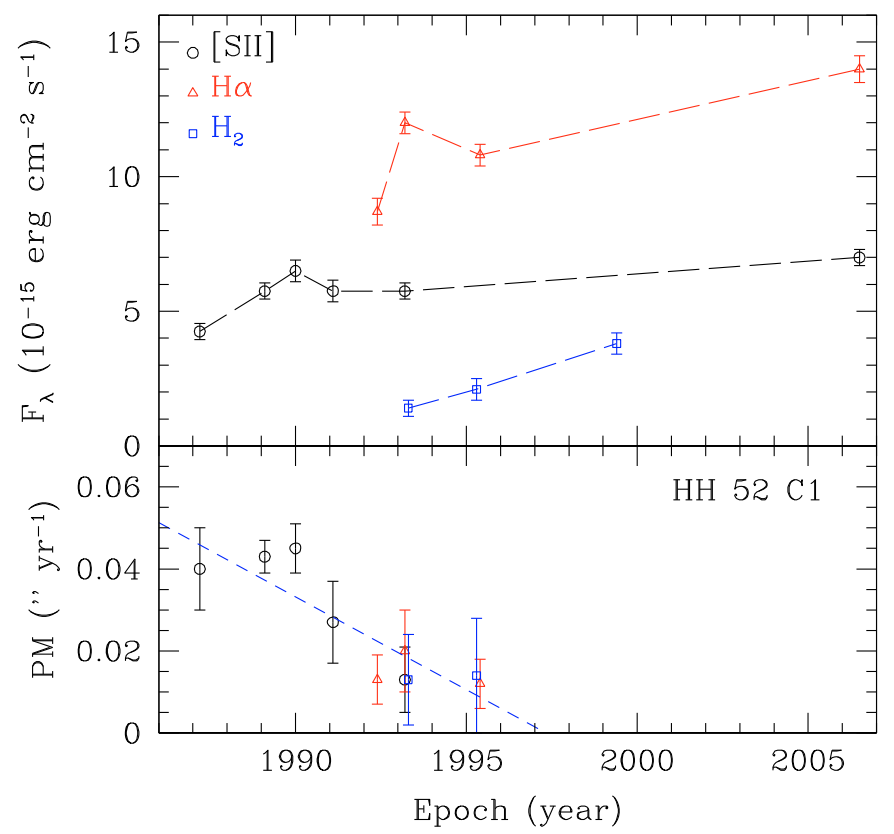

Fig. C.3. Variability in HH $52 \mathrm{C} 1$. Bottom panel shows the PMs as a function of time in [S II] (circles)and $\mathrm{H} \alpha$ (triangles). The dashed line is the best fit to the data points. The slope of the fit gives the acceleration of the knot. In the top panel of the figure the measured fluxes (uncorrected for the extinction) in the two filters are reported.

accelerated (see Figs. C.6, C.7), likely by the same mechanism of $\mathrm{HH} 54 \mathrm{G} / \mathrm{GO}$.

\section{C.2.4. $\mathrm{HH} 54 \mathrm{H}$}

Group $\mathrm{HH} 54 \mathrm{H}$ is a very active and fast variable region of the flow.

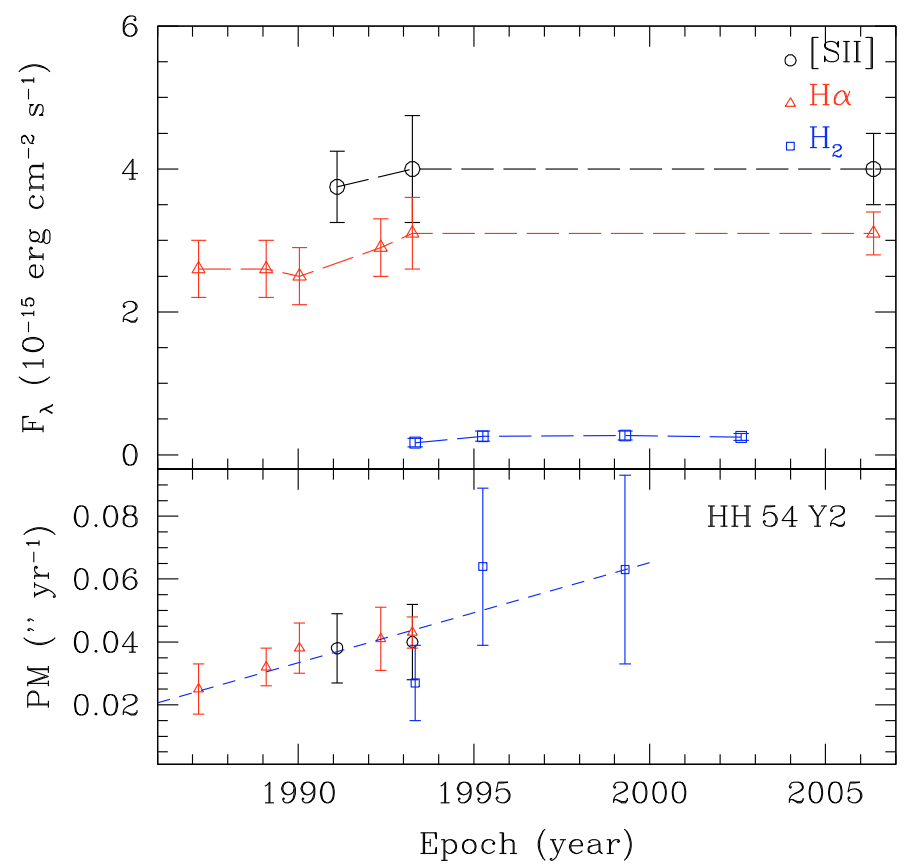

Fig. C.4. Variability in HH 54 Y2. Bottom panel shows the PMs as a function of time in [S II] (circles), $\mathrm{H} \alpha$ (triangles), and $\mathrm{H}_{2}$ (squares). The dashed line is the best fit to the data points. The slope of the fit gives the acceleration of the knot. In the top panel of the figure the measured fluxes (uncorrected for the extinction) in the three filters are reported.

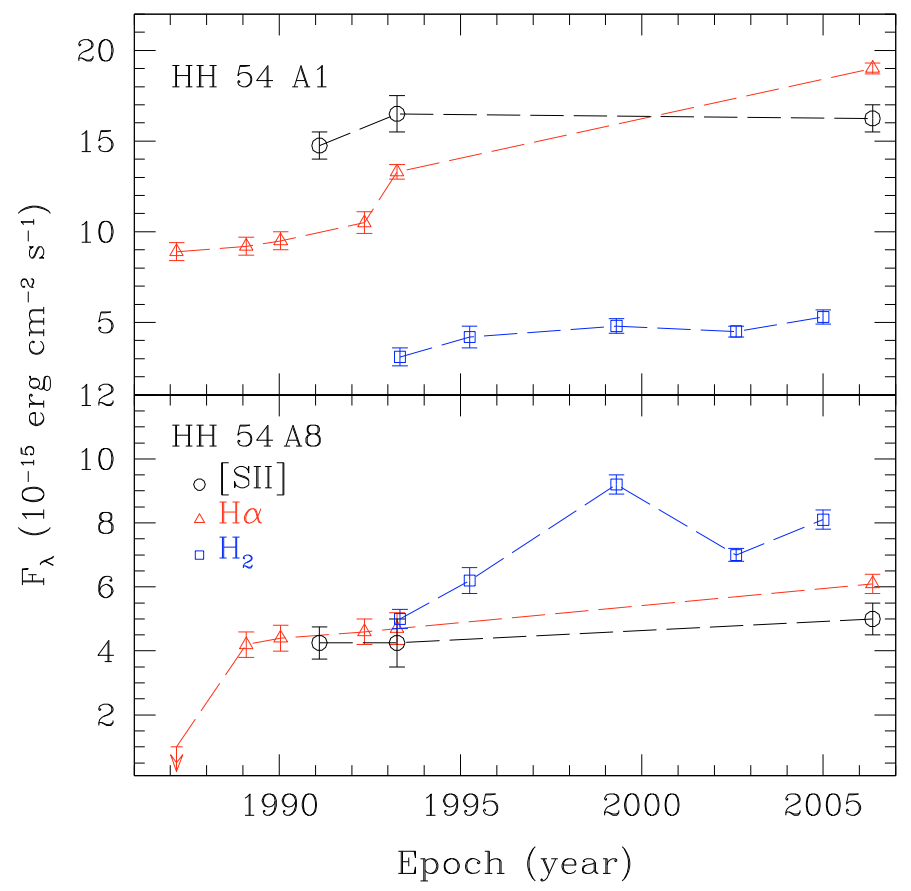

Fig. C.5. Measured fluxes (uncorrected for the extinction) in knots $\mathrm{HH} 54 \mathrm{~A} 1$ (bottom panel), and A8 (top panel) in [S II] (circles), H $\alpha$ (triangles), and $\mathrm{H}_{2}$ (squares) filters.

Between 1987 and $1993 \mathrm{H} 1$ has decreased the speed in the optical bands from 40 to $20 \mathrm{~km} \mathrm{~s}^{-1}$ with a PM deceleration of $0.02 \pm 0.01^{\prime \prime} \mathrm{yr}^{-2}$ in $\mathrm{H} \alpha$ (see Table C.1), and a value of $0.0025 \pm 0.0012^{\prime \prime} \mathrm{yr}^{-2}$, or $0.0063 \pm 0.0005^{\prime \prime} \mathrm{yr}^{-2}$ without considering 1993 data, combining all the optical PMs (see upper Fig. C.8, bottom panel). During this period the $\mathrm{H} \alpha$ and [S II] fluxes gradually increase from $4.9 \times 10^{-15} \mathrm{erg} \mathrm{s}^{-1} \mathrm{~cm}^{-2}$ 
A. Caratti o Garatti et al.: Time-variability in the Chamaeleontis II outflows, Online Material p 11

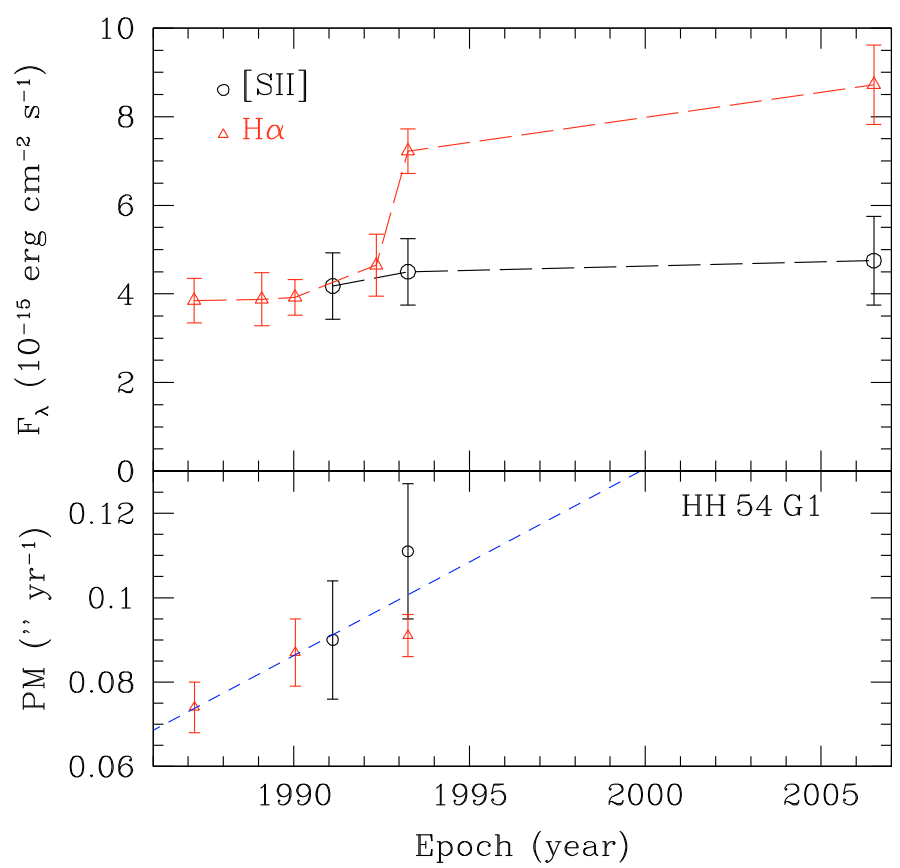

Fig. C.6. Variability in HH 54 G1. Bottom panel shows the PMs as a function of time in [S II] (circles) and $\mathrm{H} \alpha$ (triangles). The dashed line is the best fit to the data points. The slope of the fit gives the acceleration of the knot. In the top panel of the figure the measured fluxes (uncorrected for the extinction) in the two filters are reported.

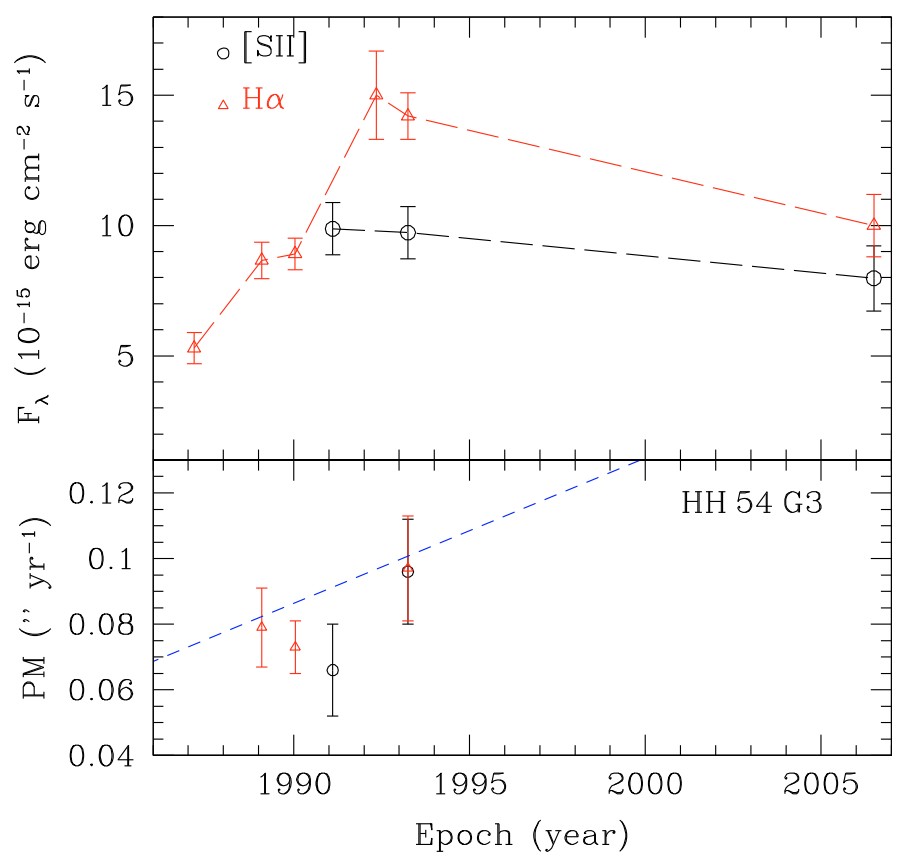

Fig. C.7. Variability in HH 54 G3. Bottom panel shows the PMs as a function of time in [S II] (circles) and $\mathrm{H} \alpha$ (triangles). The dashed line is the best fit to the data points. The slope of the fit gives the acceleration of the knot. In the top panel of the figure the measured fluxes (uncorrected for the extinction) in the two filters are reported.

to $7.2 \times 10^{-15} \mathrm{erg} \mathrm{s}^{-1} \mathrm{~cm}^{-2}$, and $1.8 \times 10^{-15} \mathrm{erg} \mathrm{s}^{-1} \mathrm{~cm}^{-2}$ to $2.8 \times 10^{-15} \mathrm{erg} \mathrm{s}^{-1} \mathrm{~cm}^{-2}$, respectively (top panel).

Knot H2 (see lower Fig. C.8, bottom panel) apparently drops in velocity between 1987 and $1989 \mathrm{H} \alpha$, changing the PM value from $\sim 0.08$ to $0.04^{\prime \prime} \mathrm{yr}^{-1}$ (decelerating of $0.022 \pm 0.002^{\prime \prime} \mathrm{yr}^{-2}$ in $\mathrm{H} \alpha$, see Table C.1). The knot has also a relevant flux variability
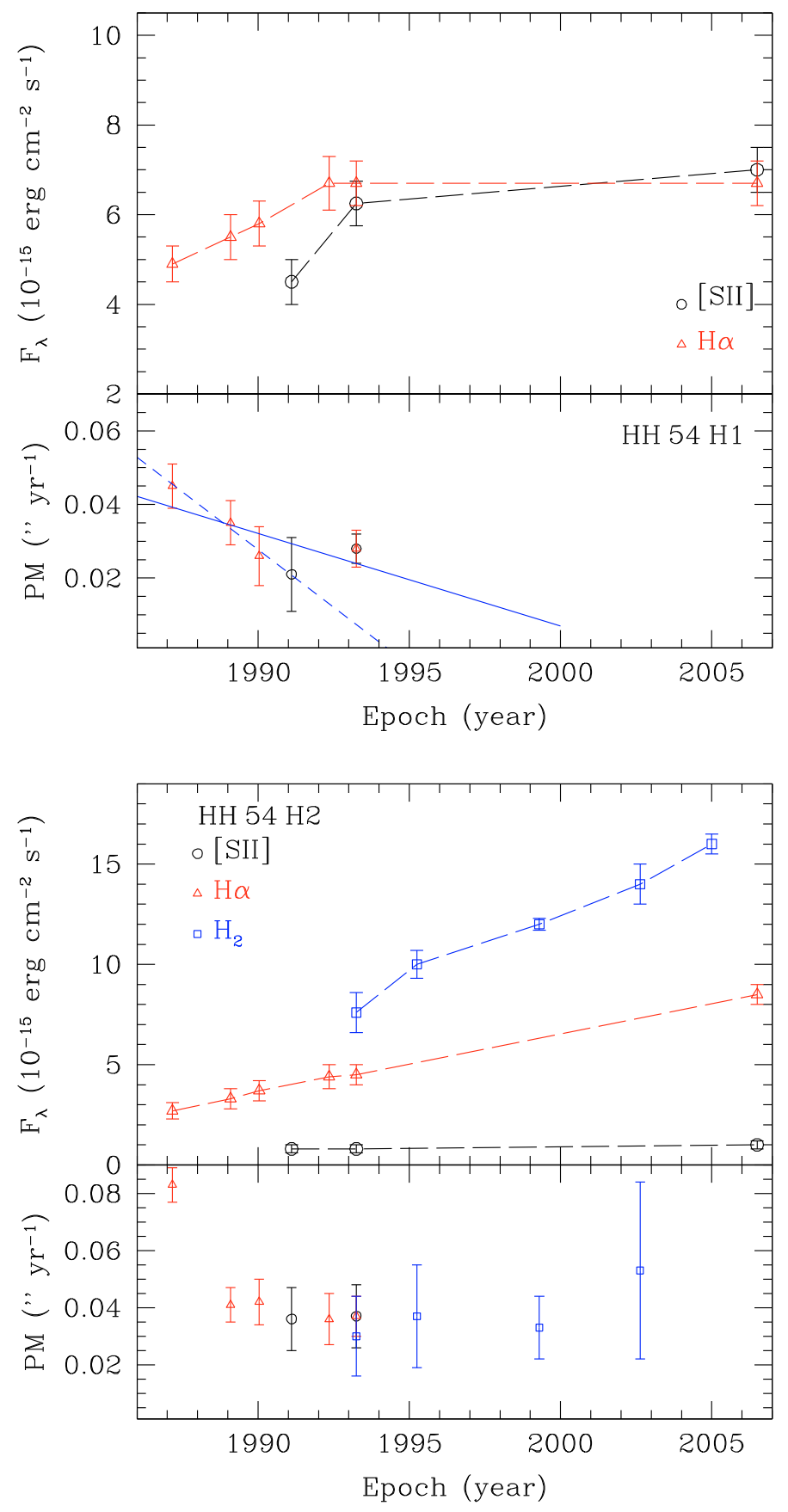

Fig. C.8. Variability in HH $54 \mathrm{H} 1$ upper Figure and $\mathrm{H} 2$ bottom Figure The bottom panels show the PMs as a function of time in both [S II] (circles) and $\mathrm{H} \alpha$ (triangles). The continuous and dashed lines in the top figure are the best fits of the data points with and without 1993 data points (see text), respectively. The slope of the fits gives the deceleration of the knot. In the top panel of the figures the measured fluxes (uncorrected for the extinction) in the two filters are reported.

at least in the $\mathrm{H} \alpha$ and $\mathrm{H}_{2}$ emissions, that have a constant increase of the flux from $2.7 \times 10^{-15} \mathrm{erg} \mathrm{s}^{-1} \mathrm{~cm}^{-2}$ (1987) to $8.5 \times 10^{-15} \mathrm{erg} \mathrm{s}^{-1} \mathrm{~cm}^{-2}(2006)$ in $\mathrm{H} \alpha$, and from $7.6 \times$ $10^{-15} \mathrm{erg} \mathrm{s}^{-1} \mathrm{~cm}^{-2}$ (1993) to $16 \times 10^{-15} \mathrm{erg} \mathrm{s}^{-1} \mathrm{~cm}^{-2}$ (2005) in $\mathrm{H}_{2}$. On the other hand the measured flux in the three $[\mathrm{S} \mathrm{II}] \mathrm{im}-$ ages is constant.

Finally H3 emission appears only in 2006 optical images, whereas in $\mathrm{H}_{2}$ it appears as a faint featureless emission and no significant motion is detectable. Again, the gap between the 
second to last and the last optical images makes it hard to derive the initial position of the condensation. We have tried to correlate it with a feature close to $\mathrm{H} 2$ in 1993 , but the interpretation is not certain.

\section{Appendix D: Radial velocity}

\section{D.1. HH52 flow}

Along the HH 52 flow, knots have radial velocities from about -70 to $-90 \mathrm{~km} \mathrm{~s}^{-1}$, with lower values detected at the end of the flow, in $\mathrm{HH} 54$, where the velocity decreases moving from $\mathrm{G}$ to $\mathrm{C}$, indicating that the flow has been decelerated. The lowest value is detected in $\mathrm{HH} 54 \mathrm{C} 2\left(-18 \pm 4 \mathrm{~km} \mathrm{~s}^{-1}\right)$ at the very end of the flow. C3 is following $\mathrm{C} 2$ at a higher velocity $(-45 \pm$ $4 \mathrm{~km} \mathrm{~s}^{-1}$ ) (see Table D.3). Along the jet, in knots G1-G3, we observe two distinct velocity components of -87 and $-21 \mathrm{~km} \mathrm{~s}^{-1}$. In the EMMI spectral image the emissions are well separated, delineating two distinct lines of similar intensities for each detected species (see Table D.3). At the base of the jet, where G0 and $\mathrm{G}$ are located, the fast component, extending a few arcseconds from the HH 52 streamer to G0, shows an abrupt deceleration (from -78 to $-18 \mathrm{~km} \mathrm{~s}^{-1}$ ) and then ahead, along the flow, a second slower velocity component is detected. Also in consideration of the proper motion analysis, where a portion of the flow appears to be deflected in this region, the two velocities could be ascribed to the two flow components, rather than a bow shock structure. Moreover, due to the profile of the observed emission lines, a hypothetical bow shock would lie close the plane of the sky (see e.g. Hartigan et al. 1987), but, as the inclination angle suggests (see also Sect. 4.6), this is not the case.

Two velocities components, not completely separated, are also detected in HH 52 knots located in front (D4) and behind $(\mathrm{A} 3-\mathrm{A} 4)$ the nucleus $\left(\mathrm{A} 1, v_{\text {rad }}=-79 \pm 6 \mathrm{~km} \mathrm{~s}^{-1}\right)$ (see Table D.1). Velocities in A3-A4 are slightly higher $(-98 \pm$ $5 \mathrm{~km} \mathrm{~s}^{-1}$ and $\left.-35 \pm 6 \mathrm{~km} \mathrm{~s}^{-1}\right)$, with respect to D4 $(-85 \pm$ $3 \mathrm{~km} \mathrm{~s}^{-1}$ and $-30 \pm 3 \mathrm{~km} \mathrm{~s}^{-1}$ ). Line fluxes of the low velocity component are considerably fainter than the other component. The velocity profiles of the $\mathrm{H} \alpha$ line in both structures closely match the theoretical profile of a bow shock with an inclination angle of $\sim 60^{\circ}$ with respect to the plane of the sky (see Hartigan et al. 1987) and a shock velocity of $70-80 \mathrm{~km} \mathrm{~s}^{-1}$ (observed from the full width zero intensity - FWZI, measured on the spectrum where the flux reaches a $2 \sigma$ background noise level, see e.g. Davis et al. 2001).

\section{D.2. HH53 flow}

The highest $v_{\text {rad }}$ values are observed in the three knots of HH 53 (see Table D.2) outflow, A $\left(-110 \pm 10 \mathrm{~km} \mathrm{~s}^{-1}\right)$, B $(-113 \pm$ $\left.9 \mathrm{~km} \mathrm{~s}^{-1}\right)$, and $\mathrm{C}\left(-96 \pm 14 \mathrm{~km} \mathrm{~s}^{-1}\right)$, derived averaging the radial velocities of the different species from $\mathrm{B} \& \mathrm{C}$ slit 1 and 2 . In the EMMI spectrum (Table D.2) it is not possible to spatially disentangle the emission coming from knots $\mathrm{C}$ and $\mathrm{C} 1$. Here, however, we measure two different velocities of $-104 \pm 7 \mathrm{~km} \mathrm{~s}^{-1}$ and $-55 \pm 18 \mathrm{~km} \mathrm{~s}^{-1}$. Considering that the two knots are from two separate flows, the higher velocity should be ascribed to knot $\mathrm{C}$ and the lower to $\mathrm{C} 1$.

\section{D.3. HH54 flow}

Along the portion of the HH54 streamer encompassed by our B\&C slits, we measure an increase in the velocity moving towards the main body of HH54 (see also Table D.3).
Table D.1. Radial velocities and line intensities for different optical lines of individual knots in HH 52 from EMMI and B\&C spectra. Radial velocities are corrected for the cloud speed with respect to the LSR $\left(v_{\mathrm{LSR}}=2 \mathrm{~km} \mathrm{~s}^{-1}\right.$, Knee 1992). When detected, two velocity components are reported.

\begin{tabular}{|c|c|c|c|c|}
\hline \multirow[b]{2}{*}{ Knot } & \multicolumn{4}{|c|}{ " } \\
\hline & $\begin{array}{l}\text { Line } \\
\text { Id. }\end{array}$ & $\begin{array}{c}\lambda \\
(\AA)\end{array}$ & $\begin{array}{c}v_{\mathrm{rad}} \\
\left(\mathrm{km} \mathrm{s}^{-1}\right)\end{array}$ & $\begin{array}{c}F \pm \Delta F \\
\left(10^{-15} \mathrm{erg} \mathrm{s}^{-1} \mathrm{~cm}^{-2}\right)\end{array}$ \\
\hline \multirow{8}{*}{ A1 } & & & EMMI & \\
\hline & {$[\mathrm{O} \mathrm{I}]$} & 6300.3 & $-78 \pm 4$ & $8.1 \pm 0.1$ \\
\hline & {$[\mathrm{OI}]$} & 6363.8 & $-77 \pm 4$ & $2.9 \pm 0.1$ \\
\hline & {$[\mathrm{N}$ II $]$} & 6548.1 & $-78 \pm 4$ & $2.2 \pm 0.1$ \\
\hline & $\mathrm{H} \alpha$ & 6562.8 & $-70 \pm 4$ & $16.7 \pm 0.2$ \\
\hline & {$[\mathrm{N}$ II $]$} & 6583.4 & $-79 \pm 4$ & $5.8 \pm 0.1$ \\
\hline & {$[\mathrm{S}$ II $]$} & 6716.4 & $-87 \pm 4$ & $12.0 \pm 0.1$ \\
\hline & [S II] & 6730.8 & $-86 \pm 4$ & $12.4 \pm 0.1$ \\
\hline \multirow[t]{7}{*}{$\mathrm{A} 3-\mathrm{A} 4$} & {$[\mathrm{OI}]$} & 6300.3 & $-89 \pm 4 ;-43 \pm 4$ & $1.5 \pm 0.1 ; 1.4 \pm 0.1$ \\
\hline & {$[\mathrm{OI}]$} & 6363.8 & - & $1.0 \pm 0.1$ \\
\hline & {$[\mathrm{N} \mathrm{II}]$} & 6548.1 & $-96 \pm 4 ;-27 \pm 4$ & $1.5 \pm 0.1 ; 0.5 \pm 0.1$ \\
\hline & $\mathrm{H} \alpha$ & 6562.8 & $-101 \pm 4 ;-40 \pm 4$ & $2.3 \pm 0.2 ; 1.9 \pm 0.2$ \\
\hline & {$[\mathrm{N}$ II $]$} & 6583.4 & - & $0.3 \pm 0.1$ \\
\hline & {$[\mathrm{S}$ II $]$} & 6716.4 & $-100 \pm 4 ;-34 \pm 4$ & $2.1 \pm 0.1 ; 1.3 \pm 0.1$ \\
\hline & [S II $]$ & 6730.8 & $-102 \pm 4 ;-31 \pm 4$ & $1.8 \pm 0.2 ; 1.0 \pm 0.1$ \\
\hline \multirow[t]{6}{*}{ D4 } & {$[\mathrm{OI}]$} & 6300.3 & $-84 \pm 4 ;-30 \pm 4$ & $0.7 \pm 0.1 ; 0.3 \pm 0.1$ \\
\hline & [N II] & 6548.1 & $-84 \pm 4 ;-33 \pm 4$ & $0.8 \pm 0.1 ; 0.4 \pm 0.1$ \\
\hline & $\mathrm{H} \alpha$ & 6562.8 & $-73 \pm 4^{a}$ & $2.4 \pm 0.2$ \\
\hline & {$[\mathrm{N}$ II $]$} & 6583.4 & - & $0.5 \pm 0.2^{b}$ \\
\hline & [S II $]$ & 6716.4 & $-87 \pm 4 ;-27 \pm 4$ & $1.4 \pm 0.1 ; 0.5 \pm 0.1$ \\
\hline & [S II] & 6730.8 & $-87 \pm 4 ;-29 \pm 4$ & $1.1 \pm 0.1 ; 0.5 \pm 0.1$ \\
\hline \multirow{8}{*}{$\mathrm{A}^{c}$} & & & $\mathrm{~B} \& \mathrm{C}$ & \\
\hline & {$[\mathrm{O} I]$} & 6300.3 & $-102 \pm 20$ & $40.0 \pm 0.2$ \\
\hline & {$[\mathrm{O} \mathrm{I}]$} & 6363.8 & $-103 \pm 20$ & $14.3 \pm 0.2$ \\
\hline & {$[\mathrm{N}$ II $]$} & 6548.1 & $-94 \pm 20$ & $10.3 \pm 0.2$ \\
\hline & $\mathrm{H} \alpha$ & 6562.8 & $-101 \pm 20$ & $95.6 \pm 0.3$ \\
\hline & {$[\mathrm{N}$ II $]$} & 6583.4 & $-89 \pm 20$ & $31.9 \pm 0.2$ \\
\hline & [S II $]$ & 6716.4 & $-81 \pm 20$ & $62.8 \pm 0.3$ \\
\hline & {$[\mathrm{S}$ II $]$} & 6730.8 & $-78 \pm 20$ & $58.7 \pm 0.3$ \\
\hline \multirow[t]{7}{*}{$\mathrm{B}^{d}$} & {$[\mathrm{OI}]$} & 6300.3 & $-77 \pm 20$ & $5.3 \pm 0.4$ \\
\hline & {$[\mathrm{OI}]$} & 6363.8 & $-83 \pm 20$ & $2.2 \pm 0.3$ \\
\hline & {$[\mathrm{N}$ II $]$} & 6548.1 & $-85 \pm 20$ & $2.6 \pm 0.2$ \\
\hline & $\mathrm{H} \alpha$ & 6562.8 & $-85 \pm 20$ & $17.2 \pm 0.2$ \\
\hline & [N II] & 6583.4 & $-85 \pm 20$ & $8.1 \pm 0.2$ \\
\hline & [S II] & 6716.4 & $-63 \pm 20$ & $6.5 \pm 0.2$ \\
\hline & [S II $]$ & 6730.8 & $-67 \pm 20$ & $6.8 \pm 0.2$ \\
\hline \multirow[t]{7}{*}{ D2-D3 } & {$[\mathrm{OI}]$} & 6300.3 & $-112 \pm 20$ & $7.7 \pm 0.2$ \\
\hline & [O I] & 6363.8 & $-132 \pm 20$ & $3.5 \pm 0.2$ \\
\hline & [N II] & 6548.1 & $-112 \pm 20$ & $1.4 \pm 0.2$ \\
\hline & $\mathrm{H} \alpha$ & 6562.8 & $-94 \pm 20$ & $25.3 \pm 0.3$ \\
\hline & {$\left[\mathrm{N}_{\mathrm{II}}\right]$} & 6583.4 & $-103 \pm 20$ & $5.3 \pm 0.3$ \\
\hline & [S II $]$ & 6716.4 & $-103 \pm 20$ & $13.1 \pm 0.3$ \\
\hline & [S II $]$ & 6730.8 & $-99 \pm 20$ & $10.6 \pm 0.2$ \\
\hline
\end{tabular}

Notes: ${ }^{a}$ not possible to deblend; ${ }^{b} \mathrm{~S} / \mathrm{N}$ between 2 and $3 ;{ }^{c}$ blend of knots $\mathrm{A} 1, \mathrm{~A} 3, \mathrm{~A} 4, \mathrm{~A} 6, \mathrm{~A} 7 ;^{d}$ blend of knots B5, B8, B9.

The values rise from about $-50 \mathrm{~km} \mathrm{~s}^{-1}$, roughly at the base of the streamer $(\mathrm{X} 1 \mathrm{~A}-\mathrm{X} 3)$, up to $-100 \mathrm{~km} \mathrm{~s}^{-1}$, close to the middle (X4A-X4B). Such a behaviour was also observed by Graham \& Hartigan (1988), but the value measured in the middle (considering also the errors) is lower than ours of $\sim 10-20 \mathrm{~km} \mathrm{~s}^{-1}$. Such a difference in their values could be due to a not perfect positioning of the slit, due to the sinuous geometry of the HH 54 streamer. 
Table D.2. Radial velocities and line intensities for different optical lines of individual knots in HH 53 from EMMI and B\&C spectra. Radial velocities are corrected for the cloud speed with respect to the LSR $\left(v_{\text {LSR }}=2 \mathrm{~km} \mathrm{~s}^{-1}\right.$, Knee 1992). When detected, two velocity components are reported.

\begin{tabular}{|c|c|c|c|c|}
\hline \multicolumn{5}{|c|}{$\overline{\mathrm{HH} 53}$} \\
\hline Knot & $\begin{array}{l}\text { Line } \\
\text { Id. }\end{array}$ & $\begin{array}{c}\lambda \\
(\AA)\end{array}$ & $\begin{array}{c}v_{\mathrm{rad}} \\
\left(\mathrm{km} \mathrm{s}^{-1}\right)\end{array}$ & $\begin{array}{c}F \pm \Delta F \\
\left(10^{-15} \mathrm{erg} \mathrm{s}^{-1} \mathrm{~cm}^{-2}\right)\end{array}$ \\
\hline \multirow{7}{*}{$\mathrm{C}-\mathrm{C} 1$} & & & EMMI & \\
\hline & {$[\mathrm{O} \mathrm{I}]$} & 6300.3 & $-101 \pm 4 ;-32 \pm 4$ & $1.9 \pm 0.1 ; 0.9 \pm 0.2$ \\
\hline & {$[\mathrm{OI}]$} & 6363.8 & - & $1.0 \pm 0.4$ \\
\hline & $\mathrm{H} \alpha$ & 6562.8 & $-97 \pm 4 ;-47 \pm 4$ & $9.8 \pm 0.1 ; 2.4 \pm 0.2$ \\
\hline & {$[\mathrm{N}$ II $]$} & 6583.4 & $-100 \pm 4 ;-51 \pm 4$ & $1.5 \pm 0.1 ; 0.4 \pm 0.2$ \\
\hline & {$[\mathrm{S}$ II $]$} & 6716.4 & $-111 \pm 4 ;-72 \pm 4$ & $2.2 \pm 0.1 ; 0.7 \pm 0.1$ \\
\hline & {$[\mathrm{S} \mathrm{II}]$} & 6730.8 & $-112 \pm 4 ;-74 \pm 4$ & $2.0 \pm 0.1 ; 0.7 \pm 0.1$ \\
\hline \multirow{3}{*}{ E1 } & $\mathrm{H} \alpha$ & 6562.8 & $-65 \pm 4$ & $0.9 \pm 0.1$ \\
\hline & {$[\mathrm{S} \mathrm{II}]$} & 6716.4 & $-74 \pm 4$ & $0.7 \pm 0.1$ \\
\hline & [S II] & 6730.8 & $-76 \pm 4$ & $0.7 \pm 0.1$ \\
\hline \multirow{6}{*}{ A } & & & B\&C slit 1 & \\
\hline & {$[\mathrm{O} \mathrm{I}]$} & 6300.3 & $-140 \pm 30$ & $4.2 \pm 0.8$ \\
\hline & $\mathrm{H} \alpha$ & 6562.8 & $-114 \pm 30$ & $25.0 \pm 0.6$ \\
\hline & {$[\mathrm{N}$ III $]$} & 6583.4 & $-104 \pm 30$ & $5.3 \pm 0.8$ \\
\hline & {$[\mathrm{S} \mathrm{II}]$} & 6716.4 & $-117 \pm 30$ & $6.6 \pm 0.4$ \\
\hline & {$[\mathrm{S} \mathrm{II}]$} & 6730.8 & $-128 \pm 30$ & $6.4 \pm 0.5$ \\
\hline \multirow[t]{6}{*}{ B } & [O I $]$ & 6300.3 & $-130 \pm 30$ & $6.3 \pm 0.6$ \\
\hline & {$[\mathrm{N}$ II $]$} & 6548.1 & $-141 \pm 30$ & $2.5 \pm 0.6$ \\
\hline & $\mathrm{H} \alpha$ & 6562.8 & $-124 \pm 30$ & $34.3 \pm 0.6$ \\
\hline & {$[\mathrm{N}$ III $]$} & 6583.4 & $-103 \pm 30$ & $3.6 \pm 0.5$ \\
\hline & {$[\mathrm{S}$ II $]$} & 6716.4 & $-139 \pm 30$ & $10.5 \pm 0.5$ \\
\hline & {$[\mathrm{S} \mathrm{II}]$} & 6730.8 & $-127 \pm 30$ & $9.0 \pm 0.6$ \\
\hline \multirow[t]{3}{*}{$\mathrm{C}$} & $\mathrm{H} \alpha$ & 6562.8 & $-102 \pm 30$ & $8.8 \pm 0.6$ \\
\hline & {$[\mathrm{N}$ II] } & 6583.4 & $-103 \pm 30$ & $2.7 \pm 0.5$ \\
\hline & {$[\mathrm{S} \mathrm{II}]$} & 6716.4 & $-101 \pm 30$ & $2.1 \pm 0.6$ \\
\hline \multirow{8}{*}{ A } & & & B\&C slit 2 & \\
\hline & {$[\mathrm{O} \mathrm{I}]$} & 6300.3 & $-96 \pm 20$ & $6.7 \pm 0.1$ \\
\hline & {$[\mathrm{OI}]$} & 6363.8 & $-112 \pm 20$ & $2.2 \pm 0.1$ \\
\hline & {$[\mathrm{N}$ II] } & 6548.1 & $-76 \pm 20$ & $1.6 \pm 0.1$ \\
\hline & $\mathrm{H} \alpha$ & 6562.8 & $-106 \pm 20$ & $26.3 \pm 0.1$ \\
\hline & {$[\mathrm{N}$ III $]$} & 6583.4 & $-82 \pm 20$ & $5.1 \pm 0.1$ \\
\hline & {$[\mathrm{S} \mathrm{II}]$} & 6716.4 & $-115 \pm 20$ & $7.2 \pm 0.1$ \\
\hline & {$[\mathrm{S} \mathrm{II}]$} & 6730.8 & $-111 \pm 20$ & $7.8 \pm 0.1$ \\
\hline \multirow[t]{7}{*}{ B } & {$[\mathrm{O} \mathrm{I}]$} & 6300.3 & $-97 \pm 20$ & $9.7 \pm 0.1$ \\
\hline & {$[\mathrm{O} \mathrm{I}]$} & 6363.8 & $-96 \pm 20$ & $2.9 \pm 0.1$ \\
\hline & {$[\mathrm{N}$ II $]$} & 6548.1 & $-94 \pm 20$ & $2.1 \pm 0.1$ \\
\hline & $\mathrm{H} \alpha$ & 6562.8 & $-98 \pm 20$ & $33.3 \pm 0.1$ \\
\hline & {$[\mathrm{N}$ II $]$} & 6583.4 & $-94 \pm 20$ & $6.9 \pm 0.1$ \\
\hline & {$[\mathrm{S} \mathrm{II}]$} & 6716.4 & $-106 \pm 20$ & $11.5 \pm 0.1$ \\
\hline & {$[\mathrm{S}$ II] } & 6730.8 & $-112 \pm 20$ & $11.3 \pm 0.1$ \\
\hline \multirow[t]{7}{*}{$\mathrm{C}$} & {$[\mathrm{O} \mathrm{I}]$} & 6300.3 & $-109 \pm 20$ & $3.1 \pm 0.1$ \\
\hline & [O I $]$ & 6363.8 & $-125 \pm 20$ & $1.1 \pm 0.1$ \\
\hline & {$[\mathrm{N}$ II $]$} & 6548.1 & $-120 \pm 20$ & $0.6 \pm 0.1$ \\
\hline & $\mathrm{H} \alpha$ & 6562.8 & $-122 \pm 20$ & $14.2 \pm 0.1$ \\
\hline & {$[\mathrm{N}$ II] } & 6583.4 & $-120 \pm 20$ & $2.0 \pm 0.1$ \\
\hline & {$[\mathrm{S} \mathrm{II}]$} & 6716.4 & $-116 \pm 20$ & $4.5 \pm 0.1$ \\
\hline & {$[\mathrm{S}$ II $]$} & 6730.8 & $-111 \pm 20$ & $3.9 \pm 0.1$ \\
\hline \multirow[t]{7}{*}{$\mathrm{F} 2$} & {$[\mathrm{O} \mathrm{I}]$} & 6300.3 & $-87 \pm 20$ & $1.5 \pm 0.1$ \\
\hline & [O I] & 6363.8 & - & $0.8 \pm 0.4$ \\
\hline & {$[\mathrm{N}$ II] } & 6548.1 & - & $1.0 \pm 0.2$ \\
\hline & $\mathrm{H} \alpha$ & 6562.8 & $-97 \pm 20$ & $7.3 \pm 0.2$ \\
\hline & {$[\mathrm{N} \mathrm{III}]$} & 6583.4 & $-85 \pm 20$ & $1.7 \pm 0.2$ \\
\hline & {$[\mathrm{S}$ II] } & 6716.4 & $-81 \pm 20$ & $2.1 \pm 0.2$ \\
\hline & {$[\mathrm{S}$ II] } & 6730.8 & $-95 \pm 20$ & $1.7 \pm 0.2$ \\
\hline
\end{tabular}

Notes: ${ }^{a}$ not possible to deblend; ${ }^{b} \mathrm{~S} / \mathrm{N}$ between 2 and 3.

\section{Appendix E: Inclination and spatial velocity}

In HH 52 bow shock we obtain an average inclination with respect to the sky plane of $58^{\circ} \pm 3^{\circ}$, excluding D2-D3
Table D.3. Radial velocities and line intensities for different optical lines of individual knots in HH 54 from EMMI and B\&C spectra. Radial velocities are corrected for the cloud speed with respect to the LSR $\left(v_{\text {LSR }}=2 \mathrm{~km} \mathrm{~s}^{-1}\right.$, Knee 1992). When detected, two velocity components are reported.

\begin{tabular}{|c|c|c|c|c|}
\hline \multirow[b]{2}{*}{ Knot } & \multicolumn{4}{|c|}{$\overline{\mathrm{HH}} 54$} \\
\hline & $\begin{array}{l}\text { Line } \\
\text { Id. }\end{array}$ & $\begin{array}{c}\lambda \\
(\AA)\end{array}$ & $\begin{array}{c}v_{\mathrm{rad}} \\
\left(\mathrm{km} \mathrm{s}^{-1}\right)\end{array}$ & $\begin{array}{c}F \pm \Delta F \\
\left(10^{-15} \mathrm{erg} \mathrm{s}^{-1} \mathrm{~cm}^{-2}\right)\end{array}$ \\
\hline \multirow{3}{*}{$\mathrm{C} 2$} & & & EMMI & \\
\hline & {$[\mathrm{OI}]$} & 6300.3 & $-40 \pm 4$ & $6.4 \pm 0.3$ \\
\hline & {$[\mathrm{OI}]$} & 6363.8 & $-43 \pm 4$ & $2.8 \pm 0.3$ \\
\hline \multirow{11}{*}{$\mathrm{C} 3$} & {$[\mathrm{~N}$ II $]$} & 6548.1 & $-47 \pm 4$ & $2.2 \pm 0.2$ \\
\hline & $\mathrm{H} \alpha$ & 6562.8 & $-52 \pm 4$ & $13.9 \pm 0.3$ \\
\hline & {$[\mathrm{N}$ II $]$} & 6583.4 & $-44 \pm 4$ & $6.5 \pm 0.2$ \\
\hline & {$[\mathrm{S} \mathrm{II}]$} & 6716.4 & $-46 \pm 4$ & $8.9 \pm 0.2$ \\
\hline & {$[\mathrm{S}$ II $]$} & 6730.8 & $-46 \pm 4$ & $8.9 \pm 0.2$ \\
\hline & {$[\mathrm{OI}]$} & 6300.3 & $-12 \pm 4$ & $1.5 \pm 0.1$ \\
\hline & {$[\mathrm{OI}]$} & 6363.8 & $-17 \pm 4$ & $0.7 \pm 0.2$ \\
\hline & {$[\mathrm{N}$ II $]$} & 6548.1 & $-16 \pm 4$ & $0.4 \pm 0.1$ \\
\hline & $\mathrm{H} \alpha$ & 6562.8 & $-19 \pm 4$ & $2.8 \pm 0.1$ \\
\hline & {$[\mathrm{N}$ II $]$} & 6583.4 & $-20 \pm 4$ & $1.6 \pm 0.1$ \\
\hline & {$[\mathrm{S}$ II] } & 6716.4 & $-17 \pm 4$ & $1.7 \pm 0.1$ \\
\hline \multirow{7}{*}{ G-G0 } & {$[\mathrm{S} \mathrm{II}]$} & 6730.8 & $-22 \pm 4$ & $1.8 \pm 0.1$ \\
\hline & {$[\mathrm{OI}]$} & 6300.3 & $-71 \pm 4 ;-24 \pm 4$ & $0.4 \pm 0.1 ; 0.6 \pm 0.1$ \\
\hline & {$[\mathrm{N}$ II $]$} & 6548.1 & - & $0.5 \pm 0.2 ;-$ \\
\hline & $\mathrm{H} \alpha$ & 6562.8 & $-80 \pm 4 ;-15 \pm 4$ & $3.0 \pm 0.1 ; 0.5 \pm 0.1$ \\
\hline & {$[\mathrm{N}$ II $]$} & 6583.4 & $-73 \pm 4 ;-15 \pm 4$ & $0.8 \pm 0.1 ; 0.6 \pm 0.1$ \\
\hline & {$[\mathrm{S} \mathrm{II}]$} & 6716.4 & $-78 \pm 4 ;-17 \pm 4$ & $1.0 \pm 0.2 ; 0.6 \pm 0.2$ \\
\hline & [S II] & 6730.8 & $-79 \pm 4 ;-21 \pm 4$ & $1.0 \pm 0.2 ; 0.5 \pm 0.2$ \\
\hline \multirow[t]{7}{*}{$\mathrm{G} 1-\mathrm{G}^{a}{ }^{a}$} & {$[\mathrm{OI}]$} & 6300.3 & $-86 \pm 4 ;-18 \pm 4$ & $1.9 \pm 0.1 ; 2.5 \pm 0.1$ \\
\hline & {$[\mathrm{OI}]$} & 6363.8 & - & $0.6 \pm 0.2 ; 0.8 \pm 0.2$ \\
\hline & {$[\mathrm{N}$ II $]$} & 6548.1 & - & $0.6 \pm 0.2 ; 1.0 \pm 0.3$ \\
\hline & $\mathrm{H} \alpha$ & 6562.8 & $-82 \pm 4 ;-24 \pm 4$ & $5.6 \pm 0.3 ; 5.0 \pm 0.3$ \\
\hline & {$[\mathrm{N}$ II $]$} & 6583.4 & $-86 \pm 4 ;-20 \pm 4$ & $1.2 \pm 0.2 ; 2.1 \pm 0.2$ \\
\hline & {$[\mathrm{S}$ II] } & 6716.4 & $-89 \pm 4 ;-20 \pm 4$ & $2.6 \pm 0.2 ; 2.3 \pm 0.2$ \\
\hline & [S II] & 6730.8 & $-91 \pm 4 ;-22 \pm 4$ & $1.6 \pm 0.2 ; 2.0 \pm 0.1$ \\
\hline \multirow{5}{*}{$\mathrm{X} 1 \mathrm{D}-\mathrm{X} 4 \mathrm{C}$} & & & B\&C slit 3 & \\
\hline & {$[\mathrm{OI}]$} & 6300.3 & $-34 \pm 30$ & $6.5 \pm 1.3$ \\
\hline & $\mathrm{H} \alpha$ & 6562.8 & $-70 \pm 30$ & $40.5 \pm 2.8$ \\
\hline & {$[\mathrm{N}$ II $]$} & 6583.4 & $-58 \pm 30$ & $5.4 \pm 2.0$ \\
\hline & [S II] & 6716.4 & $-61 \pm 30$ & $6.6 \pm 0.6$ \\
\hline \multirow{4}{*}{$\begin{array}{c}\mathrm{X} 1 \mathrm{~A} \\
\mathrm{X} 3\end{array}$} & {$[\mathrm{~S} \mathrm{II}]$} & 6730.8 & $-67 \pm 30$ & $6.3 \pm 2.5$ \\
\hline & $\mathrm{H} \alpha$ & 6562.8 & $-70 \pm 20$ & $4.5 \pm 0.1$ \\
\hline & [O I] & 6300.3 & $-40 \pm 20$ & $1.6 \pm 0.1$ \\
\hline & $\mathrm{H} \alpha$ & 6562.8 & $-42 \pm 20$ & $9.9 \pm 0.1$ \\
\hline \multirow{7}{*}{$\mathrm{X} 4 \mathrm{~A}-\mathrm{X} 4 \mathrm{~B}$} & {$[\mathrm{~N}$ II $]$} & 6583.4 & $-65 \pm 20$ & $1.1 \pm 0.1$ \\
\hline & [S II] & 6716.4 & $-53 \pm 20$ & $2.0 \pm 0.2$ \\
\hline & {$[\mathrm{S}$ II] } & 6730.8 & $-34 \pm 20$ & $1.3 \pm 0.2$ \\
\hline & $\mathrm{H} \alpha$ & 6562.8 & $-115 \pm 20$ & $11.8 \pm 0.2$ \\
\hline & {$[\mathrm{N}$ II $]$} & 6583.4 & $-110 \pm 20$ & $0.8 \pm 0.2$ \\
\hline & [S II] & 6716.4 & $-92 \pm 20$ & $1.8 \pm 0.2$ \\
\hline & {$[\mathrm{S}$ II $]$} & 6730.8 & $-82 \pm 20$ & $1.9 \pm 0.2$ \\
\hline
\end{tabular}

Notes: ${ }^{a}$ Blend of knots G1-G3.

measurement, that shows a value of $67^{\circ} \pm 4^{\circ}$. HH $53 \mathrm{E} 1$ and $\mathrm{F} 2$, as already deduced from the PM analysis, are part of the same outflow with inclinations of $57^{\circ} \pm 3^{\circ}$ and $63^{\circ} \pm 4^{\circ}$, respectively. The association of groups $\mathrm{HH} 54 \mathrm{G}$ and $\mathrm{C}$ to the HH52 outflow is also confirmed. HH $54 \mathrm{G0}$ has an inclination of $55^{\circ} \pm$ $3^{\circ}$, at the end of the streamer. Moreover, if we assume that along HH 54 G1-G3 the high and low radial velocities are associated with the high and low tangential velocities observed along the jet, we obtain inclinations ranging between $50^{\circ} \pm 4^{\circ}$ and $56^{\circ} \pm$ $5^{\circ}$, respectively. Values in HH $54 \mathrm{C} 2$ and $\mathrm{C} 3$ are $61^{\circ} \pm 3^{\circ}$ and $52^{\circ} \pm 8^{\circ}$, respectively. Spatial velocity along the flow are around 
Table D.4. [Fe II] radial velocities of the individual knots of HH 54 obtained from ISAAC high resolution spectroscopy, corrected for the cloud speed with respect to the LSR $\left(v_{\mathrm{LSR}}=2 \mathrm{~km} \mathrm{~s}^{-1}\right.$, Knee 1992). When detected, two velocities components are reported.

\begin{tabular}{cc}
\hline \hline \multicolumn{2}{c}{ HH 54 - ISAAC slit 1 } \\
knot & $v_{\mathrm{rad}}\left(\mathrm{km} \mathrm{s}^{-1}\right)$ \\
\hline A1 & $-52 \pm 3 ;-105 \pm 3$ \\
A3 & $-34 \pm 3$ \\
B & $-14 \pm 3 ;-109 \pm 3$ \\
B3 & $-49 \pm 3 ;-105 \pm 3$ \\
J-J1 & $-9 \pm 3 ;-91 \pm 3$ \\
H2 & $-36 \pm 3 ;-90 \pm 3$ \\
H3 & $-20 \pm 3 ;-45 \pm 3$ \\
Z & $-100 \pm 3$ \\
\hline HH $54-$ ISAAC slit 2 \\
knot & $v_{\text {rad }}\left(\mathrm{km} \mathrm{s}^{-1}\right)$ \\
\hline C1 & $-5 \pm 3 ;-90 \pm 3$ \\
C3 & $-9 \pm 3 ;-85 \pm 3$ \\
E & $-52 \pm 3$ \\
J-J1 & $-18 \pm 3 ;-62 \pm 3$ \\
H3 & $-40 \pm 3$ \\
K & $-62 \pm 3$ \\
K1 & $-45 \pm 3$ \\
\hline
\end{tabular}

$100 \mathrm{~km} \mathrm{~s}^{-1}$ (see Table E.1) and decreases in group G and C $\left(20-50 \mathrm{~km} \mathrm{~s}^{-1}\right)$.

HH $53 \mathrm{~A}, \mathrm{~B}$, and $\mathrm{C}$ have similar inclinations $\left(83^{\circ} \pm 2^{\circ}\right)$ and spatial velocities (around $110 \mathrm{~km} \mathrm{~s}^{-1}$ ). HH $53 \mathrm{C} 1$ is superimposed on the second flow and is moving accordingly with the HH 52 streamer, with an inclination angle of $\sim 49^{\circ} \pm 9^{\circ}$,
Table E.1. Inclination and spatial velocities of individual knots of HH 52, 53, and 54 inferred from the kinematical analysis.

\begin{tabular}{ccc}
\hline \hline knot ID & $i\left(^{\circ}\right)$ & $v_{\text {tot }}\left(\mathrm{km} \mathrm{s}^{-1}\right)$ \\
\hline HH 52 A1 & $58 \pm 4$ & $93 \pm 6$ \\
HH 52 A3-A4 & $57 \pm 2$ & $83 \pm 6$ \\
HH 52 A & $62 \pm 4$ & $106 \pm 14$ \\
HH 52 B & $63 \pm 3$ & $88 \pm 22$ \\
HH 52 D2-D3 & $67 \pm 4$ & $112 \pm 20$ \\
HH 52 D4 & $61 \pm 3$ & $83 \pm 11$ \\
HH 53 A & $81 \pm 1$ & $111 \pm 11$ \\
HH 53 B & $85 \pm 1$ & $113 \pm 10$ \\
HH 53 C & $83 \pm 1$ & $105 \pm 8$ \\
HH 53 C1 & $49 \pm 9$ & $76 \pm 21$ \\
HH 53 E1 & $57 \pm 3$ & $85 \pm 9$ \\
HH 53 F2 & $63 \pm 3$ & $100 \pm 15$ \\
HH 54 C2 & $61 \pm 3$ & $51 \pm 11$ \\
HH 54 C3 & $52 \pm 8$ & $24 \pm 9$ \\
HH 54 G-G0 & $55 \pm 8$ & $60-95 \pm 20$ \\
HH 54 G1-G3 & $53 \pm 8$ & $70 \pm 20$ \\
HH 54 X1A & $66 \pm 6$ & $77 \pm 21$ \\
HH 54 X1D-X4C & $64 \pm 7$ & $65 \pm 24$ \\
HH 54 X3 & $67 \pm 10$ & $50 \pm 15$ \\
HH 54 X4A-X4B & $68 \pm 4$ & $124 \pm 23$ \\
\hline
\end{tabular}

considering the low velocity component measured in HH 53 C-C1 spectrum (see Table D.2).

In the HH54 streamer we measure an average inclination angle of $67 \pm 3^{\circ}$, but the errors of the single data points are too large to derive variations of inclination along the wiggling jet. 